\title{
Disordered Icosahedral Boron-Rich Solids - A Theoretical Study of Thermodynamic Stability and Properties
}

\author{
Annop Ektarawong
}


The cover page illustrates the crystal structure of disordered boron carbide $\left(\mathrm{B}_{4} \mathrm{C}\right)$, projected on the $(100)_{\text {r }}$ crystal plane in rhombohedral symmetry.

The cover image is visualized by the VESTA package.

Red and white spheres represent boron and carbon atoms, respectively.

During the course of research underlying this thesis, Annop Ektarawong was enrolled in Agora Materiae, a multidisciplinary doctoral program at Linköping University, Sweden.

(C) Annop Ektarawong ISBN 978-91-7685-544-7

ISSN 0345-7524

Printed by LiU-Tryck 2017 
This thesis is a theoretical study of configurational disorder in icosahedral boronrich solids, in particular boron carbide, including also the development of a methodological framework for treating configurational disorder in such materials, namely superatom-special quasirandom structure (SA-SQS). In terms of its practical implementations, the SA-SQS method is demonstrated to be capable of efficiently modeling configurational disorder in icosahedral boron-rich solids, whiles the thermodynamic stability as well as the properties of the configurationally disordered icosahedral boron-rich solids, modeled from the SA-SQS method, can be directly investigated, using the density functional theory (DFT).

In case of boron carbide, especially $\mathrm{B}_{4} \mathrm{C}$ and $\mathrm{B}_{13} \mathrm{C}_{2}$ compositions, the SA-SQS method is used for modeling configurational disorder, arising from a high concentration of low-energy $\mathrm{B} / \mathrm{C}$ substitutional defects. The results, obtained from the DFT-based calculations, demonstrate that configurational disorder of $\mathrm{B}$ and $\mathrm{C}$ atoms in boron carbide is not only thermodynamically favored at high temperature, but it also plays an important role in altering the properties of boron carbide - for example, restoration of higher rhombohedral symmetry of $\mathrm{B}_{4} \mathrm{C}$, a metal-tononmetal transition and a drastic increase in the elastic moduli of $\mathrm{B}_{13} \mathrm{C}_{2}$. The configurational disorder can also explain large discrepancies, regarding the properties of boron carbide, between experiments and previous theoretical calculations, having been a long standing controversial issue in the field of icosahedral boronrich solids, as the calculated properties of the disordered boron carbides are found to be in qualitatively good agreement with those, observed in experiments. In order to investigate the configurational evolution of $\mathrm{B}_{4} \mathrm{C}$ as a function of temperature, beyond the SA-SQS level, a brute-force cluster-expansion method in combination with Monte Carlo simulations is implemented. The results demonstrate that configurational disorder in $\mathrm{B}_{4} \mathrm{C}$ indeed essentially takes place within the icosahedra in a way that justifies the focus on low-energy defect patterns of the superatom picture.

The investigation of the thermodynamic stability of icosahedral carbon-rich 
boron carbides beyond the believed solubility limit of carbon (20 at.\% C) demonstrates that, apart from $\mathrm{B}_{4} \mathrm{C}$ generally addressed in the literature, $\mathrm{B}_{2.5} \mathrm{C}$ represented by $\mathrm{B}_{10} \mathrm{C}_{2}^{p}(\mathrm{CC})$ is predicted to be thermodynamically stable with respect to $\mathrm{B}_{4} \mathrm{C}$ as well as pure boron and carbon under high pressure, ranging between 40 and $67 \mathrm{GPa}$, and also at elevated temperature. $\mathrm{B}_{2.5} \mathrm{C}$ is expected to be metastable at ambient pressure, as indicated by its dynamical and mechanical stabilities at 0 GPa. A possible synthesis route of $\mathrm{B}_{2.5} \mathrm{C}$ and a fingerprint for its characterization from the simulations of $\mathrm{x}$-ray powder diffraction pattern are suggested.

Besides modeling configurational disorder in boron carbide, the SA-SQS method also opens up for theoretical studies of new alloys between different icosahedral boron-rich solids - for example, $\left(\mathrm{B}_{6} \mathrm{O}\right)_{1-x}\left(\mathrm{~B}_{13} \mathrm{C}_{2}\right)_{x}$ and $\mathrm{B}_{12}\left(\mathrm{As}_{1-x} \mathrm{P}_{x}\right)_{2}$. As for the pseudo-binary $\left(\mathrm{B}_{6} \mathrm{O}\right)_{1-x}\left(\mathrm{~B}_{13} \mathrm{C}_{2}\right)_{x}$ alloy, it is predicted to display a miscibility gap resulting in $\mathrm{B}_{6} \mathrm{O}$-rich and either ordered or disordered $\mathrm{B}_{13} \mathrm{C}_{2}$-rich domains for intermediate global compositions at all temperatures up to melting points of the materials. However, some intermixing of $\mathrm{B}_{6} \mathrm{O}$ and $\mathrm{B}_{13} \mathrm{C}_{2}$ to form solid solutions is also predicted at high temperature. A noticeable mutual solubility of icosahedral $\mathrm{B}_{12} \mathrm{As}_{2}$ and $\mathrm{B}_{12} \mathrm{P}_{2}$ in each other to form $\mathrm{B}_{12}\left(\mathrm{As}_{1-x} \mathrm{P}_{x}\right)_{2}$ disordered alloy is predicted even at room temperature, and a complete closure of a pseudo-binary miscibility gap is achieved at around $900 \mathrm{~K}$.

Apart from $\mathrm{B}_{12}\left(\mathrm{As}_{1-x} \mathrm{P}_{x}\right)_{2}$, the thermodynamic stability of other compounds and alloys in the ternary B-As-P system is also investigated. For the binary B-As system, zinc-blende BAs is found to be thermodynamically unstable with respect to icosahedral $\mathrm{B}_{12} \mathrm{As}_{2}$ and gray arsenic at $0 \mathrm{~K}$ and increasingly so at higher temperature, indicating that BAs may merely exist as a metastable phase. This is in contrast to the binary $\mathrm{B}-\mathrm{P}$ system, in which zinc-blende $\mathrm{BP}$ and icosahedral $\mathrm{B}_{12} \mathrm{P}_{2}$ are both predicted to be stable. Owing to the instability of BAs with respect to $\mathrm{B}_{12} \mathrm{As}_{2}$ and gray arsenic, only a tiny amount of BAs is predicted to be able to dissolve in $\mathrm{BP}$ to form $\mathrm{BAs}_{1-x} \mathrm{P}_{x}$ disordered alloy at elevated temperature. For example, less than 5\% BAs can dissolve in BP at $1000 \mathrm{~K}$. As for the binary As-P system, $\mathrm{As}_{1-x} \mathrm{P}_{x}$ disordered alloys are predicted at elevated temperature - for example, a disordered solid solution of up to $\sim 75 \%$ As in black phosphorus as well as a small solubility of $\sim 1 \% \mathrm{P}$ in gray arsenic at $750 \mathrm{~K}$, together with the presence of miscibility gaps.

The thermodynamic stability of three different compositions of $\alpha$-rhombohedral boron-like boron subnitride, having been proposed so far in the literature, is investigated. Those are, $\mathrm{B}_{6} \mathrm{~N}, \mathrm{~B}_{13} \mathrm{~N}_{2}$, and $\mathrm{B}_{38} \mathrm{~N}_{6}$, represented respectively by $\mathrm{B}_{12}(\mathrm{~N}-\mathrm{N})$, $\mathrm{B}_{12}(\mathrm{NBN})$, and $\left[\mathrm{B}_{12}(\mathrm{~N}-\mathrm{N})\right]_{0.33}\left[\mathrm{~B}_{12}(\mathrm{NBN})\right]_{0.67}$. It is found that, out of these subnitrides, only $\mathrm{B}_{38} \mathrm{~N}_{6}$ is thermodynamically stable from $0 \mathrm{GPa}$ up to $\sim 7.5 \mathrm{GPa}$, depending on the temperature, and is thus concluded as a stable composition of $\alpha$-rhombohedral boron-like boron subnitride. 


\section{Populärvetenskaplig sammanfattning}

I denna avhandling genomförs teoretiska undersökningar av en specifik klass av bor-rika material. I denna materialklass domineras kristallstrukturen av ikosaedrer formade av 12 atomer, mestadels bor, men i vissa fall med inlösningar av andra grundämnen, särskilt kol. Utöver ikosaedrer förekommer även andra geometriska motiv av atomer vilket ytterligare komplicerar strukturen. Av detta skäll är det är synnerligen utmanande att förstå exakt hur atomerna är ordnade eller ifall det förekommer någon form av oordning mellan dem. Samtidigt så är en detaljerad kunskap om atomordningen nödvändig för att kunna beräkna termodynamisk stabilitet liksom materialens elektriska, vibrationella och elastiska egenskaper.

En metod för att bryta ner det totala strukturproblemet i hanterbara storheter, superatomer, exempelvis en $\mathrm{B}_{11} \mathrm{C}$ ikosaeder med specifik placering av kolatomen, och oordning dem emellan, föreslås i detta arbete. Metoden utvärderas i ett ramverk av första-princip-beräkningar. Kvantmekaniska täthetsfunktionalberäkningar samt gitterdynamik-metoder för utvärdering av elektroniska och vibrationella effekter används genomgående i avhandlingen

Metoden eller synsättet för att förstå oordning i dessa material, Superatomsspeciella quasislumpmässiga strukturer (SA-SQS), visar sig kunna ge kvantitativa förutsägelser om flera egenskaper hos materialen, och används för att förutspå termodynamisk fasstabilitet som en funktion av temperatur och tryck för kända och ännu icke tillverkade legeringar baserade på bor.

Ett specifikt fokus är på borkarbid, en förening mellan bor och kol ofta med sammansättningen $\mathrm{B}_{4} \mathrm{C}$ där kolatomernas exakta placering i kristallen vållat betydande kontroverser mellan tidigare vetenskapliga studier. Avhandlingens resultat visar att bland annat den observerade kristallsymmetrin hos $\mathrm{B}_{4} \mathrm{C}$ kan förstår med hjälp av oordnade kolatomer. Att borkarbid uppmäts till att vara halvledare och inte metalliskt ledande även vid sammansättningen $\mathrm{B}_{13} \mathrm{C}_{2}$ kan även det förklaras med den mest sannolika typen av atomisk oordning, och det kan även de elastiska egenskaperna hos $\mathrm{B}_{13} \mathrm{C}_{2}$.

Vidare undersöks möjlig stabilitet för extra kol-rika borkarbidfaser som en 
funktion av temperatur och tryck. Resultatet förutspår att en ännu icke observerad fas $\mathrm{B}_{2.5} \mathrm{C}$ bör kunna observeras vid högt tryck, oavsett temperatur.

Legeringar mellan borkarbid och borsuboxid undersöks och möjligheten för en viss fast lösning på superatoms-nivå mellan dessa likartade faser förutspås och deras elastiska egenskaper utvärderas. Bor-Arsenik-Fosfor systemet utvärderas och BAs-fasen visar sig vara termodynamisk instabil med avseende på $\mathrm{B}_{12} \mathrm{As}_{2}$ och $\mathrm{As}$ tillskillnad från BP som är stabil. Stabila ternära $\mathrm{B}_{12}(\mathrm{As}, \mathrm{P})_{2}$-legeringar förutspås kunna existera vid temperaturer från rumstemperatur och uppåt.

Borsubnitrid har diskuterats av tidigare forskare men det är kontroversiellt vilken eller vilka som är ämnets stabila sammansättningar. I denna avhandling undersöks den termodynamiska stabiliteten hos $\mathrm{B}_{6} \mathrm{~N}, \mathrm{~B}_{13} \mathrm{~N}_{2}$, och $\mathrm{B}_{38} \mathrm{~N}_{6}$, som alla föreslagits tidigare. Enbart $\mathrm{B}_{38} \mathrm{~N}_{6}$, som strukturellt kan ses som en legering mellan de två andra sammansättningarna på superatom-nivå visar sig vara stabil och dess elektriska, vibrationella, och elastiska egenskaper beräknas och redovisas. 
This thesis is a summary of research, as a Ph.D. student in the Thin Film Physics Division at Linköping University from January 2013 to June 2017. The methodology chapters of this doctoral thesis are based on my previous licentiate thesis in November 2015, First-principles study of configurational disorder in icosahedral boron-rich solids, Thesis No. 1731.

The work has been focused on theoretical investigations of influences of configurational disorder on the stabilities and properties of icosahedral boron-rich solids, and mixing thermodynamics of different icosahedral boron-rich solids. Those are, boron carbides, boron suboxide, boron subpnictides, and boron subnitrides. The results, carried out during the study period, are presented either in the form of scientific papers, published in peer-reviewed journals, or as manuscripts submitted or in final preparation for submission to peer-reviewed journals.

Financial support for this thesis work is mainly provided by the Swedish Research Council (VR). The theoretical calculations are carried out using supercomputer resources, provided by the Swedish National Infrastructure for Computing (SNIC) performed at the National Supercomputer Centre (NSC) and the Center for High Performance Computing (PDC). 



\section{Acknowledgements}

First of all, I would like to express my greatest gratitude to my supervisor Docent Björn Alling for providing me a ton of knowledge and good scientific attitudes. You have been taking a good care of me, since I got accepted as your Ph.D. student. I have learned many valuable things, and gained a lot of invaluable experience in researching from you.

I would also like to thank my assistant supervisors, Prof. Sergei Simak and Prof. Lars Hultman, for your kind assistances that are indeed essential to complete this thesis.

A special thanks goes to everyone in the Thin Film Physics and Theoretical Physics groups, and Agora Materiae at IFM, Linköping University for lots of help and ideas. Your contributions are also parts to get this thesis successful.

I am very grateful for Dr. Yuttapoom Puttisong and his wife Promporn Wangwacharakul for uncountable help with everything. You two have always provided me good advices, and taught me a lot of things.

Thanks to Araya Eamrurksiri and Varaporn Ongart for being a good friend.

My most greatest thanks goes to every member in my family, who have always been supporting me with everything they have gotten, since I was born. I would not have been able to come this far without you.

I would finally like to thank Nutpapas Khanawatpitikhun for always standing by my side and never being tired of supporting me. You are the best!

Annop Ektarawong

Linköping, June 2017 

1 Icosahedral boron-rich solids $\quad 1$

1.1 Introduction . . . . . . . . . . . . . . . . . . . . . 1

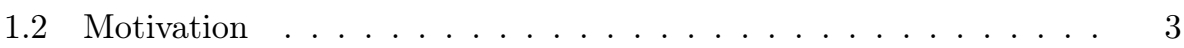

1.3 Boron carbide . . . . . . . . . . . . . . . . . . 4

1.4 Boron suboxide . . . . . . . . . . . . . . . . 7

1.5 Boron subarsenide and subphosphide . . . . . . . . . . . . . 8

1.6 Boron subnitride . . . . . . . . . . . . . . . . . 9

2 Density functional theory 11

2.1 Theoretical background ................. 11

2.2 The Hohenberg-Kohn theorems . . . . . . . . . . . . . . . . . . . . 13

2.3 Kohn-Sham equations . . . . . . . . . . . . . . . 14

2.4 Exchange-correlation functional . . . . . . . . . . . . . . . . 15

2.5 Plane-wave basis set . . . . . . . . . . . . . . . . . . . . . . . . . . . . . . . 17

2.6 Solving the Kohn-Sham equations . . . . . . . . . . . . . . 18

3 Lattice dynamics $\quad 19$

3.1 Introduction . . . . . . . . . . . . . . . . . . . . . 19

3.2 Harmonic approximation . . . . . . . . . . . . . . 20

3.3 Quasi-harmonic approximation ............. 23

4 Configurational disorder in crystalline solids $\quad 25$

4.1 Modeling of configurational disorder . . . . . . . . . . . . . 25

4.2 Connolly-Williams cluster expansion method . . . . . . . . . . 27

4.3 Special quasirandom structure method . . . . . . . . . . . . 27

4.4 Superatom-special quasirandom structure . . . . . . . . . . . . 28 
5 Thermodynamic stability $\quad 35$

5.1 Convex hull and formation energy . . . . . . . . . . . . . 37

5.2 Estimation of an order-disorder transition temperature in an alloy at a given composition . . . . . . . . . . . . . . . 44 40

5.3 Mixing thermodynamics of alloys . . . . . . . . . . . . . . . . 42

5.4 Thermodynamic stability of ternary compounds and alloys: Linear optimization problem . . . . . . . . . . . . . 44

6 Modeling elastic properties $\quad 47$

7 Conclusions $\quad \mathbf{5 3}$

7.1 Methodological development . . . . . . . . . . . . 53

7.2 Materials stabilities and properties . . . . . . . . . . . . 54

8 Outlook $\quad 59$

$\begin{array}{ll}\text { Bibliography } & 61\end{array}$

$\begin{array}{ll}\text { List of included publications } & 73\end{array}$

$\begin{array}{ll}\text { Related, not included publication } & 75\end{array}$

$\begin{array}{lll}9 & \text { Summary of included publications } & 77\end{array}$

$\begin{array}{lr}\text { Paper I } & 81\end{array}$

$\begin{array}{ll}\text { Paper II } & 95\end{array}$

$\begin{array}{ll}\text { Paper III } & 109\end{array}$

$\begin{array}{lr}\text { Paper IV } & 123\end{array}$

$\begin{array}{ll}\text { Paper V } & 133\end{array}$

$\begin{array}{lr}\text { Paper VI } & 149\end{array}$

$\begin{array}{ll}\text { Paper VII } & 163\end{array}$ 
CHAPTER 1

\section{Icosahedral boron-rich solids}

\subsection{Introduction}

Over the last few decades, icosahedral boron-rich solid have been of interest across a wide range of research areas in materials science. The solids primarily comprise of 12-atom icosahedral clusters, made mainly of boron atoms. The simplest form of icosahedral boron-rich solids is elementary $\alpha$-rhombohedral boron $\left(\mathrm{B}_{12}\right)$, whose crystal structure is represented by $\mathrm{B}_{12}$ icosahedral clusters placed at vertices of a rhombohedral unitcell with the space group of $R \overline{3} m[1,2]$, as shown by Fig.1.1(a). As a consequence of the translational symmetry of the crystal, the $\mathrm{B}_{12}$ clusters lose their fivefold rotational axes and thus consist of two crystallographic sites, namely polar and equatorial. As for $\alpha$-rhombohedral boron, each $\mathrm{B}_{12}$ cluster links to six neighboring $\mathrm{B}_{12}$ clusters through atoms residing in the polar sites by forming direct inter-icosahedral bonds. Other icosahedral boron-rich solids, e.g., boron carbide $\left(\mathrm{B}_{4} \mathrm{C}\right)$, boron suboxide $\left(\mathrm{B}_{6} \mathrm{O}\right)$, and boron subpnictides $\left(\mathrm{B}_{12} \mathrm{As}_{2}\right.$ and $\left.\mathrm{B}_{12} \mathrm{P}_{2}\right)$, are derivatives of $\alpha$-rhombohedral boron. In the case of boron carbide [2-5], triatomic linear chains composing either of boron or carbon occupy the interstices between the icosahedral clusters and align themselves along the [111] direction of the rhombohedron, as illustrated in Fig.1.1(b). Each chain-end atom bridges the three neighboring icosahedra by forming chemical bonds with the equatorial atoms. Rather than the triatomic chains, diatomic chains of arsenic and phosphorus are formed for $\mathrm{B}_{12} \mathrm{As}_{2}$, and $\mathrm{B}_{12} \mathrm{P}_{2}$, respectively [1, 6], see Fig.1.1(c). As for $\mathrm{B}_{6} \mathrm{O}$, two isolated oxygen atoms occupy the interstices between the icosahedra, being too small to form the diatomic chains [7, 8], as can be seen from Fig.1.1(d).

Apart from the examples given above, there exist other forms of icosahedral boron-rich solids, exhibiting even more complex crystal structures, e.g., elementary $\beta$-rhombohedral boron $\left(\mathrm{B}_{105}\right)$, elementary $\gamma$-boron $\left(\mathrm{B}_{28}\right)$, and silicon hexaboride 
$\left(\mathrm{SiB}_{6}\right)$. Although the crystal symmetry and space group of $\beta$-rhombohedral boron are identical to those of $\alpha$-rhombohedral boron, its crystal structure is far more complicated in which, apart from regular $\mathrm{B}_{12}$ icosahedral clusters, it can consist of larger clusters of boron atoms $\left(\mathrm{B}_{84}\right)$, deltahedra $\left(\mathrm{B}_{10}\right)$, and individual boron atoms $[9,10]$. Due to its highly complex atomic structure, several structural models of $\beta$-boron have been proposed in the literature [11-13]. An issue, regarding both the number of boron atoms and the structure of boron clusters in the unitcell of $\beta$-rhombohedral boron, has however been inconclusive and still being a debate among the community. On the other hand, $\gamma$-boron, a high-pressure phase of elementary boron, consists of two $\mathrm{B}_{12}$ icosahedra and two $\mathrm{B}_{2}$ dumbbells with a $\mathrm{NaCl}$-type of arrangement in an orthorhombic unitcell [14, 15]. $\mathrm{SiB}_{6}$ has also a rather complex atomic configuration. Within an orthorhombic unitcell, it contains not only regular icosahedra but also icosihexahedra, and isolated boron and silicon atoms [16].

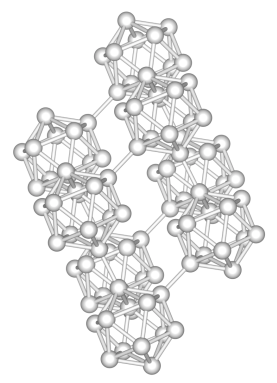

(a)

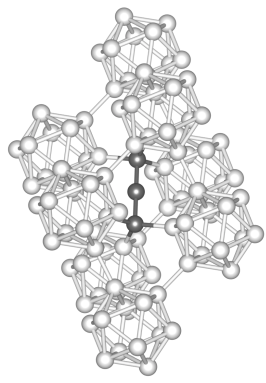

(b)

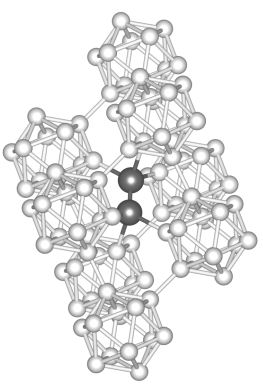

(c)

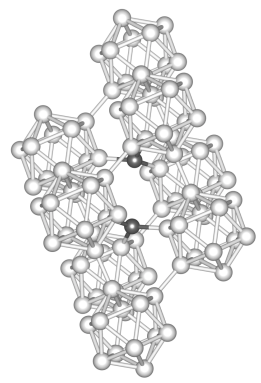

(d)

Figure 1.1. Crystal structures of (a) $\alpha$-boron, (b) boron carbide, (c) boron subpnictides, and (d) boron suboxide. White and grey spheres represent atoms residing in the 12-atom icosahedra and in the interstices between the icosahedra, respectively.

Chemical bonds between atoms, residing in the icosahedral clusters, are unique and do not obey the regular bonding criteria, established for describing two-center two-electron bonds. For example, in the case of $\alpha$-rhombohedral boron, each boron atoms, forming an icosahedron, bonds to five neighboring boron atoms. Based on the regular bonding criteria, this is not possible, since at least five electrons are required from each boron atom to fulfil the criteria, while each boron atom can provide at most three electrons $\left(2 \mathrm{~s}^{2}, 2 \mathrm{p}^{1}\right)$ for the chemical bond formation. Due to their electron deficiency, the boron atoms form three-center two-electron bonds, in which each triangular face of the icosahedron comprising of three boron atoms are sharing merely two electrons, and the electron density is peaked around the center of the triangle [17]. Demonstrated by Longuet-Higgins and Roberts [18], twenty-six electrons are required to complete the thirteen intra-icosahedral bonding orbitals. As a result, twenty-six out of thirty-six electrons of the twelve boron atoms are used in forming the $\mathrm{B}_{12}$ icosahedron. Another six electrons are used 
to form the two-center inter-icosahedral bonds between the polar atoms with the six neighboring icosahedra. The rest four electrons are contributed to the formation of the three-center inter-icosahedral bonds with the neighboring icosahedra through the equatorial atoms. Since all of the available electrons are used up to fill the bonding, $\alpha$-boron is a semiconductor [17]. Similar to $\alpha$-boron, the concept of three-center two-electron bond can also be applied to describe the electronic character of the other $\alpha$-rhombohedral boron-like solids - for example, $\mathrm{B}_{12} \mathrm{P}_{2}$, $\mathrm{B}_{6} \mathrm{O}$ and $\mathrm{B}_{4} \mathrm{C}[17-19]$.

Owing to their unusual crystal structures and bonding types, together with the light atomic mass of boron, icosahedral boron-rich solids display several outstanding properties [6, 17], such as high chemical stability, high hardness, high melting point, low density, and low wear coefficient. Such properties thus make the materials promising for a wide range of technological applications, for instance light weight armor, wear-resistant devices, cutting tools [20-25], candidates for high temperature electronic $[6,26,27]$ and thermoelectric devices $[1,6,28-30]$, etc. In addition, due to a large thermal neutron absorption cross section of the isotope ${ }^{10} \mathrm{~B}$, icosahedral boron-rich solids can be used as neutron absorbing materials in nuclear reactors $[6,20,31,32]$. Note that naturally boron contains approximately 20 at. $\%$ of ${ }^{10} \mathrm{~B}$. Recently, boron-rich solids, especially $\mathrm{B}_{4} \mathrm{C}$, have been realized as solid state thermal neutron detectors [33-35].

\subsection{Motivation}

Although icosahedral boron-rich solids have been intensively studied over many decades both experimentally and theoretically, resulting in valuable knowledge and increased understanding of the materials, there exist large discrepancies between them, thus giving rise to uncertainties in the atomic configuration, thermodynamic stability and properties of these boron-rich solids. The central issue of these controversies may be attributed to configurational disorder of different atomic species on the lattice sites. A distinct example is boron carbide, which is stable as a singlephase solid solution over a wide range of compositions $[20,36]$. Investigation of configurational disorder is, in this case, experimentally complicated not only due to the similar atomic form factors of boron and carbon atoms [37], but due also to the difficulties to probe the material in the equilibrium condition, resulting from limited atomic mobility at low temperature in ceramic materials. On the other hand, implementation of theoretical frameworks, such as the cluster expansion [38-40] and the special quasirandom structure (SQS) methods [41] to address the problem of configurational disorder in such materials is not trivial, owing to the complexity of the icosahedral structure composing of different sublattices as well as the unusual chemistry, as compared to standard metal-alloys, with the possibility of a very strong preference to avoid specific types of bonds at certain crystallographic sites - for instance, inter-icosahedral C-C bonds in the case of boron carbide (Paper I, II, and III).

As a consequence, investigation of configurational disorder in icosahedral boronrich solids has remained a challenge also for the alloy theoreticians. This thus gives 
rise to a number of open questions. For example, which types of configurational disorder dominate in icosahedral boron-rich solids, especially boron carbide? What are the consequences of configurational disorder in terms of properties? What are the stable compositions for a particular boron-rich solid and are they influenced by configurational disorder? Also, theoretical method development is needed in order to make first-principles based investigations of configurationally disordered icosahedral boron-rich solids practically feasible. Such a methodology would also open up for theoretical studies of new alloys between different boron-rich solids. In addition to the configurational disorder, lattice vibrations are also expected to significantly influence the phase stability in these materials, comprising of light elements, such as boron, and carbon, at elevated temperature [42]. Such contributions have, however, been largely neglected in the previous theoretical studies of icosahedral boron-rich solids.

This thesis aims at addressing these general questions, developing a methodological framework for treating configurational disorder in this class of materials accurately and efficiently, and applying it in first-principles studies of different icosahedral boron-rich solids. Special attention is paid to those, whose structures are derivatives of $\alpha$-rhombohedral boron, i.e., boron carbide, boron suboxide and boron subpnictides. Brief descriptions of the considered boron-rich solids, including their specific open questions and uncertainties, are given in the sections below. In the following chapters, the methodological basis of this thesis work is then described, followed by a short summary of the results and an outlook for future work. The detailed results of the investigations are, on the one hand, presented either in the form of scientific papers, published in peer-reviewed journals, or as manuscripts submitted or in final preparation for submission to peer-reviewed journals.

\subsection{Boron carbide}

Boron carbide was discovered in 1858 [20]. Despite the fact that the material has been known for more than 150 years, several issues about its properties remain ambiguous. Confirmed by several independent experiments [20, 36], boron carbide is stable as a single-phase solid solution over a broad composition range. Even though a lot of B-C phase diagrams has been suggested from time to time in the literature [20, 43-46], they are still being argued among the community. It is however generally accepted that the single-phase region of boron carbide extends from 8 to 20 at.\% $\mathrm{C}$ approximately. The proper stoichiometric formula of boron carbide is thus preferably given by $\mathrm{B}_{1-x} \mathrm{C}_{x}$, where $0.08 \lesssim x \lesssim 0.2$ corresponding to designations of $\mathrm{B}_{11.5} \mathrm{C}$ to $\mathrm{B}_{4} \mathrm{C}$. Revealed by $\mathrm{x}$-ray and neutron diffraction measurements [2-5], a fundamental crystalline structure of boron carbide can be seen as a modification of $\alpha$-rhombohedral boron ( $R \overline{3} \mathrm{~m}$ space group), whose basic structural unit can be represented by a 12-atom icosahedron connecting to a triatomic chain, as displayed by Fig.1.1(b). However, precisely determining the atomic positions of carbon atoms in boron carbide at any specific carbon content is extremely difficult, owning to the similarities both of atomic form factor for x-ray diffraction [37] and of nuclear scattering cross-sections $\left({ }^{11} \mathrm{~B}\right.$ and $\left.{ }^{12} \mathrm{C}\right)$ for neutron diffraction $[5,47]$ 
between boron and carbon atoms. A lot of effort has so far been done not only to determine how boron and carbon atoms are residing on the lattice sites, but also to understand how the atomic configuration of boron and carbon atoms changes with the carbon content, varying within the single-phase region, and how such a change influences the materials properties. Up to now, several theoretical models of boron carbide have been suggested in the literature, mainly focusing on $\mathrm{B}_{12} \mathrm{C}_{3}$ $\left(\mathrm{B}_{4} \mathrm{C}\right)$ and $\mathrm{B}_{13} \mathrm{C}_{2}$ with 20 and 13.3 at.\% $\mathrm{C}$, respectively.

\section{Theoretical models of $\mathrm{B}_{4} \mathrm{C}$ and $\mathrm{B}_{13} \mathrm{C}_{2}$}

A theoretical model of $\mathrm{B}_{4} \mathrm{C}$ was firstly suggested with an aim to preserve the $R \overline{3} \mathrm{~m}$ space group, where the basic structural unit was given by a $\mathrm{B}_{12}$ icosahedron and a triatomic chain purely made of carbon, denoted by $\mathrm{B}_{12}(\mathrm{CCC})$ [3]. However, it was shown later by nuclear magnetic resonance (NMR) studies [48-50], x-ray [2, 51] and neutron [52] diffractions that rather than $\mathrm{B}_{12}(\mathrm{CCC})$, the basic unit of $\mathrm{B}_{4} \mathrm{C}$ should be represented by a $\mathrm{B}_{11} \mathrm{C}$ icosahedron as well as a (CBC) chain, in which one of the icosahedral boron atoms is swapped with the middle carbon atom in the chain. Further clarified by first-principles calculations [53-57], $\mathrm{B}_{11} \mathrm{C}^{p}(\mathrm{CBC})$ is found to be the most energetically favorable basic unit over the other units, e.g., $\mathrm{B}_{11} \mathrm{C}^{e}(\mathrm{CBC}), \mathrm{B}_{12}(\mathrm{CCC})$, and $\mathrm{B}_{11} \mathrm{C}^{p}(\mathrm{CCB})$, where the superscript $p$ and $e$ denote the polar and equatorial sites, respectively. However, swapping the middlechain carbon atom with the icosahedral boron atom to obtain the more favorable model of $\mathrm{B}_{11} \mathrm{C}^{p}(\mathrm{CBC})$ introduces a distortion, reducing the crystal symmetry of boron carbide to base-centered monoclinic with the $C m$ space group $[56,57]$. This gives rise to a discrepancy in the crystal symmetry of boron carbide between experiments and theoretical calculations. Furthermore, a comparison between the theoretical and experimental NMR spectra of $\mathrm{B}_{4} \mathrm{C}$ [58] suggests also the presence of $\mathrm{B}_{12}(\mathrm{CBC})$ and $\mathrm{B}_{10} \mathrm{C}_{2}^{p}(\mathrm{CBC})$ units. Thus the assumption, that only the presumed $\mathrm{B}_{11} \mathrm{C}^{p}(\mathrm{CBC})$ unit represents the whole structure of $\mathrm{B}_{4} \mathrm{C}$, does not seem to be true.

If the carbon concentration of boron carbide is decreasing from 20 at. $\% \mathrm{C}$ with the presumed $\mathrm{B}_{11} \mathrm{C}(\mathrm{CBC})$ toward 13.3 at.\% $\mathrm{C}$, that is the $\mathrm{B}_{13} \mathrm{C}_{2}$ composition, the substitution of boron for carbon atoms can take place either within the icosahedron or within the chain. Substitution of boron for carbon atom within the icosahedron results in the $\mathrm{B}_{12}(\mathrm{CBC})$ unit, while the $\mathrm{B}_{11} \mathrm{C}(\mathrm{BBC})$ unit will be achieved if the substitution takes place in the chains. Judging by the total energies, obtained from first-principles calculations [59-61], $\mathrm{B}_{12}(\mathrm{CBC})$ exhibits considerably lower energy as compared to $\mathrm{B}_{11} \mathrm{C}(\mathrm{BBC})$, and thus often being discussed in the literature. The latter model is, however, consistent with the analyses of structural data from x-ray diffraction [62] and Raman spectra [63-65] of boron carbide at different at.\% $\mathrm{C}$ in the single-phase region.

\section{Electronic properties calculations of $\mathrm{B}_{4} \mathrm{C}$ and $\mathrm{B}_{13} \mathrm{C}_{2}$}

Apart from the argument, regarding the representation of the structural units, there is also a discrepancy in the electronic character between theory and experiment. According to the reports, found in the literature [66, 67], boron carbide is 
experimentally found to be a semiconductor throughout the single-phase region, whose electronic band gap falls into the range between 0.48 and $2.5 \mathrm{eV}$ [68-71], depending on the composition and the crystalline quality of boron carbide. Based on the explanation of Longuet-Higgins and Roberts [18], forty-eight electrons are required in order to complete the valence band of boron carbide, and gives rise to a semiconducting character. This condition is, however, satisfied only for the exact $\mathrm{B}_{4} \mathrm{C}$ composition, while, e.g., the $\mathrm{B}_{13} \mathrm{C}_{2}$ composition should, in principle, have been metallic due to its electron deficiency as the number of electrons decreases from the substitution of boron for carbon atoms. Such explanation is in line with the results, obtained from the theoretical calculations of electronic properties, demonstrating that $\mathrm{B}_{12}(\mathrm{CBC})$ is metallic $[53,59,72]$. Even though $\mathrm{B}_{4} \mathrm{C}$ is predicted to be a semiconductor, in qualitative agreement with the experimental findings, the calculated electronic band gap of the most favorable $\mathrm{B}_{11} \mathrm{C}^{p}(\mathrm{CBC})$ is overestimated, ranging from 2.6 up to $3.0 \mathrm{eV}[9,73,74]$. It is worth noting that the electronic band gaps, almost without exception, are typically underestimated in standard density functional theory calculations due to the approximation of the exchange-correlation functionals (see Chapter 2.4). Note also that a smaller band gap of about $1.6 \mathrm{eV}$ can be achieved from the high-energy $\mathrm{B}_{12}(\mathrm{CCC})$ unit due to the appearance of the mid gap states [75].

\section{Configurational disorder in boron carbide}

Convinced by the large solubility range of carbon, it has been suggested in the literature $[56,76,77]$ that the large discrepancies in the properties of boron carbide between experiments and theoretical calculations could be arising from configurational disorder, induced by $\mathrm{B} / \mathrm{C}$ substitutional defects. However, due to the similar characteristics between boron and carbon atoms, experimentally quantifying the presence of configurational disorder in boron carbide is a formidable task. Owing to the complexity of the icosahedral network, numerous kinds of $\mathrm{B} / \mathrm{C}$ defects are imaginable. As a consequence, theoretical modeling of configurational disorder in boron carbide using the standard approaches, such as the cluster expansion [38-40] and the SQS [41] methods, is not trivial. Implementation of the coherent potential approximation (CPA) [78], on the other hand, is not suitable to treat the configurational disorder in this case, as it cannot account for lattice relaxations. In this thesis, a newly developed method, superatom-special quasirandom structure (SASQS), is employed for modeling configurational disorder in boron carbide, arising from a high concentration of low-energy $\mathrm{B} / \mathrm{C}$ substitutional defects. Impacts of the configurational disorder, arising from a high concentration of low-energy $\mathrm{B} / \mathrm{C}$ substitutional defects, on the thermodynamic stability as well as the properties of boron carbide are then investigated, using first-principles calculations. Results from the studies are presented and discussed in Paper I, II, III, and IV, in which $\mathrm{B}_{4} \mathrm{C}$ and $\mathrm{B}_{13} \mathrm{C}_{2}$ are the two stoichiometries, mainly considered. 


\section{Carbon-rich boron carbide beyond $\mathrm{B}_{4} \mathrm{C}$}

It is widely accepted, as indicated by several theoretical studies [56-58, 60, 61, 73], that near the solubility limit of carbon $(\sim 20$ at.\% C), the basic structural units of boron carbide are dominated by $\mathrm{B}_{11} \mathrm{C}^{p}(\mathrm{CBC})$. Synthesizing boron carbide with high carbon content beyond the solubility limit, in which at. $\% \mathrm{C} \gtrsim 20$, generally results in a mixture of boron carbide with the composition, close to $\mathrm{B}_{4} \mathrm{C}$, and free graphite-like carbon $[36,79,80]$, although a synthesis of boron carbide beyond the carbon-rich limit (at.\% $\mathrm{C} \sim 24$ ) were reported one time in the literature [81]. A recent theoretical study of boron carbide [82, 83] suggested also a possibility of extending the solubility limit of carbon in boron carbide to 28.57 at.\% C. Instead of $\mathrm{B}_{11} \mathrm{C}^{p}(\mathrm{CBC})$, the basic structural units of the proposed carbon-rich boron carbides were given either by $\mathrm{B}_{11} \mathrm{C}^{p}(\mathrm{CC})$ or by $\mathrm{B}_{12} \mathrm{C}_{2}^{p}(\mathrm{CC})$, where $(\mathrm{CC})$ stands for a diatomic chain of carbon atoms. Both $\mathrm{B}_{11} \mathrm{C}^{p}(\mathrm{CC})$ and $\mathrm{B}_{12} \mathrm{C}_{2}^{p}(\mathrm{CC})$ were predicted from first-principles calculations to be metastable with respect to $\mathrm{B}_{11} \mathrm{C}^{p}(\mathrm{CBC})$. These studies thus give rise to suspicion of the maximum carbon content of boron carbide $[20,36,45,57,81,82]$. Moreover, a recent experimental study of boron carbide under high pressure [84] demonstrated a structural phase transition in $\mathrm{B}_{4.3} \mathrm{C}$ at $\sim 40 \mathrm{GPa}$. However, a detailed understanding about the structural change of $\mathrm{B}_{4.3} \mathrm{C}$ under such high pressures has not yet been achieved. It is worth noting that high-pressure experimentation of boron carbide is often done in a diamond anvil cell [84-86], which highlights the importance of investigating the thermodynamics stability of boron carbide in a carbon-rich environment, as it could react with carbon under high pressure and high temperature.

In order to clarify the unresolved questions, regarding the maximum carbon content as well as the stability under high temperature and high pressure of boron carbide, the thermodynamics stability of carbon-rich boron carbide at different compositions, ranging from 20 to 33.33 at.\% C, is inspected as a function of temperature and pressure, using first-principles calculations (Paper III).

\subsection{Boron suboxide}

Similar to boron carbide, boron suboxide $\left(\mathrm{B}_{6} \mathrm{O}\right)$ is also considered as a derivative of $\alpha$-rhombohedral boron [7,8]. Its structural unit can be represented by a $\mathrm{B}_{12}$ icosahedron and a pair of isolated oxygen atoms, denoted by $\mathrm{B}_{12}(\mathrm{O}-\mathrm{O})$, where "-" stands for a vacancy residing between the two oxygen atoms, as can be seen from Fig.1.1(d). Despite having a potential as a superhard material [24, 25], which is highly desirable in many industrial applications, such as cutting tool or protective hard and wear resistant coatings, $\mathrm{B}_{6} \mathrm{O}$ is not yet commercially available. The major problem can be attributed to the difficulty in densifying the material at ambient pressure $[25,87]$. As a consequence, the as-synthesized $\mathrm{B}_{6} \mathrm{O}$ is generally oxygen-deficient, denoted by $\mathrm{B}_{6} \mathrm{O}_{x}$ where $x<0.9$, and has rather poor crystalline quality. Even though high-pressure sintering techniques can enhance the densification of $\mathrm{B}_{6} \mathrm{O}$, thus improving the hardness of the material, its fracture toughness is rather low [88]. A possible solution to overcome the problem of low fracture toughness without sacrificing the high hardness of $\mathrm{B}_{6} \mathrm{O}$, has recently been sug- 
gested by fabricating composites of boron suboxide and boron carbide, using the spark plasma sintering (SPS) method [87]. Although several experimental studies $[87,89,90]$ have so far been made on the composites of boron suboxide and boron carbide, the mixing thermodynamics of these two structurally similar compounds have never been explored.

In this thesis, the thermodynamics of mixing between boron carbide $\mathrm{B}_{13} \mathrm{C}_{2}$ and boron suboxide $\mathrm{B}_{6} \mathrm{O}$, denoted by $\left(\mathrm{B}_{6} \mathrm{O}\right)_{1-x}\left(\mathrm{~B}_{13} \mathrm{C}_{2}\right)_{x}$, as well as their elastic properties are investigated, using first-principles calculations (Paper IV). Theoretical models of the pseudo-binary $\left(\mathrm{B}_{6} \mathrm{O}\right)_{1-x}\left(\mathrm{~B}_{13} \mathrm{C}_{2}\right)_{x}$ alloys with different compositions $x$ are obtained from the SA-SQS method. The knowledge of configurational disorder induced by low-energy substitutional defects, gained from the previous studies of boron carbide (Paper I and Paper II), is also taken into account, when determining the thermodynamic stability of the $\left(\mathrm{B}_{6} \mathrm{O}\right)_{1-x}\left(\mathrm{~B}_{13} \mathrm{C}_{2}\right)_{x}$ alloys and their elastic properties.

\subsection{Boron subarsenide and subphosphide}

Owing to their wide electronic band gaps $\left(E_{g}>3 \mathrm{eV}\right)$, boron subarsenide $\left(\mathrm{B}_{12} \mathrm{As}_{2}\right)$ and boron subphosphide $\left(\mathrm{B}_{12} \mathrm{P}_{2}\right)$ are both promising materials for fabrication of high-temperature electronic devices [27]. Furthermore, due to their self-healing property resistant to structural damage, when subjected to high-energy electron radiation, $\mathrm{B}_{12} \mathrm{As}_{2}$ and $\mathrm{B}_{12} \mathrm{P}_{2}$ are candidates for radiation resistant applications [91, 92], including beta-voltaic devices [93]. Recently, $\mathrm{B}_{12} \mathrm{As}_{2}$ has been suggested as a candidate for thermoelectric applications at high temperature [29, 30]. Since $\mathrm{B}_{12} \mathrm{As}_{2}$ and $\mathrm{B}_{12} \mathrm{P}_{2}$ are isostructural and isovalent, it would perhaps be possible for them to form alloys with limited change in their electronic properties. Taking into account those facts, together with the large mass difference between As and $\mathrm{P}$ atoms giving high alloy phonon scattering, alloying $\mathrm{B}_{12} \mathrm{As}_{2}$ with $\mathrm{B}_{12} \mathrm{P}_{2}$ to form a solid solution is expected to lower the thermal conductivity of the materials, thus improving their thermoelectric properties. However, neither experimental nor theoretical study of the mixing alloys between $\mathrm{B}_{12} \mathrm{As}_{2}$ with $\mathrm{B}_{12} \mathrm{P}_{2}$ have so far been reported in the literature.

Apart from being synthesized, using the chemical vapor deposition (CVD) technique for instance [27, 94-98], $\mathrm{B}_{12} \mathrm{As}_{2}$ and $\mathrm{B}_{12} \mathrm{P}_{2}$ can also be obtained from the irreversible thermal decompositions of zinc-blende boron arsenide (BAs) and boron phosphide $(\mathrm{BP})$ at high temperature $(T \gtrsim 1200 \mathrm{~K})$, respectively [99-101]. BAs, in itself, is also of interest, as it has been predicted to have a remarkably high value of thermal conductivity $(\kappa)$ at room temperature $\left(2240 \mathrm{~W} \cdot \mathrm{m}^{-1} \cdot \mathrm{K}^{-1}\right)$, comparable to that of diamond [102]. Recent studies have, however, demonstrated that the experimental values of $\kappa$ of BAs are approximately 10 times smaller than the theoretical value $\left(\sim 200 \mathrm{~W} \cdot \mathrm{m}^{-1} \cdot \mathrm{K}^{-1}\right)[103,104]$. Such a reduction in $\kappa$ of BAs has been suggested to be originating from the presence of As vacancies, since the assynthesized BAs samples were As-deficient [103-105]. Consequently, synthesizing idealized stoichiometric BAs with high crystalline quality is still a practical challenge, which is questionable whether BAs is a stable compound in the binary B-As 
system. Note that the thermodynamic stability of BAs has been rarely discussed in the literature [106]. In addition, a pseudo-binary phase diagram of zinc-blende $\mathrm{BAs}_{1-x} \mathrm{P}_{x}$ alloys, previously suggested to explain their mixing thermodynamics [107], was sketched without considering $\mathrm{B}_{12} \mathrm{As}_{2}$ and $\mathrm{B}_{12} \mathrm{P}_{2}$ as competing phases of $\mathrm{BAs}_{1-x} \mathrm{P}_{x}$ alloys. It is thus questionable if the proposed phase diagram can provide a proper description of the thermodynamic stability of $\mathrm{BAs}_{1-x} \mathrm{P}_{x}$ alloys at elevated temperature.

In this thesis, a theoretical study of mixing thermodynamics between $\mathrm{B}_{12} \mathrm{As}_{2}$ and $\mathrm{B}_{12} \mathrm{P}_{2}$ is performed, based on first-principles calculations. Similar to the pseudo-binary $\left(\mathrm{B}_{6} \mathrm{O}\right)_{1-x}\left(\mathrm{~B}_{13} \mathrm{C}_{2}\right)_{x}$ alloys, the structural models of the mixing alloys between $\mathrm{B}_{12} \mathrm{As}_{2}$ and $\mathrm{B}_{12} \mathrm{P}_{2}$ are also obtained from the SA-SQS method. The thermodynamic stabilities of the zinc-blende $\mathrm{BAs}, \mathrm{BP}$, and $\mathrm{BAs}_{1-x} \mathrm{P}_{x}$ alloys are then reevaluated, by which the icosahedral phases, their constituent elements as well as $\mathrm{A}_{1-x} \mathrm{P}_{x}$ alloys are taking into consideration. Results obtained from the study of different compounds and alloys in the ternary B-As-P system are presented and discussed in Paper V.

\subsection{Boron subnitride}

Compared to the other icosahedral boron-rich solids, in particular boron carbide, having been frequently addressed in the literature $[1,6,17,20,25,27,28,30,35$, $36,84,91,94-96,108-114]$, relatively few studies have so far been made on boron subnitride [19, 115-120]. For this reason, several issues about boron subnitride, for example its stable compositions, properties, as well as the correct representation of its basic structural unit, are still inconclusive. Up until now, three different compositions of boron subnitride have been proposed in the literature, based on both experimental analyses [117-119] and theoretical calculations [19]. Those are, $\mathrm{B}_{6} \mathrm{~N}$ represented by $\mathrm{B}_{12}(\mathrm{~N}-\mathrm{N})$ [117], $\mathrm{B}_{13} \mathrm{~N}_{2}$ represented by $\mathrm{B}_{12}(\mathrm{NBN})$ [118-120], and $\mathrm{B}_{38} \mathrm{~N}_{6}$ represented by $\left[\mathrm{B}_{12}(\mathrm{~N}-\mathrm{N})\right]_{0.33}\left[\mathrm{~B}_{12}(\mathrm{NBN})\right]_{0.67}[19]$. Note that the basic structural units of $\mathrm{B}_{6} \mathrm{~N}$ and $\mathrm{B}_{13} \mathrm{~N}_{2}$ are analogous to those of $\mathrm{B}_{6} \mathrm{O}$ and $\mathrm{B}_{13} \mathrm{C}_{2}$, respectively, whiles $\mathrm{B}_{38} \mathrm{~N}_{6}$ is represented as a mixture between $\mathrm{B}_{12}(\mathrm{NBN})$ and $\mathrm{B}_{12}(\mathrm{~N}-\mathrm{N})$ with a ratio of $2: 1$. Due to the small variation of lattice parameters of the as-synthesized boron subnitrides found in experiments, it has been suggested that boron subnitride, instead of a solid solution, is an individual line compound [119]. Furthermore, due to the incompleteness of the reactions, the as-synthesized boron subnitrides had often poor crystalline quality, and were always found in a mixture with $\beta$-rhombohedral boron, hexagonal boron nitride and $\alpha$-tetragonal boron subnitride, limiting accessibility to the properties of boron subnitride in experiments [119, 120].

This thesis also aims at clarifying the thermodynamic stability of $\mathrm{B}_{6} \mathrm{~N}, \mathrm{~B}_{13} \mathrm{~N}_{2}$, and $\mathrm{B}_{38} \mathrm{~N}_{6}$ at different pressures and temperatures in order to determine the stable compositions of boron subnitride as well as their stability ranges, using firstprinciples methods. Some intrinsic properties of the three boron subnitrides are also derived. Results obtained from the study of boron subnitride are presented and discussed in Paper VI. 


\section{CHAPTER 2}

\section{Density functional theory}

\subsection{Theoretical background}

The advent of quantum mechanics with the concept of wave-particle duality allowed one to describe the microscopic properties of condensed matters by solving the famous time-dependent Schrödinger equation (in Hartree atomic units);

$$
i \frac{\partial \Psi}{\partial t}=\hat{H} \Psi
$$

where $\Psi$ is the wave function containing all the information of any system under consideration. Naturally, all matter is made up of a large number of microscopic particles, typically nuclei and electrons interacting with each other. Therefore, one can write down the Hamiltonian $\hat{H}$ of such a system, given by;

$\hat{H}=-\frac{1}{2} \sum_{i=1}^{n} \nabla_{i}^{2}-\frac{1}{2} \sum_{I=1}^{N} \frac{1}{M_{I}} \nabla_{I}^{2}-\sum_{i, I} \frac{Z_{I}}{\left|\mathbf{r}_{i}-\mathbf{R}_{I}\right|}+\frac{1}{2} \sum_{i \neq j} \frac{1}{\left|\mathbf{r}_{i}-\mathbf{r}_{j}\right|}+\frac{1}{2} \sum_{I \neq J} \frac{Z_{I} Z_{J}}{\left|\mathbf{R}_{I}-\mathbf{R}_{J}\right|}$.

The first two terms in Eq.(2.2) are the kinetic energy of electrons and of nuclei, respectively, where $M_{I}$ denotes the mass of the nucleus at site $I$. Since both the nuclei and electrons are charged particles, the following three terms represent the potential energy due to the Coulomb interactions between electron-nucleus, electron-electron, and nucleus-nucleus, respectively. The vector $\mathbf{r}_{i}$ refers to the position of electron at site $i$, meanwhile $\mathbf{R}_{I}$, and $Z_{I}$ stand for the position and the charge number of the nucleus at site $I$, respectively.

Solving Eq.(2.1) for a general system of condensed matters is impractical at the moment, since a macroscopic system consists of a number of particles on the order of Avogadro's number. The wave function $\Psi$ describing the system thus depends 
on a huge number of degrees of freedom, and can be expressed by;

$$
\Psi=\Psi\left(\mathbf{r}_{1}, \mathbf{r}_{2}, \ldots, \mathbf{r}_{n}, \sigma_{1}, \sigma_{2}, \ldots, \sigma_{n}, \mathbf{R}_{1}, \mathbf{R}_{2}, \ldots, \mathbf{R}_{N}, t\right),
$$

where $\sigma_{i}$ denotes the spin of the electron at site $i$. To handle such difficulties, several approximations are involved in order to simplify the problem. Mostly, the stationary ground state properties of the system are of main interest. In this case, there is no explicit time dependence in the Hamiltonian. Therefore, only the timeindependent Schrödinger equation alone is adequate to describe the system. The time-independent Schrödinger equation can be written as;

$$
\hat{H} \Psi=E_{t o t} \Psi .
$$

The wave function $\Psi$ is now time-independent;

$$
\Psi=\Psi\left(\mathbf{r}_{1}, \mathbf{r}_{2}, \ldots, \mathbf{r}_{n}, \sigma_{1}, \sigma_{2}, \ldots, \sigma_{n}, \mathbf{R}_{1}, \mathbf{R}_{2}, \ldots, \mathbf{R}_{N}\right)
$$

and is a set of solutions for the eigenvalue equation (Eq.(2.4)), which represents the stationary states of $\hat{H}$ with the corresponding total energies $E_{t o t}$. The next simplification to the problem is the so-called Born-Oppenheimer approximation [121]. The approximation is based on the fact that the nuclei are moving much slower than the electrons because of their several orders of magnitude larger masses. One can assume, at this point, that the nuclei are fixed from the electrons' point of view. As a result, the kinetic term of the nuclei can be separated out, and the nucleus-nucleus interaction term in Eq.(2.2) becomes a constant $\left(E_{I I}\right)$, and can as well be taken care of separately. The problem now reduces to the system of interacting electrons, and the electron-nucleus interaction term is treated as an external field acting on the electrons. The eigenvalue equation of the electronic system is given by;

$$
\hat{H}_{\text {elec }} \Psi_{\text {elec }}=E_{\text {elec }} \Psi_{\text {elec }} .
$$

in which $\Psi_{\text {elec }}$ is the wave function of the system of $n$-interacting electrons in the external field of the fixed nuclei;

$$
\Psi_{\text {elec }}=\Psi_{\text {elec }}\left(\mathbf{r}_{1}, \mathbf{r}_{2}, \ldots, \mathbf{r}_{n}, \sigma_{1}, \sigma_{2}, \ldots, \sigma_{n}\right),
$$

and $\hat{H}_{\text {elec }}$ is the electronic Hamiltonian, given by;

$$
\hat{H}_{\text {elec }}=-\frac{1}{2} \sum_{i=1}^{n} \nabla_{i}^{2}+\frac{1}{2} \sum_{i \neq j} \frac{1}{\left|\mathbf{r}_{i}-\mathbf{r}_{j}\right|}-\sum_{i, I} \frac{Z_{I}}{\left|\mathbf{r}_{i}-\mathbf{R}_{I}\right|} .
$$

The eigenvalues $E_{\text {elec }}$ represents the corresponding energies of the electronic system. The total energy $\left(E_{t o t}\right)$ of the system, given in Eq.(2.4), can thus be calculated from;

$$
E_{\text {tot }}=E_{\text {elec }}+E_{I I}-\frac{1}{2} \sum_{I=1}^{N} \frac{1}{M_{I}} \nabla_{I}^{2} .
$$

The problem can be further simplified if the system under consideration is periodic, such as crystalline solids. As demonstrated by Bloch [122], due to periodicity 
of the crystal, it is sufficient to consider only the primitive unitcell consisting of a few particles, rather than taking into account a large number of particles, representing the macroscopic crystal. The wave functions $\Psi_{\text {elec }}$, which are the solutions to Eq.(2.6), can thus be taking the form;

$$
\psi_{n \mathbf{k}}(\mathbf{r})=e^{i \mathbf{k} \cdot \mathbf{r}} u_{n \mathbf{k}}(\mathbf{r}),
$$

where $n$ is the band index and takes numbers $n=1,2,3, \ldots$ and $\mathbf{k}$ is a reciprocal vector, principally in the first Brillouin zone. $u_{n \mathbf{k}}(\mathbf{r})$ is a function with periodicity of the crystal lattice;

$$
u_{n \mathbf{k}}(\mathbf{r}+\mathbf{T})=u_{n \mathbf{k}}(\mathbf{r}),
$$

where $\mathbf{T}$ is a translation vector of the lattice. $e^{i \mathbf{k} \cdot \mathbf{r}}$ describes a plane wave. Even though the problem, at this point, is much simplified through the above approximations, they are still impractical to directly solve Eq.(2.6). This is because solving the Schrödinger equation for a system containing more than a few particles is in practice a formidable task.

\subsection{The Hohenberg-Kohn theorems}

Instead of solving for the many-electron wave function $\Psi_{\text {elec }}$ in Eq.(2.6), one can use the electron density $n(\mathbf{r})$ as a basic variable. For a system of $n$-interacting electrons, such a change of the variable reduces the number of spatial coordinates from $3 n$ to 3 , and thus substantially simplifies the problem. The attempt to use $n(\mathbf{r})$ as a basic variable was first proposed in 1927 and is known as the Thomas-Fermi theory $[123,124]$. Unfortunately, results calculated from the theory were inaccurate in most applications, since the many-particle interactions between the electrons, i.e. exchange and correlation, were completely neglected, and the kinetic energy were approximated far too much. In 1964, Hohenberg and Kohn formulated two theorems [125], based on $n(\mathbf{r})$, that has been seen as the starting point of modern density functional theory (DFT). The two theorems are stated [126] as follows;

\section{Theorem I}

For any system of interacting particles in an external potential $V_{\text {ext }}(\mathbf{r})$, the potential $V_{\text {ext }}(\mathbf{r})$ is determined uniquely, except for a constant, by the ground state particle density $n_{0}(\mathbf{r})$.

\section{Theorem II}

A universal function for the energy $E[n]$ in term of the density $n(\mathbf{r})$ can be defined, valid for any external potential $V_{\text {ext }}(\mathbf{r})$. For any particular $V_{\text {ext }}(\mathbf{r})$, the exact ground state energy of the system is the global minimum value of this functional, and the density $n(\mathbf{r})$ the minimizes the functional is the exact ground state density $n_{0}(\mathbf{r})$.

From the two theorems, it is clear that if the exact form of the total energy functional $E[n]$ was known, it could be used through the minimization procedure to exactly find the ground state density $n_{0}(\mathbf{r})$ from which, in principles, all ground 
state properties could be obtained. The total energy functional, in principle, can be expressed as;

$$
E[n]=T[n]+E_{i n t}[n]+\int d^{3} r V_{e x t}(\mathbf{r}) n(\mathbf{r})+E_{I I},
$$

where the first term on the right-hand side is the kinetic energy of the interacting electrons, meanwhile the following three terms represent the potential energies due to the Coulomb interaction between the electrons, the Coulomb interaction of the electron density $n(\mathbf{r})$ with the external field $V_{\text {ext }}(\mathbf{r})$, and the interaction between the nuclei, respectively. However, the remaining problem to solve for the system of $n$-interacting electrons is that the exact forms of $T[n]$ and $E_{\text {int }}$ are not known.

\subsection{Kohn-Sham equations}

In 1965, Kohn and Sham proposed a practical approach [127] to overcome the difficulties in solving for the ground state properties of the interacting many-particle problem. The idea is to replace the real system of interacting particles (Eq.(2.6)) by an artificial system of non-interacting particles, having the same density $n(\mathbf{r})$ as does the real one. Rather than the external potential $V_{\text {ext }}(\mathbf{r})$, each non-interacting particle is subjected to the effective potential $V_{\text {eff }}(\mathbf{r})$, given by;

$$
V_{e f f}(\mathbf{r})=V_{e x t}(\mathbf{r})+\int \frac{n\left(\mathbf{r}^{\prime}\right)}{\left|\mathbf{r}-\mathbf{r}^{\prime}\right|} d \mathbf{r}^{\prime}+V_{x c}(\mathbf{r})
$$

The second term on the right-hand side of Eq.(2.13) is the so-called Hartree potential, describing the Coulomb interaction between the electrons, as in the case of a self-interacting classical charged density, whiles the term $V_{x c}(\mathbf{r})$ is defined as the exchange-correlation potential, accounting for all the quantum mechanical and explicit many-body effects, and can be calculated from the exchange-correlation energy functional $E_{x c}[n(\mathbf{r})]$ via;

$$
V_{x c}(\mathbf{r})=\frac{\partial E_{x c}[n(\mathbf{r})]}{\partial n(\mathbf{r})} .
$$

The non-interacting particles can thus be described by the so-called Kohn-Sham wave functions $\psi_{i}$, which are the eigenstates to the single-particle Schrödinger-like equation;

$$
\left(-\frac{1}{2} \nabla^{2}+V_{e f f}(\mathbf{r})\right) \psi_{i}(\mathbf{r})=\epsilon_{i} \psi_{i}(\mathbf{r})
$$

Eq.(2.15) is known as the Kohn-Sham equation. $\epsilon_{i}$ is the eigenvalue of the noninteracting single particle, corresponding to the Kohn-Sham eigenstate $\psi_{i}$. Within the Kohn-Sham scheme, the particle density $n(\mathbf{r})$ of a system with $N$ non-interacting particles can be easily obtained as;

$$
n(\mathbf{r})=\sum_{i=1}^{N}\left|\psi_{i}(\mathbf{r})\right|^{2} .
$$


The Kohn-Sham total energy functional is given by;

$$
E_{K S}[n]=T_{s}[n]+\int d^{3} r V_{e x t}(\mathbf{r}) n(\mathbf{r})+E_{\text {Hartree }}[n]+E_{x c}[n]+E_{I I},
$$

where $T_{s}[n]$ is the kinetic energy functional of the non-interacting particles;

$$
T_{s}[n]=-\frac{1}{2} \sum_{i=1}^{N}\left\langle\psi_{i}\left|\nabla^{2}\right| \psi_{i}\right\rangle,
$$

and $E_{\text {Hartree }}[n]$ is the classical Coulomb energy functional due to the particle density $n(\mathbf{r})$, interacting with itself. The expression of the $E_{\text {Hartree }}[n]$ functional is given by;

$$
E_{\text {Hartree }}[n]=\frac{1}{2} \int \frac{n(\mathbf{r}) n\left(\mathbf{r}^{\prime}\right)}{\left|\mathbf{r}-\mathbf{r}^{\prime}\right|} d \mathbf{r} d \mathbf{r}^{\prime}
$$

\subsection{Exchange-correlation functional}

The major problem to solve the Kohn-Sham equation (Eq.(2.15)) is that the form of the exchange-correlation functional $E_{x c}[n]$ is not known exactly. However, it is still possible to formulate the functional with some approximations. Several approaches to estimate $E_{x c}[n]$ have been proposed and constantly developed in order to improve the accuracy. Some are given as follows;

\section{Local density approximation (LDA)}

The local density approximation (LDA) is the simplest way to derive $E_{x c}[n]$. LDA was first suggested at the same time as the Kohn-Sham equation was formulated in 1965 [127]. In practice, LDA approximates $E_{x c}[n]$ by assuming that the exchange-correlation energy density $\epsilon_{x c}^{L D A}(n(\mathbf{r}))$ at each point $\mathbf{r}$ in space is of the same form as the homogeneous electron gas $\epsilon_{x c}^{\text {hom }}(n)$, which has been well-studied using quantum Monte Carlo simulations [128]. The expression of $E_{x c}^{L D A}[n]$ is thus given by;

$$
E_{x c}^{L D A}[n]=\int n(\mathbf{r}) \epsilon_{x c}^{h o m}(n(\mathbf{r})) d \mathbf{r} .
$$

At first, LDA was supposed to work well only for system with slowly varying density, as it is exact only for the homogeneous electron gas. However, it was found to be successful also for systems with high density gradients. This is due in part to cancellation of errors in exchange and correlation to a large degree. LDA also fulfills the sum rule for the exchange-correlation hole, integrating over space exactly to -1 per electron. Further explanation to the relative success of LDA have been described elsewhere [126, 129].

\section{Generalized gradient approximation (GGA)}

Even though LDA has turned out to be successful in predicting the properties of solid materials, it in many cases underestimates the lattice spacing. The idea, to improve the accuracy by taking into account not only the density $n(\mathbf{r})$ 
but also the gradient of the density $\nabla n(\mathbf{r})$ at the same point $\mathbf{r}$ to approximate $E_{x c}[n]$, were thus proposed [130-132] as an extension of LDA, which is a so-called generalized gradient approximation (GGA). Consequently,

$$
E_{x c}^{G G A}[n]=\int n(\mathbf{r}) \epsilon_{x c}^{G G A}(n(\mathbf{r}), \nabla n(\mathbf{r})) d \mathbf{r} .
$$

Most of the calculations in this thesis are performed using the GGA, proposed by Perdew, Burke, Ernzerhof (PBE96) [132], for the exchange-correlation functional.

\section{Hybrid functionals}

In 1982, Perdew et al. [133] demonstrated, for a system of $N$-interacting electrons, the derivative discontinuities found in the curve of energy $E$ as a function of the number of electrons $N$. The discontinuities, in principle, result in a jump by a positive constant of the exchange-correlation potential $V_{x c}(\mathbf{r})$, as the number of electrons changes by an integer. However, the approximation of $E$ using semilocal functionals, e.g. LDA and GGA, cannot displays such features in the energy curve. As a result, electronic band gaps of solid materials, calculated within the Kohn-Sham scheme, are systematically underestimated with respect to experiment [134]. This problem can be partially corrected by mixing a portion of Hartree-Fock (HF) exchange with those of the semi-local functionals in calculating $E_{x c}[n]$. This concept thus becomes the basics of hybrid functionals. Up to now, several versions of hybrid functionals have been proposed, such as B3LYP [135, 136], PBE0 [137], and HSE [138-140]. Note that by including the HF exchange, one ends up with an orbital $\left(\psi_{i}\right)$-dependent potential, which in turn drastically increases the computational demand, thus practically limiting the size of electronic systems possible to treat in the calculations. In this thesis, the hybrid functional HSE06 [138-140] is used in order to investigate the electronic properties of the presumed $\mathrm{B}_{11} \mathrm{C}^{p}(\mathrm{CBC})$ and $\mathrm{B}_{12}(\mathrm{CBC})$ for $\mathrm{B}_{4} \mathrm{C}$ (Paper $\mathrm{I}$ ) and $\mathrm{B}_{13} \mathrm{C}_{2}$ (Paper II), respectively.

\section{Modified Becke-Johnson (MBJ) exchange potential}

Compared to the HF exchange, the modified Becke-Johnson (MBJ) exchange potential $[141,142]$ requires much less computational resources to perform the calculations, while still claiming to reproduce accurate band gaps, at the same level as the hybrid functionals. Although the MBJ exchange is a semi-local potential, similar to LDA and GGA, it is designed to mimic the behavior of those orbital-dependent potentials, i.e. the derivative discontinuities of the HF exchange potential. However, one needs to keep in mind that the MBJ functional exists only in the potential form $V_{x}(\mathbf{r})$, and there is no corresponding exchange energy functional $E_{x}[n]$. Therefore, the self-consistent calculations of the total energy $E_{K S}[n]$ will never be achieved with the MBJ potential. In this thesis, the MBJ exchange potential was used in combination with the GGA-PBE96 correlation [132] to calculate the electronic band gaps of $\mathrm{B}_{4} \mathrm{C}$ (Paper I) and $\mathrm{B}_{13} \mathrm{C}_{2}$ (Paper II). 


\subsection{Plane-wave basis set}

To numerically solve the Kohn-Sham equations in Eq.(2.15), the electron wave functions $\psi_{i}(\mathbf{r})$ need to be expanded in a basis set. Various choices of basis sets are available, such as atomic orbitals, Gaussians, and plane waves. According to the Bloch's theorem [122], the plane wave is considered as the first choice among the others for crystalline solids, as it has been demonstrated that the wave function of any periodic system can, in principle, be expanded in term of a plane wave basis set, see Eq.(2.10). The plane wave basis set, in practice, needs to be truncated at a finite cutoff energy. Based on the characteristics of the electron wave function, it may be splitting in space into (1) an interstitial and (2) a core regions. In the interstitial region between the atoms, where the electrons are delocalized (valence electrons), the wave function is rather smooth and only a small number of plane-waves is already sufficient to well reproduce the wave function. On the other hand, a huge number of plane waves with very high energy cutoff is required to accurately represent the rapidly oscillating wave function inside the core region due to the strong Coulomb interactions between the highly localized core electrons and the nucleus, thus resulting in an extremely high computational cost.

To implement the plane wave basis sets in practice, one employs a pseudopotential (PP) approach, first suggested by Hellmann [143, 144]. The PP approach substitutes the real Coulomb potential by a smooth effective potential, specially designed in order to smoothen the rapidly oscillating part of the wave function within the core region (a pseudo wave function), thus substantially reducing both the basis sets and the computational demand. Inspired by the fact that only the valence electrons residing in the interstitial region are taking part in the chemical bond formation, eliminating the oscillating part within the core region should not be affecting the bonding properties. Within the $\mathrm{PP}$ approach, the core region is determined by a certain cutoff radius from the nucleus. A few criteria to construct the pseudopotential are given as follows; (1) the pseudopotential needs to reproduce the scattering properties of the core region, and (2) outside the cutoff radius, the behavior of the pseudopotential and the pseudo wave function must be the same to the real ones. Among numerously developed versions of the pseudopotentials suggested in the literature, Norm-conserving [145] and Ultrasoft [146] pseudopotentials are most widely used PP in plane wave basis set-based DFT calculations.

A generalization of the pseudopotential approach, also known as projector augmented wave method (PAW), was later proposed by Blöchl [147]. Instead of the pseudo wave function as obtained in the PP approach, the PAW method restores the full real electron wave function $\psi_{i}(\mathbf{r})$ at the end of the calculation. As the PAW method uses the smooth wave function within the core region, like the PP approach, the computational costs of these two approach are comparably cheap. At the same time, due to the restoration of the real wave function, the reliability and the accuracy of the PAW method are similar to those of all electron, and full potential Kohn-Sham approaches. All the calculations in this thesis are performed using the PAW method. 


\subsection{Solving the Kohn-Sham equations}

To search for the ground state density $n_{0}(\mathbf{r})$, minimizing the Kohn-Sham total energy functional $E_{K S}[n]$ in Eq.(2.17), the Kohn-Sham equations, given by Eq.(2.15) must be solved self-consistently. Since the effective potential $V_{\text {eff }}$ and the KohnSham orbitals $\psi_{i}$ in Eq.(2.15) are determined by the density $n(\mathbf{r})$, which is not known a priori, several iterative cycles of solving the Kohn-Sham equations must be performed as illustrated in Fig.2.1. The first step to solve the equation is to guess the initial density $n_{\text {guess }}(\mathbf{r})$ in order to construct $V_{\text {eff }}$, according to Eq.(2.13). After solving the Kohn-Sham equation, one obtains a set of $\psi_{i}$, which is in turn used to calculate a new electron density $\tilde{n}_{k+1}(\mathbf{r})$. If the convergence criterion is not fulfilled, $\tilde{n}_{k+1}(\mathbf{r})$ will be mixed with the input electron density $n_{k}(\mathbf{r})$ using different numerical mixing schemes, e.g. the Anderson and the Broyden methods [126], to get $n_{k+1}(\mathbf{r})$ as a new input to construct a new $V_{e f f}$, and then another iterative cycle of solving Kohn-Sham equation is performed. Such a process continues until the electron density converges and self-consistency is thus reached.

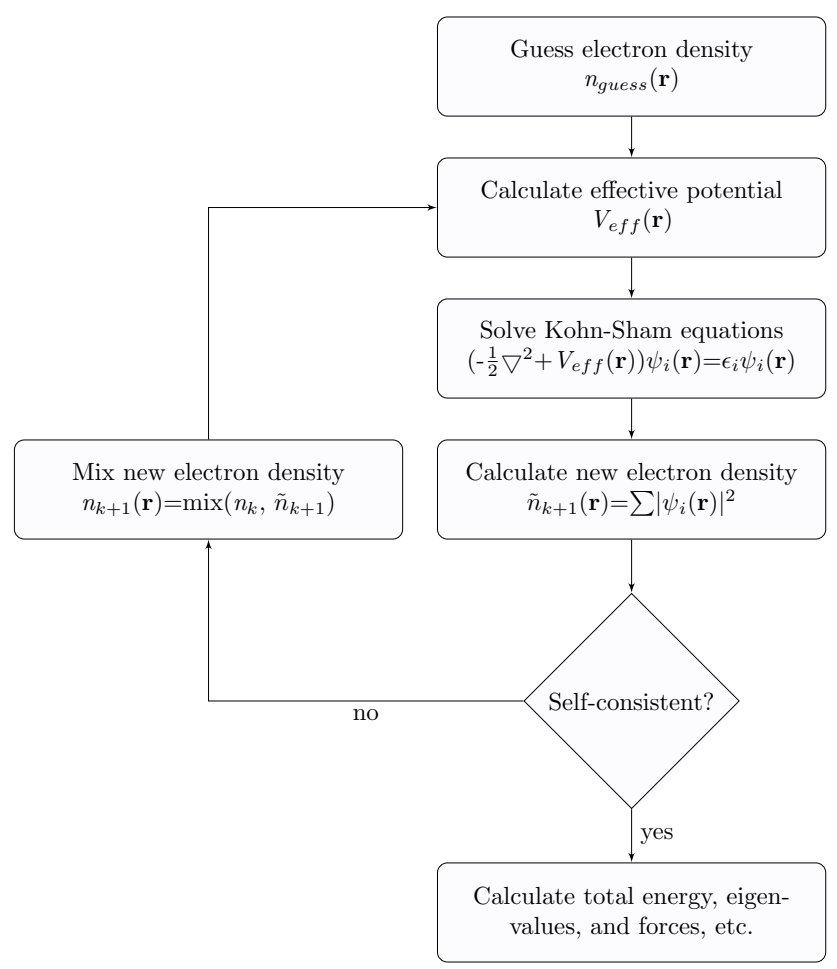

Figure 2.1. Flowchart showing the self-consistent loop for solving Kohn-Sham equations. After Martin [126]. 


\section{CHAPTER 3}

\section{Lattice dynamics}

Due to the use of the Born-Oppenheimer approximation to simplify the problem in Chapter 2, the atomic nuclei in a crystalline solid are treated as static particles, exactly positioned at the lattice sites, generally referred to as equilibrium positions. Indeed, this is an approximation, since in reality the nuclei are collectively vibrating about their equilibrium positions, resulting in the so-called lattice $d y$ namics. Understanding the lattice dynamics is crucial, as it can have a significant impact on the stability and properties of the materials at finite temperature.

\subsection{Introduction}

The fundamental theory of lattice dynamics was originally formulated from the classical point of view by Born and von Kármán [148]. In this case, atoms residing in the lattice sites are assumed to be interconnected by massless springs, and thus the forces acting on the atoms are proportional to the relative displacements of the atoms, analogous to simple harmonic oscillators. Considering a crystal, whose unitcell is defined by the three basic lattice vectors $\boldsymbol{a}_{1}, \boldsymbol{a}_{2}$, and $\boldsymbol{a}_{3}$, any lattice point in the crystal can be described by a lattice translation operator $T$, which is a linear combination of the three basic vectors,

$$
T=\mu_{1} \boldsymbol{a}_{1}+\mu_{2} \boldsymbol{a}_{2}+\mu_{3} \boldsymbol{a}_{3},
$$

where $\mu_{1}, \mu_{2}$, and $\mu_{3}$ are integers. If the equilibrium position of the atom $\nu$ in the unitcell, with respect to the origin of the unitcell, is defined as a vector $\boldsymbol{r}^{\prime}(\nu)$, the position of the atom $\nu$ residing in the unitcell $\mu$ of the crystal can be given by;

$$
\boldsymbol{r}(\mu \nu)=\boldsymbol{T}(\mu)+\boldsymbol{r}^{\prime}(\nu) .
$$


A displacement $\boldsymbol{u}(\mu \nu)$ of the atom $\mu \nu$ from its equilibrium position, in general, results in an increase in the potential energy $U$ of the crystal lattice, which can be expressed as a function of the displacement $\{\boldsymbol{u}\}$, using the Taylor series expansion [149];

$$
\begin{aligned}
& U(\{\boldsymbol{u}\})=U_{0}+\sum_{\mu \nu \alpha} \Phi_{\alpha}(\mu \nu) u_{\alpha}(\mu \nu)+\frac{1}{2 !} \sum_{\mu \nu \alpha} \sum_{\mu^{\prime} \nu^{\prime} \beta} \Phi_{\alpha \beta}\left(\mu \nu, \mu^{\prime} \nu^{\prime}\right) u_{\alpha}(\mu \nu) u_{\beta}\left(\mu^{\prime} \nu^{\prime}\right) \\
& +\frac{1}{3 !} \sum_{\mu \nu \alpha} \sum_{\mu^{\prime} \nu^{\prime} \beta} \sum_{\mu^{\prime \prime} \nu^{\prime \prime} \gamma} \Phi_{\alpha \beta \gamma}\left(\mu \nu, \mu^{\prime} \nu^{\prime}, \mu^{\prime \prime} \nu^{\prime \prime}\right) u_{\alpha}(\mu \nu) u_{\beta}\left(\mu^{\prime} \nu^{\prime}\right) u_{\gamma}\left(\mu^{\prime \prime} \nu^{\prime \prime}\right)+\cdots
\end{aligned}
$$

$U_{0}$ is the potential energy of the crystal, in which all atoms are located at their equilibrium positions. The Taylor expansion coefficients;

$$
\begin{gathered}
\Phi_{\alpha}(\mu \nu)=\left.\frac{\partial U}{\partial u_{\alpha}(\mu \nu)}\right|_{\boldsymbol{u}=0}=0 \\
\Phi_{\alpha \beta}\left(\mu \nu, \mu^{\prime} \nu^{\prime}\right)=\left.\frac{\partial^{2} U}{\partial u_{\alpha}(\mu \nu) \partial u_{\beta}\left(\mu^{\prime} \nu^{\prime}\right)}\right|_{\boldsymbol{u}=0} \\
\Phi_{\alpha \beta \gamma}\left(\mu \nu, \mu^{\prime} \nu^{\prime}, \mu^{\prime \prime} \nu^{\prime \prime}\right)=\left.\frac{\partial^{3} U}{\partial u_{\alpha}(\mu \nu) \partial u_{\beta}\left(\mu^{\prime} \nu^{\prime}\right) \partial u_{\gamma}\left(\mu^{\prime \prime} \nu^{\prime \prime}\right)}\right|_{\boldsymbol{u}=0}
\end{gathered}
$$

are referred to as the force constant matrices. The indices $\alpha, \beta$, and $\gamma$ denote the Cartesian coordinates. $u_{\alpha}, u_{\beta}$, and $u_{\gamma}$ refer to the components of the displacement $\boldsymbol{u}(\mu \nu)$ of the atom $\mu \nu$ in the directions of $\alpha, \beta$, and $\gamma$, respectively. Since the force constant matrices $\Phi$, given in Eq.(3.4), are evaluated at the equilibrium positions $\boldsymbol{u}=0$, the first order terms $\Phi_{\alpha}(\mu \nu)$, which explain the net force acting on the atom $\mu \nu$ must be zero.

\subsection{Harmonic approximation}

By Assuming that the amplitudes of the atomic vibrations are sufficiently small around their equilibrium positions, the expression of $U(\{\boldsymbol{u}\})$, given in Eq.(3.3), can be truncated at the second derivatives;

$$
U(\{\boldsymbol{u}\})=U_{0}+\frac{1}{2} \sum_{\mu \nu \alpha} \sum_{\mu^{\prime} \nu \beta} \Phi_{\alpha \beta}\left(\mu \nu, \mu^{\prime} \nu^{\prime}\right) u_{\alpha}(\mu \nu) u_{\beta}\left(\mu^{\prime} \nu^{\prime}\right) .
$$

The truncated potential energy $U(\{\boldsymbol{u}\})$, given in Eq.(3.5), is of the same form as that of the harmonic oscillator, which is the so-called harmonic approximation. The second order force constants $\Phi_{\alpha \beta}\left(\mu \nu, \mu^{\prime} \nu^{\prime}\right)$ can be described as the force acting on the atom $\mu \nu$ in the direction $\alpha$ due to the displacement of atom $\mu^{\prime} \nu^{\prime}$ in the direction $\beta$. Mathematically, they are represented as matrices, consisting of $3 \times 3$ elements. The third and higher order terms, being neglected, can on the one hand be physically assigned as the anharmonic contributions from the lattice dynamics. 
From Eq.(3.5), one can accordingly write down the Hamiltonian $H$, describing behavior of the lattice vibrations, as;

$$
H=U_{0}+\frac{1}{2} \sum_{k l \alpha \beta} \Phi_{\alpha \beta}^{k l} u_{k \alpha} u_{l \beta}+\sum_{k \alpha} \frac{p_{k \alpha}^{2}}{2 m_{k}} .
$$

Note that, starting from Eq.(3.6), the notations of the atomic indices $\mu \nu$ and $\mu^{\prime} \nu^{\prime}$ are shortened to $k$ and $l$, respectively, which will be used for the rest of the texts and equations in Chapter 3. The last term on the right-hand side of Eq.(3.6) is the kinetic energy of atoms due to the vibrations, where $p_{k \alpha}$ and $m_{k}$ denote the Cartesian component $\alpha$ of the momentum and mass of the atom $k$, respectively. By using the relations;

$$
p_{k \alpha}=-\frac{\partial H}{\partial u_{k \alpha}}
$$

and

$$
\dot{u}_{k \alpha}=\frac{\partial H}{\partial p_{k \alpha}},
$$

the equation of motion for the atom $k$ can then be written as;

$$
m_{k} \ddot{u}_{k \alpha}=-\sum_{l \beta} \Phi_{\alpha \beta}^{k l} u_{l \beta} .
$$

For a crystal containing $N$ atoms in a unitcell, there are in total $3 N$ equations of motion, needed to be solved. Due to the periodicity of the crystal lattice, the atomic displacements $u_{k \alpha}$, which is the solutions of the equations of motion, take the form of plane waves, summed over the wavevectors $\boldsymbol{q}$ in the first Brillouin zone;

$$
u_{k \alpha}=\sum_{\boldsymbol{q}} A_{\boldsymbol{q}} \epsilon_{\boldsymbol{q}}^{k \alpha} e^{i(\boldsymbol{q} \cdot \boldsymbol{r}-\omega t)} .
$$

$A, \epsilon$, and $\omega$ are defined as the amplitude of the plane wave, polarization vector, and frequency, respectively. By substituting Eq.(3.10) into Eq.(3.9) and exploiting the orthogonality of the plane waves, one obtains the eigenvalue equation;

$$
D(\boldsymbol{q}) \epsilon=\omega^{2} \epsilon .
$$

$D(\boldsymbol{q})$ is the dynamical matrix, represented by a $3 N \times 3 N$ matrix, and consists of $3 \times 3$ submatrices $D_{k l}^{\alpha \beta}(\boldsymbol{q})$ as its elements, which can be obtained from the Fourier transform of the force constant matrix $\Phi_{\alpha \beta}^{k l}$;

$$
D_{k l}^{\alpha \beta}(\boldsymbol{q})=\sum_{k} \frac{\Phi_{\alpha \beta}^{k l}}{\sqrt{m_{k} m_{l}}} e^{i \boldsymbol{q} \cdot \boldsymbol{r}} .
$$

By solving Eq.(3.11), one obtains the vibrational frequency $\omega_{s, \boldsymbol{q}}$. For each wave vector $\boldsymbol{q}$, there are $3 N$ values of $\omega$ (branches or normal modes), indexed by the subscript $s$. Plotting $\omega_{s, \boldsymbol{q}}$ against $\boldsymbol{q}$ forms the so-called dispersion relation.

Quantum mechanically, the normal modes of lattice vibrations can be considered as quasi-particles, referred to as phonons. To determine the energy, carried by 
the phonons, one introduces the formalism of second quantization to describe the position and momentum in terms of the creation $\hat{a}$ and annihilation $\hat{a}^{\dagger}$ operators [150];

$$
\begin{aligned}
& \hat{u}_{k \alpha}=\sum_{s, \boldsymbol{q}} \sqrt{\frac{\hbar}{2 N m_{k} \omega_{s, \boldsymbol{q}}}} \epsilon_{s, \boldsymbol{q}}^{k \alpha} e^{i \boldsymbol{q} \cdot \boldsymbol{r}}\left(\hat{a}_{s, \boldsymbol{q}}+\hat{a}_{s,-\boldsymbol{q}}^{\dagger}\right) \\
& \hat{p}_{k \alpha}=\sum_{s, \boldsymbol{q}} \frac{1}{i} \sqrt{\frac{\hbar m_{k} \omega_{s, \boldsymbol{q}}}{2 N}} \epsilon_{s, \boldsymbol{q}}^{k \alpha} e^{i \boldsymbol{q} \cdot \boldsymbol{r}}\left(\hat{a}_{s, \boldsymbol{q}}-\hat{a}_{s,-\boldsymbol{q}}^{\dagger}\right) .
\end{aligned}
$$

By substituting $\hat{u}_{k \alpha}$ and $\hat{p}_{k \alpha}$ operators, given in Eq.(3.13), into Eq.(3.6), the vibrational Hamiltonian $H$ becomes an operator, which can be written as;

$$
\hat{H}=\sum_{s, \boldsymbol{q}} \hbar \omega_{s, \boldsymbol{q}}\left(\hat{a}_{s, \boldsymbol{q}}^{\dagger} \hat{a}_{s, \boldsymbol{q}}+\frac{1}{2}\right) .
$$

Since the number operator $\hat{N}$ is defined as $\hat{a}^{\dagger} \hat{a}$, the total energy contributed by the lattice vibrations $E_{v i b}$ can be obtained from;

$$
E_{v i b}=\sum_{s, \boldsymbol{q}} \hbar \omega_{s, \boldsymbol{q}}\left(n_{s, \boldsymbol{q}}+\frac{1}{2}\right),
$$

where $n_{s, \boldsymbol{q}}$ is the number of phonons at a particular mode $s, \boldsymbol{q}$. As for the phonon obeying the Bose-Einstein partition statistics, $n_{s, \boldsymbol{q}}$ thus takes the form;

$$
n_{s, \boldsymbol{q}}=\frac{1}{\exp \left(\hbar \omega_{s, \boldsymbol{q}} / k_{B} T\right)-1} .
$$

$k_{B}$ and $T$ are Boltzmann constant and absolute temperature, respectively. Since the vibrational Hamiltonian $\hat{H}$, given in Eq.(3.14), is a sum of the uncoupled harmonic oscillators, the partition function $\mathcal{Z}$ can be written as;

$$
\mathcal{Z}=\prod_{s, \boldsymbol{q}} \frac{\exp \left(-\hbar \omega_{s, \boldsymbol{q}} / 2 k_{B} T\right)}{1-\exp \left(-\hbar \omega_{s, \boldsymbol{q}} / k_{B} T\right)}
$$

The thermodynamics properties, associated with the phonons, e.g. the vibrational Helmholtz free energy $F_{v i b}$, can thus be derived from the partition function $\mathcal{Z}$;

$$
\begin{aligned}
F_{v i b}(T)=-k_{B} T \ln \mathcal{Z}= & \sum_{s, \boldsymbol{q}} \frac{\hbar \omega_{s, \boldsymbol{q}}}{2}+k_{B} T \sum_{s, \boldsymbol{q}} \ln \left(1-\exp \left(-\frac{\hbar \omega_{s, \boldsymbol{q}}}{k_{B} T}\right)\right) \\
& =\int_{0}^{\infty}\left[g(\omega) \frac{\hbar \omega}{2}+g(\omega) k_{B} \ln \left(1-\exp \left(-\frac{\hbar \omega}{k_{B} T}\right)\right)\right] d \omega,
\end{aligned}
$$

where $g(\omega)$ is defined as the phonon density of states, given by;

$$
g(\omega)=\sum_{s} \int_{\mathrm{BZ}} \frac{\delta\left(\omega-\omega_{s}(\boldsymbol{q})\right)}{2 \pi^{3}} d \boldsymbol{q} .
$$




\subsection{Quasi-harmonic approximation}

Even though the harmonic approximation succeeds in accounting for many thermal properties, associated with the lattice vibrations, it has some limitations. In fact, one manifestation of anharmonicity is that the vibrational frequency $\omega$ and thus the related thermal properties change with the unitcell volume $V$ and temperature $T$ of the crystal. Owing to the absence of the thermal expansion within the harmonic approximation, such volume and temperature dependences of $\omega$ cannot be captured. In addition, the third and higher order terms in Eq.(3.3), representing the anharmonic contributions from the lattice vibrations are completely neglected. Anhamonicity of the lattice vibrations can be of importance at high temperature, as the atoms strongly vibrate, resulting in significantly large displacements from their equilibrium positions. The first order correction to remedy the absence of the thermal expansion can be done by using the harmonic approximation for several different volumes $V$ of the crystal, called quasi-harmonic approximation [151, 152]. In this case, the potential energy $U(\{\boldsymbol{u}\})$ is still in the form of the harmonic approximation (Eq.(3.5)), but it depends also on the volume $V$, which can now be considered as a function of temperature, that is $V(T)$. As a consequence, the vibrational frequency $\omega(V, T)$ can be approximated as $\omega(V(T))$, and the vibrational Helmholtz free energy $F_{v i b}$, given in Eq.(3.18), can now be expressed as a function of volume $V$ and temperature $T$;

$$
F_{v i b}(T, V)=\sum_{s, \boldsymbol{q}} \frac{\hbar \omega_{s, \boldsymbol{q}}(V(T))}{2}+k_{B} T \sum_{s, \boldsymbol{q}} \ln \left(1-\exp \left(-\frac{\hbar \omega_{s, \boldsymbol{q}}(V(T))}{k_{B} T}\right)\right) .
$$

Since the fundamental thermodynamic relation can be expressed in terms of the Helmholtz free energy $F$ by;

$$
d F=-S d T-p d V
$$

the vibrational entropy $S_{v i b}$, describing the number of ways in which the vibrations can be set up in the crystal, and the pressure $p$, exerted on the crystal due to the lattice vibrations, can be obtained, respectively, from;

$$
S_{v i b}(T, V)=-\left.\frac{\partial F_{v i b}(T, V)}{\partial T}\right|_{V}
$$

and

$$
p=-\left.\frac{\partial F_{v i b}(T, V)}{\partial V}\right|_{T} .
$$

In this thesis, the vibrational Helmholtz free energy $F_{v i b}$ is calculated at the quasiharmonic level, and is used for determining the thermodynamic stability of boron carbide (Paper III), boron subnitride (Paper VI), boron pnictides and boron subpnictides (Paper V).

It is worth noting that, within the harmonic and quasi-harmonic approximations, the potential energy $U$, and thus the force constant matrices $\Phi$ are evaluated 
at $T=0 \mathrm{~K}$. However, they can be significantly different at high temperature. Also, the neglect of the anharmonic terms of $U(\{\boldsymbol{u}\})$, given in Eq.(3.3), can result in severe problems for interpretation of the lattice dynamics of a highly anharmonic crystal. For example, if the second derivatives in Eq.(3.4b) are negative, the dynamical matrix $D(\boldsymbol{q})$ will no longer be Hermitian, leading to imaginary vibrational frequencies $\omega$. The imaginary frequencies indicate the dynamical instability of the crystal under consideration at $T=0 \mathrm{~K}$, while in reality, the crystal may be stabilized by the anharmonic contributions at elevated temperature. To take into account such anharmonic effects, one needs to go beyond the harmonic and quasiharmonic approximations by implementing more sophisticated methods, typically based on $a b$ initio molecular dynamics (AIMD). 


\section{CHAPTER 4}

\section{Configurational disorder in crystalline solids}

Instead of dealing with a large number of particles in the macroscopic crystal, the Bloch's theorem [122] allows one to consider only a small primitive unitcell consisting of a few particles due to the periodicity of the crystal. However, taking into account effects of configurational disorder in modeling random alloys, for example, destroys the periodicity of the crystal. As a result, the Bloch's theorem is no longer valid. On average, the symmetry of the underlying lattice is still present and is typically observed experimentally. However, from an atomistic perspective, the disorder needs to be explicitly addressed, that is the so-called alloy problem. The dominant theoretical frameworks to deal with such a problem in first principles calculations are the cluster expansion procedure, originating in the work of Connolly and Williams [153], the special quasirandom structure (SQS) method, suggested by Zunger et al. [41], and the coherent potential approximation (CPA), originally proposed by Soven [154]. The latter is a single-site, effective medium approximation, and is not believed to be of quantitative accuracy for semiconducting materials, or in system, where local lattice relaxation can be strong. Therefore, the CPA is not used in this thesis work. The cluster expansion and SQS formalisms, on the other hand, are introduced below.

\subsection{Modeling of configurational disorder}

In order to account for a contribution of configurational disorder in any crystalline solid, it is necessary to understand the mathematical foundation of cluster expansion, as developed by Sanchez, Ducastelle, and Gratias [38-40] for describing the configurational part of the total energy for a given multicomponent alloy. In this section, an introduction to the concept of cluster expansion is given, based on the review article, written by Ruban and Abrikosov [155]. 
For simplicity, one consider a binary alloy, $A_{1-x} B_{x}$. The atomic configuration of the alloy, $A_{1-x} B_{x}$, is described by spin variables $\sigma_{i}$, where $\sigma_{i}$ takes the values +1 , or -1 if site $i$ is occupied by $A$ - or $B$-type atom, respectively. For an alloy system, consisting of $N$ atomic sites, the atomic configuration of that alloy can be specified by the vector $\boldsymbol{\sigma}=\left\{\sigma_{1}, \sigma_{2}, \sigma_{3}, \ldots, \sigma_{N}\right\}$. The product of spin variables $\sigma_{i}$ in turn determines a basis function for a given $n$-site cluster $f, \Phi_{f}^{(n)}(\boldsymbol{\sigma})$, given by;

$$
\Phi_{f}^{(n)}(\boldsymbol{\sigma})=\prod_{i \in f} \sigma_{i}
$$

These functions form a complete and orthonormal set with the inner product between two functions, given by;

$$
\left\langle\Phi_{f}^{(n)}(\boldsymbol{\sigma}), \Phi_{g}^{(n)}(\boldsymbol{\sigma})\right\rangle=\frac{1}{2^{n}} \sum_{\boldsymbol{\sigma}} \Phi_{f}^{(n)}(\boldsymbol{\sigma}) \Phi_{g}^{(n)}(\boldsymbol{\sigma})=\delta_{f, g} .
$$

The sum in Eq.(4.2) runs over all atomic configuration $\boldsymbol{\sigma}$, and $\delta_{f, g}$ is the Kronecker's delta. The scalar product, obtained from Eq.(4.2), is thus equal to 1 only if $\Phi_{f}^{(n)}(\boldsymbol{\sigma})$ and $\Phi_{g}^{(n)}(\boldsymbol{\sigma})$ are specifying exactly the same $n$-site clusters in the crystal, while the product of any two clusters differing by at least one atomic sites is always equal to 0 .

As the basis set is complete and orthonormal, one can expand any alloy property $F(\boldsymbol{\sigma})$, which is a function of the configuration in this basis set;

$$
F(\boldsymbol{\sigma})=\sum_{f} F_{f}^{(n)} \Phi_{f}^{(n)}(\boldsymbol{\sigma}),
$$

in which the sum is running over all clusters in the considered alloy system. The expansion coefficients $F_{f}^{(n)}$ are obtained from the projections of $F(\boldsymbol{\sigma})$ on the basis functions $\Phi_{f}^{(n)}(\boldsymbol{\sigma})$;

$$
F_{f}^{(n)}=\left\langle F(\boldsymbol{\sigma}), \Phi_{f}^{(n)}(\boldsymbol{\sigma})\right\rangle .
$$

As for the configurational energy $E_{\text {conf }}(\boldsymbol{\sigma})$, the coefficients are so-called the $n$-site configurational interactions, given by;

$$
V_{f}^{(n)}=\left\langle E_{\text {conf }}(\boldsymbol{\sigma}), \Phi_{f}^{(n)}(\boldsymbol{\sigma})\right\rangle .
$$

Due to the symmetry of the crystal, one can define the $n$-site correlation function $\xi_{\alpha}^{(n)}$ for a particular geometrical figure $\alpha$, often also referred to as cluster, as the average value of the symmetrically equivalent $\Phi_{f}^{(n)}(\boldsymbol{\sigma})$ for a given configuration $\boldsymbol{\sigma}$;

$$
\xi_{\alpha}^{(n)}(\boldsymbol{\sigma})=\left\langle\Phi_{\alpha}^{(n)}\right\rangle=\frac{1}{m_{\alpha}^{(n)}} \sum_{\forall f \in \alpha} \Phi_{f}^{(n)}(\boldsymbol{\sigma}),
$$

where $m_{\alpha}^{(n)}$ is the multiplicity of figure $\alpha$. Consequently, the configurational energy $E_{\text {conf }}(\boldsymbol{\sigma})$ for any atomic configuration $\boldsymbol{\sigma}$ can be expressed by;

$$
E_{\text {conf }}(\boldsymbol{\sigma})=\sum_{\alpha} V_{\alpha}^{(n)} m_{\alpha}^{(n)} \xi_{\alpha}^{(n)}(\boldsymbol{\sigma})
$$




\subsection{Connolly-Williams cluster expansion method}

According to the expression, given in Eq.(4.7), if the interactions $V_{\alpha}^{(n)}$ were known, the configurational energy of any configuration could be easily calculated, or alternatively, one could use them to construct the configurational Hamiltonian $\hat{H}_{\text {conf }}$ for statistical mechanics simulations. For a binary alloy $A_{1-x} B_{x}$, the Hamiltonian is given by;

$$
\hat{H}_{\text {conf }}=\frac{1}{2} \sum_{p} V_{p}^{(2)} \sum_{i, j \in p} \sigma_{i} \sigma_{j}+\frac{1}{3} \sum_{t} V_{t}^{(3)} \sum_{i, j, k \in t} \sigma_{i} \sigma_{j} \sigma_{k}+\cdots,
$$

where $V_{p}^{(2)}$ and $V_{t}^{(3)}$ are the effective pair and three-site interactions for the corresponding pair and three-site clusters, respectively. Unfortunately, in most cases, $V_{\alpha}^{(n)}$ are not known a priori. One way to determine $V_{\alpha}^{(n)}$ in first principles calculations is to use the Connolly-Williams cluster expansion method [153]. The method assumes that Eq.(4.7) could be inverted. That is, if the total energies for a set of predefined configurations $\{\gamma\}$ are known, the interactions $V_{\alpha}^{(n)}$ can be directly determined by solving a set of linear equations;

$$
\mathbf{E}^{\{\gamma\}}=\boldsymbol{\Xi}^{\{\gamma\}} \mathbf{V}
$$

$\mathbf{E}^{\{\gamma\}}$ is the column vector, representing the total energies of the predefined configurations $\{\gamma\}$, which can be calculated from the density functional theory (DFT). $\Xi^{\{\gamma\}}$ is the matrix of correlation functions, whose elements are given by $m_{\alpha}^{(n)} \xi_{\alpha}^{(n)}(\gamma)$, predefined for the configurations $\{\gamma\}$. V is the column vector of the interactions $V_{\alpha}^{(n)}$. Since Eq.(4.9) uses the total energies (or enthalpies at a given pressure) of alloys as input parameters, and local lattice relaxations of the alloys can be taken into account in the calculations of the energies, the method has been widely used to study the phase stability of alloys. Despite its straightforward implementation, the method displays a number of problems and is thus subjected to continuous development by several research groups [155]. The general problem of the method is that one never knows how many interactions $V_{\alpha}^{(n)}$ should be included in the cluster expansion, i.e. Eq.(4.9). Given the fact that the interactions $V_{\alpha}^{(n)}$ are not restricted by any a prior $i$ known information, the total energies $E_{\text {tot }}^{\gamma}$ of the alloys can in principle be identically reproduced by different sets of interactions $V_{\alpha}^{(n)}$.

\subsection{Special quasirandom structure method}

If computational efforts were not a factor, one could construct a huge supercell, that would represent the self-averaging properties of an ideally random alloy, stable in the limit $V / T \rightarrow 0$, where $V$ is the strongest configurational interaction in that particular alloy system and $T$ is the temperature. A practical approximation with reasonable supercell sizes can be achieved using the special quasirandom structure (SQS) approach, suggested by Zunger et al. [41]. In the case of completely random alloys, $\xi_{\alpha}^{(n)}$ are zero for all figures $\alpha$ and they are practically often defined by 
the Warren-Cowley short range order (SRO) parameters that represent the 2site correlation functions [155]. For a binary alloy, $A_{1-x} B_{x}$, the SQS approach constructs a supercell by distributing $A$ - and $B$-type atoms in a way that the SRO parameters between both kinds of atoms are zero, or close to zero for as many coordination shells as possible with focus on the short range shells in order to mimic the configuration, stable in the limit $V / T \rightarrow 0$. Since it is not practically possible, in most cases, to obtain zero value of the SRO parameters for all coordination shells due to the limitation of the supercell size, the configurational energy $E_{\text {conf } f}$, given in Eq.(4.7), does not vanish and could affect predictions of alloys properties. For this reason, the properties - for example, total energy - of the SQS structures need to be converged with respect to the supercell size. In fact real alloys typically display some degree of temperature-dependent clustering and/or ordering at shortrange scales. However, the SQS approach provides an unbiased starting point for modeling substitutionally disordered alloys, for which their configurational entropy can be derived analytically with a random-mimicking configuration (see Chapter 5). Also, the SQS approach can be extended by deliberate creation of supercells with small degrees of SRO, if such cases are of particular interest.

\subsection{Superatom-special quasirandom structure}

Modeling of configurational disorder in icosahedral boron-rich solids, exhibiting complex crystal structures, by implementing the complete mathematical apparatus of Sanchez, Ducastelle, and Gratias [38-40], as described in section 4.1, is often impractical, in particular if a property that is somewhat more complicated to calculate than the total energy is needed - for instance, vibrational free energy and elastic stiffness tensor. As for boron carbide, displaying the large solubility range of carbon, together with the complexity of the icosahedral network, numerous kinds of $\mathrm{B} / \mathrm{C}$ substitutional defects are conceivable. However, it might not be necessary to take into account all of them in modeling configurational disorder in boron carbide, as many of them might be neglected due to high defect formation energies. For this reason, one can try to reveal the important physics of the disordered boron carbide by focusing on modeling of low-energy disordered patterns. It is understandable that configurational disorder in boron carbide, induced by such defects, can be considered as a random distribution of different superatoms, each defined based on knowledge of low-energy dilute defects. This is the basic idea of a superatom-special quasirandom structure (SA-SQS) approach for modeling configurational disorder in boron carbide, arising from a high concentration of low-energy B/C substitutional defects (Paper I). In the SA-SQS approach, the configuration of the defined superatoms is mimicking a random alloy pattern, according to the SQS approach. The following paragraphs describe how the SA-SQS technique can be implemented for modeling configurational disorder in icosahedral boron-rich solids, especially boron carbide, having the compositions of $\mathrm{B}_{4} \mathrm{C}$ and $\mathrm{B}_{13} \mathrm{C}_{2}$.

As for $\mathrm{B}_{4} \mathrm{C}$, whose ground-state configuration is presumably represented by $\mathrm{B}_{11} \mathrm{C}^{p}(\mathrm{CBC})$, in which the icosahedral carbon $\left(\mathrm{C}^{p}\right)$ atoms occupy the same polar 


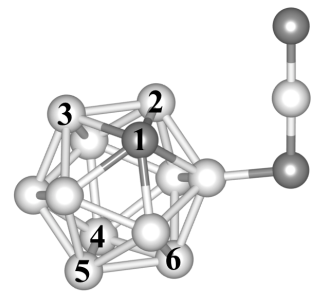

(a)

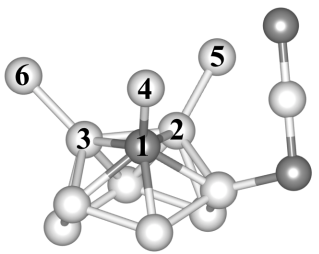

(b)

Figure 4.1. Example of superatomic bases for modeling configurational disorder in boron carbide $\mathrm{B}_{4} \mathrm{C}$ : (a) Basis-1, representing a whole icosahedron and a triatomic chain. (b) Basis-2, whose polar-down sites are replaced by the corresponding polar-down sites of the neighboring icosahedra, bonded to it. White and grey spheres are boron and carbon atoms, respectively. A set of numbers $\{1,2,3\}$ denotes the polar-up sites, while the set $\{4,5,6\}$ denotes the polar-down sites.

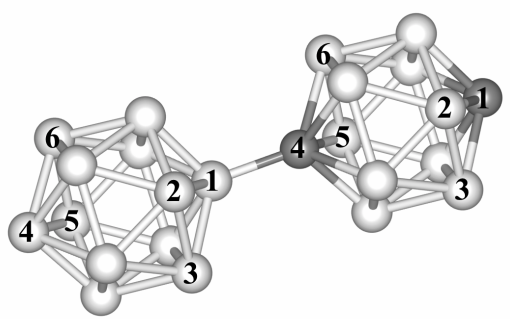

Figure 4.2. A bipolar defect involved with two icosahedra, i.e. $\mathrm{B}_{10} \mathrm{C}_{2}^{p}$ and $\mathrm{B}_{12}$. Numbers and colors denote, respectively, the same crystallographic sites and types of atoms, as described in the caption of Fig.4.1. The triatomic atom chains are not shown.

positions for every icosahedron [56-58, 60, 73], there are two types of dilute B/C substitutional defects that are found to have distinctly lowest formation energy (Paper I). The first kind of low-energy defects originates from the site displacement of the $\mathrm{C}^{p}$ atom among the three polar-up sites, as labeled in Fig.4.1(a) by the numbers 1,2 , and 3 , whiles the second is known as a bipolar defect, originating from the substitution of the $\mathrm{C}^{p}$ atom from one icosahedron for the $\mathrm{B}^{p}$ atom from a neighboring icosahedron forming $\mathrm{B}_{10} \mathrm{C}_{2}^{p}$ and $\mathrm{B}_{12}$, as depicted in Fig.4.2. According to the calculations, performed in this thesis, the defect formation energies of two such low-energy defects are about $0.2 \mathrm{eV}$, whiles other dilute $\mathrm{B} / \mathrm{C}$ substitutional defects, e.g. $\mathrm{B}_{11} \mathrm{C}^{e}(\mathrm{CBC}), \mathrm{B}_{11} \mathrm{C}^{p}(\mathrm{BCC}), \mathrm{B}_{12}(\mathrm{CCC})$, display at least twice as high in the formation energy, ranging from $0.4 \mathrm{eV}$ to $2.6 \mathrm{eV}$.

The first intuitive way to define the superatom basis is to identify the basic structural unit of boron carbide, composing of an icosahedron and a triatomic chain, as the superatom basis, namely Basis-1 as shown in Fig.4.1(a). The superatomic species can be distinguished by the respective positions of the $\mathrm{C}^{p}$ atoms. An 


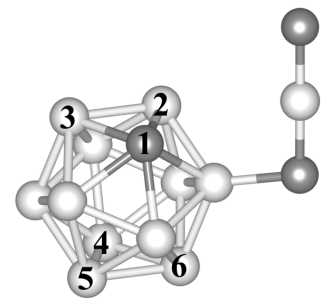

(a)

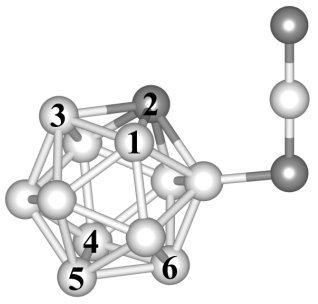

(b)

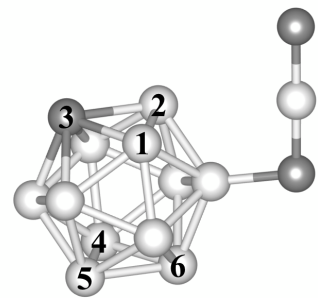

(c)

Figure 4.3. Example of three superatomic species in Basis-1: (a) $B_{11} C^{p(1)}(C B C)$, (b) $\mathrm{B}_{11} \mathrm{C}^{p(2)}(\mathrm{CBC})$, and (c) $\mathrm{B}_{11} \mathrm{C}^{p(3)}(\mathrm{CBC})$, where the numbers 1, 2, and 3 in the parentheses denote the positions of the $\mathrm{C}^{p}$ atoms, corresponding to the notations labeled in the figures. White and grey spheres are boron and carbon atoms, respectively.

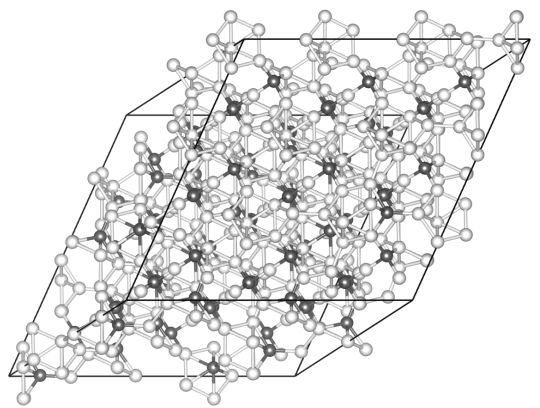

(a)

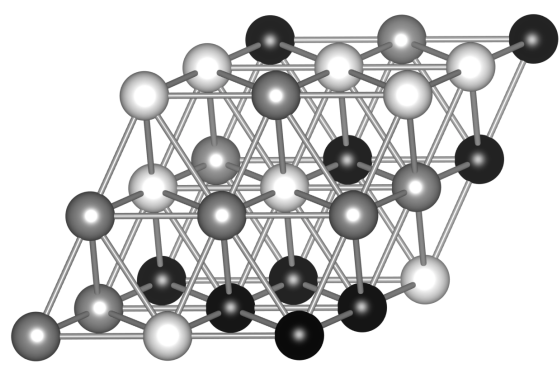

(b)

Figure 4.4. Example of configurationally disordered $\mathrm{B}_{4} \mathrm{C}$ in a $3 \times 3 \times 3$ supercell, represented in (a) normal-atom picture, and (b) superatom picture. The supercell consists of three superatomic species: $\mathrm{B}_{11} \mathrm{C}^{p(1)}(\mathrm{CBC}), \mathrm{B}_{11} \mathrm{C}^{p(2)}(\mathrm{CBC})$, and $\mathrm{B}_{11} \mathrm{C}^{p(3)}(\mathrm{CBC})$, illustrated in Fig.4.3. White and grey spheres in (a) stand for boron and carbon atoms, respectively, whiles black, white, and grey spheres in (b) represent, respectively, the three superatomic species, $\mathrm{B}_{11} \mathrm{C}^{p(1)}(\mathrm{CBC}), \mathrm{B}_{11} \mathrm{C}^{p(2)}(\mathrm{CBC})$, and $\mathrm{B}_{11} \mathrm{C}^{p(3)}(\mathrm{CBC})$.

example is given by three superatomic species, $\mathrm{B}_{11} \mathrm{C}^{p(1)}(\mathrm{CBC}), \mathrm{B}_{11} \mathrm{C}^{p(2)}(\mathrm{CBC})$, and $\mathrm{B}_{11} \mathrm{C}^{p(3)}(\mathrm{CBC})$, as illustrated in Fig.4.3. Simply, three such species of superatoms allow one to model a disordered configuration of $\mathrm{B}_{4} \mathrm{C}$ in which the $\mathrm{C}^{p}$ atom, residing in each icosahedron, can occupy any of the three polar-up sites, which is the first kind of low-energy $\mathrm{B} / \mathrm{C}$ defects. To model a disordered configuration of the defined superatoms, one implements the SQS technique to randomly distribute them in a supercell in a way that mimics the configuration of the random alloy. In a maximum entropy procedure, one assigns an equal concentration for each superatomic species. Fig.4.4 illustrates an example of a configurationally 
disordered $\mathrm{B}_{4} \mathrm{C}$, obtained from the SA-SQS approach, where the $\mathrm{C}^{p}$ atom in each icosahedron can occupy any of the three polar-up sites. Together with the three previously defined superatomic species, one can define three more superatomic species, those are $\mathrm{B}_{11} \mathrm{C}^{p(4)}(\mathrm{CBC}), \mathrm{B}_{11} \mathrm{C}^{p(5)}(\mathrm{CBC})$, and $\mathrm{B}_{11} \mathrm{C}^{p(6)}(\mathrm{CBC})$, and include them in modeling configurational disorder in $\mathrm{B}_{4} \mathrm{C}$, where in this case the $\mathrm{C}^{p}$ atoms configurationally disorder at all six polar sites, both up and down.

Basis-1 alone is however not sufficient to account for the bipolar defects. This is because the bipolar defect is not only the coexistence of $\mathrm{B}_{10} \mathrm{C}_{2}^{p}$ and $\mathrm{B}_{12}$ icosahedra, but their particular arrangements need to be taken into consideration. Therefore, in order to allow for the description of both the displacement of the $\mathrm{C}^{p}$ atoms at the polar sites as well as the bipolar defects, one can define a new superatom basis, called Basis-2, as shown in Fig.4.1(b). That is, the three polar-down sites from a single icosahedron, as labeled by the numbers 4, 5, and 6 in Fig.4.1(a), are replaced by the corresponding polar-down sites of its neighboring icosahedra, bonded to the original polar-up sites, and denoted by the numbers 4,5 , and 6 in Fig.4.1(b). Configurational disorder in $\mathrm{B}_{4} \mathrm{C}$, induced by the bipolar defects at high concentration can be easily modeled using Basis- 2 by which two superatomic species are needed in this case; one with the $\mathrm{C}^{p}$ atom at position 1 , and the other with the $\mathrm{C}^{p}$ atom at position 4, as labeled in Fig.4.1(b). Highly configurationally disordered $\mathrm{B}_{4} \mathrm{C}$, where both types of the identified low-energy defects are taken into account, can also be achieved by including more superatomic species in Basis2 (Paper I).

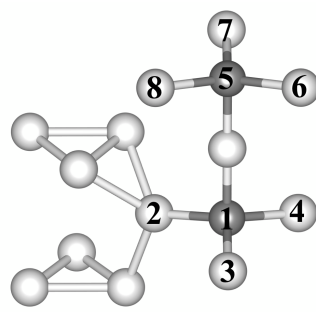

(a)

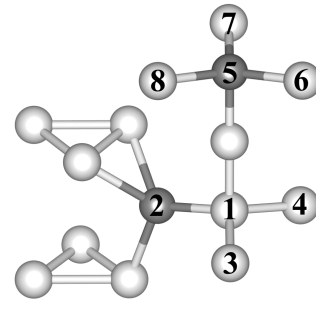

(b)

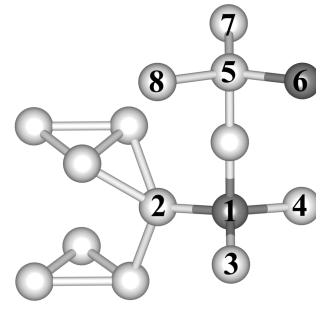

(c)

Figure 4.5. Example of chain-focused superatoms in Basis-3 for modeling configurational disorder in $\mathrm{B}_{13} \mathrm{C}_{2}$. White and grey spheres represent, respectively, boron and carbon atoms. The set of numbers $\{1,5\}$ and $\{2,3,4,6,7,8\}$ denote the chain-end and equatorial sites, respectively, in which (a) stands for a $\mathrm{B}_{12}(\mathrm{CBC})$ superatom, while (b) and (c) represent examples of $\mathrm{B}_{11} \mathrm{C}^{e}(\mathrm{BBC})$ superatoms.

For $\mathrm{B}_{13} \mathrm{C}_{2}$, several theoretical studies $[57,59-61]$ have suggested that its groundstate configuration is given by $\mathrm{B}_{12}(\mathrm{CBC})$, in which the $\mathrm{C}^{p}$ atom in the $\mathrm{B}_{11} \mathrm{C}^{p}(\mathrm{CBC})$ superatom is replaced by a boron atom. The study of $\mathrm{B} / \mathrm{C}$ substitutional defects in the dilute limit (Paper II) indicates that a substitution of a chain-end carbon atom for a neighboring equatorial boron atom, residing in the icosahedron, thus avoiding formation of a $\mathrm{C}-\mathrm{C}$ bond between the triatomic chain and the icosahedron, 
denoted by $\mathrm{B}_{11} \mathrm{C}^{e}(\mathrm{BBC})$, gives the lowest defect formation energy. In this case, the superatomic basis and species must be chosen in a proper way in order to model configurational disorder in $\mathrm{B}_{13} \mathrm{C}_{2}$, which is not only dominated by $\mathrm{B}_{12}(\mathrm{CBC})$ and $\mathrm{B}_{11} \mathrm{C}^{e}(\mathrm{BBC})$ superatomic species, but the substitution of the chain-end carbon atoms for all six equatorial sites are also needed to be considered. Some examples of superatomic species in a new basis, namely Basis-3, for modeling such a disordered configuration of $\mathrm{B}_{13} \mathrm{C}_{2}$, are shown in Fig.4.5. Unlike those in Fig.4.1, Basis-3 is focused on the chain, in which the equatorial sites belonging to a single icosahedron within the basis are replaced by the corresponding equatorial sites of its neighboring icosahedra, labeled by the numbers $2,3,4,6,7$, and 8 in Fig.4.5, bonded to the original intericosahedral chain, corresponding to the numbers 1 , and 5 in the same figure. When defining the superatomic species, one may also assign some constraints in order to avoid the $\mathrm{C}-\mathrm{C}$ bond between the triatomic chain and the icosahedron within a superatom, e.g., the chain-end carbon atoms can swap their positions only with the neighboring equatorial boron atoms bonded to them and only one of the two chain-end atoms is allowed to swap within the single superatom to avoid formation of unfavorable BBB triatomic chain. That is, the chain-end carbon atom at position 1 can swap its position either with the equatorial boron atom at position 2, 3, or 4 (Fig.4.5(b)), while the chain-end carbon atom at position 5 is allowed to swap its position with the equatorial boron atom at position 6,7 , or 8 (Fig.4.5(c)). Consequently, there can be as many as seven species of superatoms considered in modeling configurational disorder in $\mathrm{B}_{13} \mathrm{C}_{2}$, dominated by $\mathrm{B}_{12}(\mathrm{CBC})$ and $\mathrm{B}_{11} \mathrm{C}^{e}(\mathrm{BBC})$ superatoms.

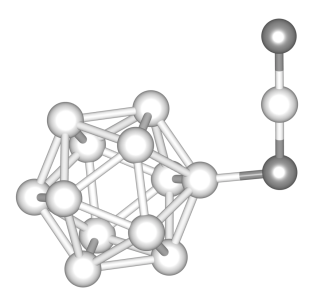

(a)

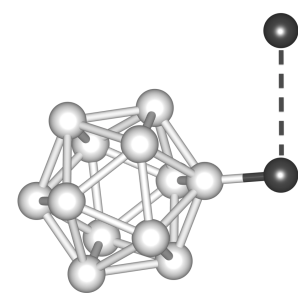

(b)

Figure 4.6. (a) $\mathrm{B}_{12}(\mathrm{CBC})$ and (b) $\mathrm{B}_{12}(\mathrm{O}-\mathrm{O})$ superatoms in Basis-1. White, grey, and black spheres represent boron, carbon, and oxygen atoms, respectively.

Apart from the superatomic bases, the atomic species as well as the numbers of atoms, included in the superatoms, are also changeable. Practically, they can be assigned differently in order to model configurational disorder in other icosahedral boron-rich solids. For instance, if the $\mathrm{CBC}$ chain within the $\mathrm{B}_{12}(\mathrm{CBC})$ superatom, given by Basis-1 (Fig.4.6(a)), is replaced by a pair of isolated oxygen atoms, one obtains $\mathrm{B}_{12}(\mathrm{O}-\mathrm{O})$, which is the basic structural unit of $\mathrm{B}_{6} \mathrm{O}$ (Fig.4.6(b)). One can thus construct theoretical models of $\left(\mathrm{B}_{6} \mathrm{O}\right)_{1-x}\left(\mathrm{~B}_{13} \mathrm{C}_{2}\right)_{x}$ alloys from random distribution of the $\mathrm{B}_{12}(\mathrm{CBC})$ and $\mathrm{B}_{12}(\mathrm{O}-\mathrm{O})$ superatoms in supercells, using the SQS 
technique. In this case, the alloys are only configurationally disordered between superatoms. However, if influences of configurational disorder within superatoms - for example, substitution of either an oxygen or a carbon atom for an icosahedral boron atom - are needed to be taken into account in modeling of the $\left(\mathrm{B}_{6} \mathrm{O}\right)_{1-x}\left(\mathrm{~B}_{13} \mathrm{C}_{2}\right)_{x}$ alloys, a new superatom basis, which can account for their corresponding low-energy substitutional defects must be defined (Paper IV), as done for modeling configurational disorder in $\mathrm{B}_{4} \mathrm{C}$ and $\mathrm{B}_{13} \mathrm{C}_{2}$. The same procedure can also be applied for modeling mixing alloys between $\mathrm{B}_{12} \mathrm{As}_{2}$ and $\mathrm{B}_{12} \mathrm{P}_{2}$ (Paper V), and boron subnitride $\left[\mathrm{B}_{12}(\mathrm{~N}-\mathrm{N})\right]_{0.33}\left[\mathrm{~B}_{12}(\mathrm{NBN})\right]_{0.67}$ (Paper VI), which can be seen as a mixture between $\mathrm{B}_{12}(\mathrm{NBN})$ and $\mathrm{B}_{12}(\mathrm{~N}-\mathrm{N})$ superatoms with a ratio of $2: 1$.

As demonstrated above, the SA-SQS approach can be effectively used for modeling configurational disorder in icosahedral boron-rich solids, especially boron carbide, in which a high concentration of low-energy substitutional defects are distributed in a random manner, following the SQS technique. The stability as well as the properties of the modeled configurationally disordered icosahedral boronrich solids can then be directly investigated, using the density functional theory (DFT), described in Chapter 2.

The SA-SQS approach, however, has also some drawbacks. The technique is particularly designed in order to take into account influences of configurational disorder in icosahedral boron-rich solids. Although the general philosophy might be directly applied to other complex structural solids, considerable reformulations should be needed in its practical implementation, for example, with respect to the definition of superatomic basis. Furthermore, in some cases, in which several superatomic species are needed to be taken into account in modeling configurational disorder, an extremely huge supercell may be needed in order to obtain a reasonably disordered configuration of superatoms, thus resulting in extremely high computational costs.

Additionally, unlike regular atoms, each superatom has an internal structure, which might break the symmetry of the crystal lattice with the consequence of losing the commutative property. Consequently, an averaging procedure between different permutations of superatomic species is understandable, as they not only result in a different internal atomic configuration for each superatomically disordered configuration but also a different total energy, which in turn gives rise to an uncertainty in predicting the properties of a material under consideration, e.g., order-disorder transition temperature. Such an approximation is, however, not problematic in the present study, and the uncertainty in predicting the ordereddisordered transition temperature, caused by this approximation, is shown to be less than 20\% (Paper II). More importantly, the investigations of configurational disorder in boron carbide (Paper I and Paper III) reveal that the intra- and intericosahedral $\mathrm{C}^{p}-\mathrm{C}^{p}$ bonds are very unfavorable types of bonding for boron carbide. However, the implementation of the SA-SQS technique to model configurational disorder in boron carbide - for example, $\mathrm{B}_{4} \mathrm{C}$ using either Basis-1 or Basis-2 (Fig.4.1) - can by chance lead to the formation of the unfavorable $\mathrm{C}^{p}-\mathrm{C}^{p}$ bonds, particularly resulting in high-energy disordered configurations, and thus the orderdisorder transition temperature can be overestimated to a large degree. In reality, 
those $\mathrm{C}^{p}-\mathrm{C}^{p}$ bonds can be avoided by short-range atomic correlations beyond the ideal random configuration, and the entropy loss caused by the avoidance of such bonds is typically small, owing to the low occupancy value of $\mathrm{C}$ atoms at the polar sites in each icosahedron.

It is worth noting that the SA-SQS technique is in fact a further approximation of the normal SQS technique in order to simplify the complexity of modeling configurational disorder in icosahedral boron-rich solids, arising from different sublattices. In the case, where some of the sublattices are configurationally ordered, the SA-SQS and normal SQS techniques can become equivalent. An example is given by modeling of boron subnitride $\left[\mathrm{B}_{12}(\mathrm{~N}-\mathrm{N})\right]_{0.33}\left[\mathrm{~B}_{12}(\mathrm{NBN})\right]_{0.67}($ Paper VI), in which the icosahedra and the chain-end sites are fully occupied boron and nitrogen atoms, respectively. In this case, implementing the SA-SQS technique to model the disordered $\left[\mathrm{B}_{12}(\mathrm{~N}-\mathrm{N})\right]_{0.33}\left[\mathrm{~B}_{12}(\mathrm{NBN})\right]_{0.67}$ alloys by distributing the $\mathrm{B}_{12}(\mathrm{~N}-\mathrm{N})$ and $\mathrm{B}_{12}(\mathrm{NBN})$ superatoms in a random manner, following the SQS technique, is equivalent to the use of the SQS technique to randomly distribute the vacancies "-" and boron atoms at the chain-center sublattice, while the other ordered sublattices are considered as the spectators and do not take part in the SQS modeling. 


\section{CHAPTER 5}

\section{Thermodynamic stability}

It is highly important to know whether a proposed phase of a materials is stable from a thermodynamic point of view. Such analyses can answer the question of under which conditions, generally composition, pressure, and temperature, that the phase will be possible to synthesize under equilibrium, and when instead the relevant competing phases will appear. In principle, the thermodynamic stability of any material with a constant number of particles can be determined as a function of pressure $p$ and temperature $T$ by a physical quantity, the Gibbs free energy $G$;

$$
G(T, p)=E-T S+p V
$$

where $E$ is the total energy of the material, $V$ is the volume, and $S$ is the entropy. At a given $p$ and $T$, the material under consideration will be in thermodynamic equilibrium, when $G$ is globally minimized. The terms $E$ and $S$, given in Eq.(5.1), can at least for practical applications and for physical transparency be divided into different temperature-dependent contributions;

$$
\begin{aligned}
G(T, p) & =E^{\prime}(V)+E_{\text {vib }}(T, V)+E_{\text {conf }}(T, V)+E_{\text {elec }}(T, V)+E_{\text {mag }}(T, V) \\
& -T\left[S_{\text {vib }}(T, V)+S_{\text {conf }}(T, V)+S_{\text {elec }}(T, V)+S_{\text {mag }}(T, V)\right]+p V,
\end{aligned}
$$

where $E^{\prime}(V)$ is the internal ground-state energy of the material. The subscripts vib, conf, elec, and mag denote the contributions to the total energy $E$ and the entropy $S$, arising from the lattice vibrations, configurational disorder, electronic and magnetic excitations, respectively. It is worth noting that the icosahedral boron-rich solids as well as their relevant competing phases, considered in this thesis, are all non-magnetic materials and most of them are semiconductors. As a consequence, the magnetic contributions in Eq.(5.2) can be neglected. The free energy contributions from the electronic excitations are minimal for semiconductors 
and even for metals they are of minor importance, as compared to those arising from the lattice vibrations and configurational disorder, and thus are also being neglected in this thesis. The contributions from the lattice vibrations are entirely defined as the vibrational Helmholtz free energy $F_{v i b}(T, V)$;

$$
F_{v i b}(T, V)=E_{v i b}(T, V)-T S_{v i b}(T, V),
$$

which can be obtained from the quasi-harmonic approximation to the force constants (see Chapter 3). The expression of $F_{v i b}(T, V)$ is given by Eq.(3.20). The configurational part can be obtained in details from a cluster expansion study in combination with Monte Carlo simulations and thermodynamic integration or, conveniently, with the mean-field approximation. As for the latter, one can assume that an ordered phase, for example, ordered compounds and alloys, has the $T=0$ $\mathrm{K}$ properties, whiles a disordered phase, such as random substitutional alloys, has the $T \rightarrow \infty$ properties, that is completely random. For this reason, the Gibbs free energy of the ordered phase $G^{\text {ord }}(T, p)$ can be approximated to be;

$$
G^{o r d}(T, p)=E_{0}^{o r d}(V)+F_{v i b}(T, V)+p V,
$$

where $E_{0}^{\text {ord }}(V)$ is the total energy at $T=0 \mathrm{~K}$ of the ordered phase, calculated from the DFT calculations. Note that $S_{\text {conf }}$ generally vanishes for ordered compounds and alloys. The Gibbs free energy of the disordered phase $G^{d i s}(T, p)$, on the other hand, can be written by;

$$
G^{d i s}(T, p)=E_{0}^{d i s}(V)+F_{v i b}(T, V)-T S_{c o n f}+p V,
$$

where $E_{0}^{\text {dis }}(V)$ is the DFT total energy of the disordered phase with a randommimicking configuration, obtained from the special quasirandom structure (SQS) method (See Chapter 4). Due to the use of the mean-field approach, the configurational entropy $S_{\text {conf }}$ can be analytically derived and is volume- and temperature independent;

$$
S_{\text {conf }}=k_{B} \ln (g),
$$

where $g$ is the number of distinguishable ways of arranging the atoms on the lattice sites, and $k_{B}$ is Boltzmann constant. For a random alloy in the thermodynamic limit, $S_{\text {conf }}$ can be further simplified using Stirling's approximation to be;

$$
S_{\text {conf }}=-k_{B} N \sum_{i} x_{i} \ln \left(x_{i}\right),
$$

where $N$ and $x_{i}$ are defined as the number of lattice sites, in which the mixing is done, and the concentration of the $i^{\text {th }}$ component of the alloy, involved with the configurational disorder, respectively. It is worth noting that, within the superatom framework for modeling configurational disorder in icosahedral boron-rich solids, $N$ and $x_{i}$ are defined, instead, as the number of superatomic sites in a supercell and the concentration of the $i^{\text {th }}$ superatomic species, respectively. Note also that, for a given temperature $T$, the pressure $p$ can be obtained from;

$$
p=-\left[\frac{\partial\left(E_{0}(V)+F_{v i b}(T, V)-T S_{c o n f}\right)}{\partial V}\right]_{T} .
$$




\subsection{Convex hull and formation energy}

The phase stability of a multicomponent material at any specific temperature $T$ and pressure $p$ can be evaluated by creating a plot between the Gibbs free energy $G$ at all considered compositions. That is to search for the stable configurations, determined by minimization of $G$ by allowing for phase decomposition into different fixed compositions. By drawing a set of tie lines, connecting $G$ for every studied compositions, one constructs a so-called convex hull. In order for a material to be stable, it needs to be favorable with respect to any possible decomposition into a set of relevant competing phases, which is in fact a linear optimization problem. The Gibbs free energy of formation $\Delta G$ with respect to constituent elements and/or compounds is generally used to visualize the relevant energy scale, when plotting a convex hull. For example, the stability of a binary compound $A_{m} B_{n}$ with respect to competing pure elements $A$ and $B$ at a given $T$ and $p$ is determined by;

$$
\Delta G\left(A_{m} B_{n}\right)=\frac{G\left(A_{m} B_{n}\right)-(n \cdot G(A))-(m \cdot G(B))}{m+n} .
$$

$\Delta G\left(A_{m} B_{n}\right)$ is the Gibbs free energy of formation/atom for the compound $A_{m} B_{n}$. The term $G\left(A_{m} B_{n}\right)$ is the Gibbs free energy/formula unit of the compound, whiles $G(A)$ and $G(B)$ are the Gibbs free energies/atom for the constituent elements $A$ and $B$, respectively. The considered compound is said to be stable with respect to its constituent elements at that particular temperature $T$ and pressure $p$, if $\Delta G$ is negative, but all compositions need to be considered in order to judge the stability for any particular composition.

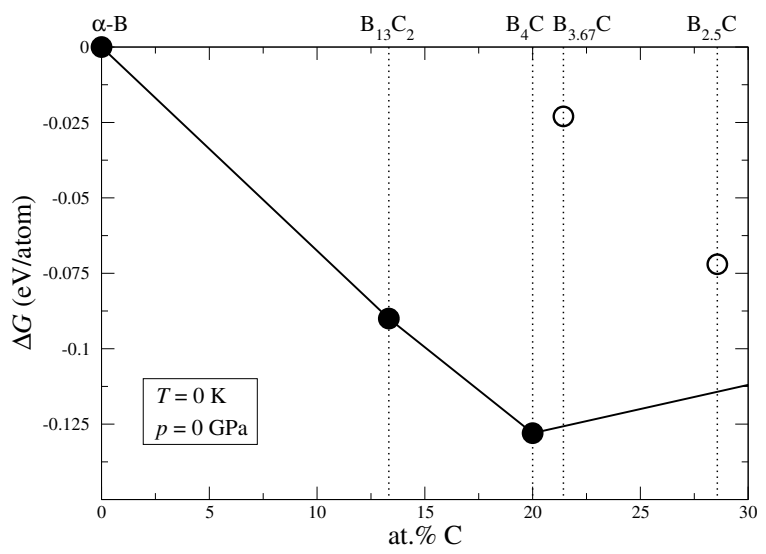

Figure 5.1. Gibbs free energy of formation $\Delta G$ at $T=0 \mathrm{~K}$ and $p=0 \mathrm{GPa}$ of ordered $\mathrm{B}_{13} \mathrm{C}_{2}, \mathrm{~B}_{4} \mathrm{C}, \mathrm{B}_{3.67} \mathrm{C}$, and $\mathrm{B}_{2.5} \mathrm{C}$, calculated with respect to $\alpha$-rhombohedral boron and diamond. The influence of the zero-point motion, i.e. $E_{v i b}(T=0)$, is neglected. The basic structural units of $\mathrm{B}_{13} \mathrm{C}_{2}, \mathrm{~B}_{4} \mathrm{C}, \mathrm{B}_{3.67} \mathrm{C}$, and $\mathrm{B}_{2.5} \mathrm{C}$ are given by $\mathrm{B}_{12}(\mathrm{CBC}), \mathrm{B}_{11} \mathrm{C}^{p}(\mathrm{CBC})$, $\mathrm{B}_{11} \mathrm{C}^{p}(\mathrm{CC})$, and $\mathrm{B}_{10} \mathrm{C}_{2}^{p}(\mathrm{CC})$, respectively. Filled and open circles represent the boron carbides, which are lying on and above the convex hull, respectively. The set of solid lines, connecting the filled circles, indicate the convex hull. 
As for boron carbide, first principles calculations [56-58, 60, 61, 73] predict two stable compositions at $T=0 \mathrm{~K}$ and $p=0 \mathrm{GPa}$, i.e. $\mathrm{B}_{13} \mathrm{C}_{2}$ and $\mathrm{B}_{4} \mathrm{C}$, whose basic structural units are represented by $\mathrm{B}_{12}(\mathrm{CBC})$ and $\mathrm{B}_{11} \mathrm{C}^{p}(\mathrm{CBC})$, respectively. Fig.5.1 illustrates the convex hull of boron carbide, in which both $\mathrm{B}_{12}(\mathrm{CBC})$ and $\mathrm{B}_{11} \mathrm{C}^{p}(\mathrm{CBC})$ are stable with respect to their relevant competing phases, thus lying on the convex hull. Even though $\mathrm{B}_{11} \mathrm{C}^{p}(\mathrm{CC})$ and $\mathrm{B}_{10} \mathrm{C}_{2}^{p}(\mathrm{CC})$, corresponding to $\mathrm{B}_{3.67} \mathrm{C}$ and $\mathrm{B}_{2.5} \mathrm{C}$ compositions [82, 83], respectively, are stable against phase decomposition into $\alpha$-rhombohedral boron and diamond, as indicated by the negative $\Delta G$ shown in Fig.5.1, they are not stable with respect to $\mathrm{B}_{4} \mathrm{C}$ and diamond, thus lying above the convex hull. Note that in fact at $T=0 \mathrm{~K}$ and $p=0 \mathrm{GPa}$ $\beta$-rhombohedral boron and graphite should have been considered as the competing phases of boron carbides. However, due to the ambiguity in the crystal structure of $\beta$-rhombohedral boron [11-13], and van de Waals interactions between layers of graphite that cannot be accurately accounted for by standard DFT calculations, it is more straightforward to consider $\alpha$-rhombohedral boron and diamond instead. Additionally, it is expectable that considering $\alpha$-rhombohedral boron and diamond as the competing phases instead of $\beta$-rhombohedral boron and graphite would not result in a huge difference in determining the stability of boron carbide, since it is shown in the literature that the energy difference between diamond and graphite [156], and between $\alpha$ - and $\beta$-boron [12], are particularly small.

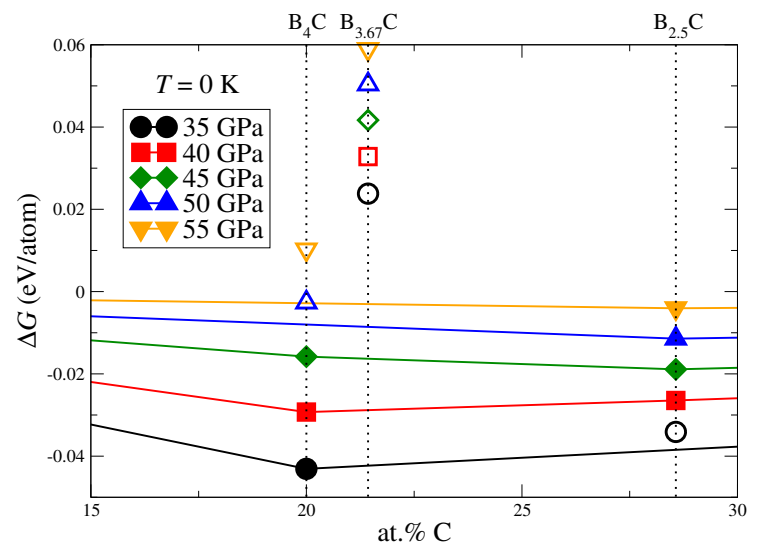

Figure 5.2. Gibbs free energy of formation $\Delta G$ at $T=0 \mathrm{~K}$, calculated with respect to $\gamma$-boron and diamond of $\mathrm{B}_{4} \mathrm{C}, \mathrm{B}_{3.67} \mathrm{C}$, and $\mathrm{B}_{2.5} \mathrm{C}$ under high pressures, ranging from 35 to $55 \mathrm{GPa}$. The influence of the zero-point motion is neglected. Filled and open symbols represent the boron carbides, which are lying on and above the convex hulls at different fixed pressures, respectively. Adapted from Fig.2 in Paper III.

Despite being thermodynamically unstable with respect to $\mathrm{B}_{4} \mathrm{C}$ and diamond at $p=0 \mathrm{GPa}, \mathrm{B}_{2.5} \mathrm{C}$ lies on the convex hull above $40 \mathrm{GPa}$, and is stable up to $57 \mathrm{GPa}$ with respect to both $\mathrm{B}_{4} \mathrm{C}$ and its constituent elements, given by $\gamma$-boron, which is a high-pressure phase of boron, and diamond, as shown by Fig.5.2 (Paper III). The thermodynamic stability of $\mathrm{B}_{2.5} \mathrm{C}$ investigated in this thesis, when 
comparable, is in line with the recent $T=0 \mathrm{~K}$ theoretical study of boron carbides, reported by Jay [83]. $\mathrm{B}_{4} \mathrm{C}$ becomes unstable with respect to $\mathrm{B}_{2.5} \mathrm{C}$ and its constituent elements at $p>47 \mathrm{GPa}$, whiles $\mathrm{B}_{3.67} \mathrm{C}$ never touches the convex hull upon increasing the applied pressure.

By determining the Gibbs free energy of formation $\Delta G$ at different fixed temperatures and pressures of a material with a fixed composition with respect to its competing phases, one can sketch a diagram, illustrating the thermodynamic stability of the material, point-by-point throughout the $p$ - $T$ space. Such a diagram is a so-called phase diagram. An example is given by Fig.5.3, displaying the $p$ - $T$ phase diagram at the global composition of $\mathrm{B}_{2.5} \mathrm{C}$ (Paper III). The phase diagram demonstrates the stability range of the $\mathrm{B}_{2.5} \mathrm{C}$ phase. Under high pressures, ranging between 40 and $67 \mathrm{GPa}$, depending on the temperature, $\mathrm{B}_{2.5} \mathrm{C}$ is thermodynamically stable with respect to $\mathrm{B}_{4} \mathrm{C}, \gamma$-boron and diamond. Below 40 $\mathrm{GPa}, \mathrm{B}_{2.5} \mathrm{C}$ is unstable with respect to $\mathrm{B}_{4} \mathrm{C}$ and diamond, which is in line with the experimental observations that synthesizing boron carbide with at.\% $\mathrm{C} \gtrsim 20$ generally results in a mixture of boron carbide and free carbon $[36,79,80]$. The stability range of $\mathrm{B}_{2.5} \mathrm{C}$ under very high pressure is limited, as it decomposes into $\gamma$-boron and diamond. The prefixes Ordered and Disordered denote the configurations of icosahedral carbon $\mathrm{C}^{p}$ atoms. At $T \gtrsim 500 \mathrm{~K}$, the $\mathrm{C}^{p}$ atoms, residing in the $\mathrm{B}_{10} \mathrm{C}_{2}^{p}(\mathrm{CC})$ units, configurationally disorder at the polar sites, whiles at low temperature, the $\mathrm{C}^{p}$ atoms are well ordered, occupying the same polar positions for every icosahedron. Similarly, the $\mathrm{C}^{p}$ atoms, residing the $\mathrm{B}_{11} \mathrm{C}^{p}(\mathrm{CBC})$ units for the $\mathrm{B}_{4} \mathrm{C}$ composition configurationally disorder at the polar sites at $T \gtrsim 730 \mathrm{~K}$. The same configurational phase transition in $\mathrm{B}_{4} \mathrm{C}$ was also recently reported in the literature [157], in which the order-disorder transition temperature was predicted to be $717 \mathrm{~K}$.

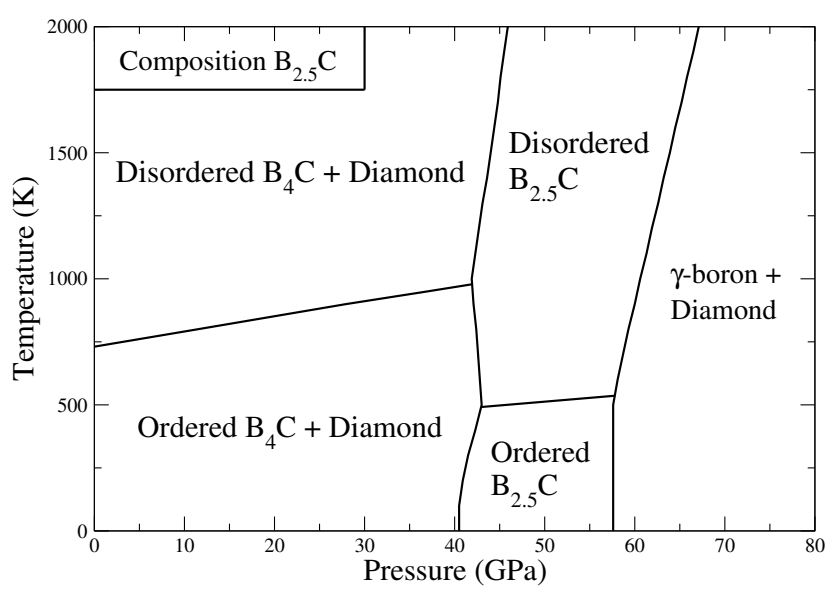

Figure 5.3. $p$ - $T$ phase diagram at the composition of $\mathrm{B}_{2.5} \mathrm{C}$. Adapted from Fig.6(a) in Paper III. The diagram illustrates the existence of both single- and two-phase regions.

In this thesis, apart from the $p$ - $T$ phase diagram at the composition of $\mathrm{B}_{2.5} \mathrm{C}$, shown in Fig.5.3 as the example, the $p$ - $T$ phases diagram at the $\mathrm{B}_{4} \mathrm{C}$ composition 
and at the $\mathrm{B}_{38} \mathrm{~N}_{6}$ composition, illustrating the stability range of boron subnitride $\left[\mathrm{B}_{12}(\mathrm{~N}-\mathrm{N})\right]_{0.33}\left[\mathrm{~B}_{12}(\mathrm{NBN})\right]_{0.67}$, are also sketched, as can be seen from Fig.6(b) in Paper III and from Fig.7 in Paper VI, respectively.

\subsection{Estimation of an order-disorder transition tem- perature in an alloy at a given composition}

For metal alloys, the configurational degree of freedom is often of critical importance for understanding the stability and properties. For a given global composition, there exists a number of way in which the atoms in a single or a combination of several solid solutions can be arranged, giving rise to the configurational contributions to the Gibbs free energy $G$ at $T \geqslant 0 \mathrm{~K}$. As discussed at the beginning of Chapter 5, apart from the contributions from the configurational disorder, other contributions to the Gibbs free energy $G$, e.g., the lattice vibrations, are also of importance. For simplicity, one may consider only the configurational part as a starting point to describe a configurational order-disorder transition in an alloy at a given composition. By assuming also that $p=0 \mathrm{GPa}$, the Gibbs free energy $G$, given by Eq.(5.2) is reduced to;

$$
G(T, 0)=E^{\prime}\left(V_{0}\right)+E_{\text {conf }}\left(T, V_{0}\right)-T S_{\text {conf }}\left(T, V_{0}\right),
$$

where $V_{0}$ denotes the equilibrium volume. The term $-T S_{\text {conf }}\left(T, V_{0}\right)$ indicates a tendency of configurational disorder in a material as the temperature increases. Within the mean-field approximation, assuming that the ordered state has the $T$ $=0 \mathrm{~K}$ properties, the Gibbs free energy for the ordered state $G^{\text {ord }}$ can be written by;

$$
G^{o r d}(0,0)=E_{0}^{o r d}\left(V_{0}\right)
$$

while the Gibbs free energy for the disordered state $G^{\text {dis }}$, assuming the $T \rightarrow \infty$ $\mathrm{K}$ properties, is given by;

$$
G^{d i s}(T, 0)=E_{0}^{d i s}\left(V_{0}\right)-T S_{\text {conf }} .
$$

Note that the definitions of $E_{0}^{\text {ord }}$ and $E_{0}^{\text {dis }}$ in the two equations above are identical to the ones, given in Eq.(5.4) and Eq.(5.5), respectively. However, they are, in this case, evaluated at their equilibrium volume $V_{0}$. The configurational entropy $S_{\text {conf }}$, estimated within the mean-field approximation, is given either by Eq.(5.6) or by Eq.(5.7). The disordered state is thermodynamically stable with respect to the ordered state, if $G^{d i s}$ becomes lower than $G^{\text {ord }}$, and the order-disorder transition temperature $T_{\text {trans }}$ at $p=0 \mathrm{GPa}$ can be estimated from;

$$
T_{\text {trans }}=\frac{E_{0}^{d i s}\left(V_{0}\right)-E_{0}^{o r d}\left(V_{0}\right)}{S_{c o n f}} .
$$

Note that the order-disorder transition temperature $T_{\text {trans }}$, calculated from Eq.(5.13), is generally overestimated by 20-30\% [158]. This is attributed to an absence of the short-range order effects in $E_{0}^{\text {dis }}\left(V_{0}\right)$ within the mean-field approximation, which is not fully compensated by the overestimation of $S_{\text {conf }}$. For instance, 
in this thesis, the order-disorder transition temperature of $\mathrm{B}_{4} \mathrm{C}$ is predicted within the mean-field approach, as described above to be $870 \mathrm{~K}$ (Paper I), whiles a recent theoretical study [157] of configurational disorder in $\mathrm{B}_{4} \mathrm{C}$, using the more accurate Monte Carlo simulations, suggested that the order-disorder transition in $\mathrm{B}_{4} \mathrm{C}$ takes place at 717 K. Fig.5.4 illustrates impacts of vibrational contributions on the configurational order-disorder transition temperature of $\mathrm{B}_{4} \mathrm{C}$, indicating that the transition temperature decreases from $870 \mathrm{~K}$ to $830 \mathrm{~K}$ and $730 \mathrm{~K}$, when the harmonic and quasi-harmonic approximations are, respectively, used to estimate the contributions from the lattice dynamics (see Chapter 3). Thus, in spite of neglecting the contributions arising from the lattice vibrations, taking into account merely the configurational entropy $S_{\text {conf }}$ seems to be sufficient to qualitatively describe the tendency of configurational disorder in icosahedral boron-rich solids. Apart from estimating the order-disorder transition temperatures, the mean-field approximation to the configurational entropy $S_{\text {conf }}$ is also used to investigate impacts of configurational disorder on the thermodynamic stability of the icosahedral boron-rich solids as well as of the relevant competing phases, considered in this thesis. Note that, in some cases, e.g. modeling of configurational disorder in $\mathrm{B}_{13} \mathrm{C}_{2}$ (Paper II) and $\left(\mathrm{B}_{6} \mathrm{O}\right)_{1-x}\left(\mathrm{~B}_{13} \mathrm{C}_{2}\right)_{x}$ alloys (Paper IV) using Basis-3 (see Fig.4.5), in which equally substituting the chain-end $\mathrm{C}$ atoms for only one, two, three, or for all six $\mathrm{B}^{e}$ atoms within the $\mathrm{B}_{12}$ icosahedra practically results in the same total energy, it is still important to fully consider the multiplicity of apparently equivalent superatomic species to approximate $S_{\text {conf }}$, even if only a subset of the superatomic basis is used for the SA-SQS modeling of the energy.

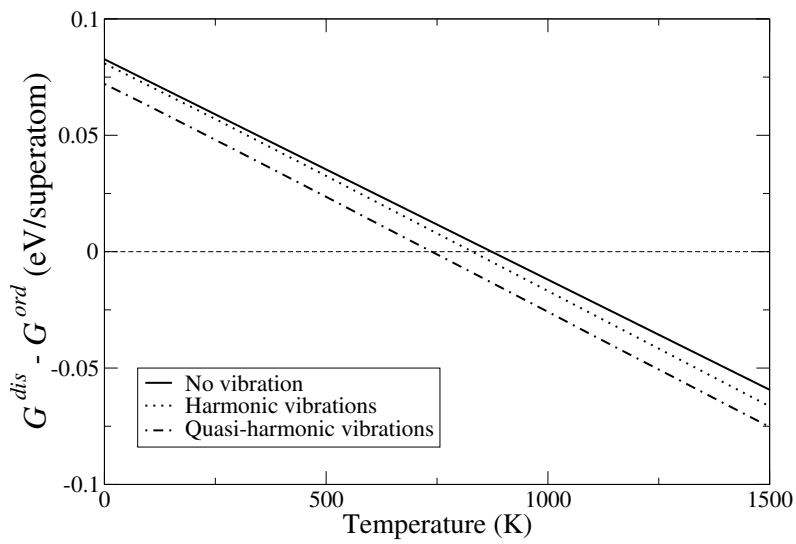

Figure 5.4. Gibbs free energy difference at $p=0 \mathrm{GPa}$ of the disordered $\mathrm{B}_{4} \mathrm{C}$, relative to the ordered $\mathrm{B}_{4} \mathrm{C}$ (dashed line), and plotted as a function of temperature. The configuration of the disordered $\mathrm{B}_{4} \mathrm{C}$ is represented $\mathrm{B}_{11} \mathrm{C}^{p}(\mathrm{CBC})$, in which the $\mathrm{C}^{p}$ atoms configurationally disorder only at the three polar sites, forming the top or bottom triangular face, whiles the $\mathrm{C}^{p}$ atoms of the ordered $\mathrm{B}_{4} \mathrm{C}$ occupy the same polar sites for every icosahedron. The solid line denotes the difference in Gibbs free energy, calculated without taking into account the vibrational contributions. The dotted and dashed-dotted lines represent the differences in Gibbs free energy, where the vibrational contributions are obtained within the harmonic and the quasi-harmonic approximation, respectively. 


\subsection{Mixing thermodynamics of alloys}

As for random substitutional alloys, such as $A_{1-x} B_{x}$ binary alloys, where the composition $x$ can be varied, the thermodynamic stability of the alloys is determined by the mixing Gibbs free energy $\Delta G_{m i x}$, plotted as a function of $x$. The mixing Gibbs free energy $\Delta G_{m i x}$ is given by;

$$
\Delta G_{m i x}(x, T, p)=\Delta H_{m i x}(x, T, p)-T \Delta S_{m i x}(x, T, V) .
$$

$\Delta H_{m i x}$ is the mixing enthalpy, where the enthalpy $H$ is given by;

$$
H(x, T, p)=E_{0}(x, T, V)+p V .
$$

At a given pressure $p$ and composition $x$, the mixing enthalpy $\Delta H_{m i x}$ is defined as the difference in the enthalpy $H$ between the mixing alloy $A_{1-x} B_{x}$ and its constituents $A$ and $B$;

$$
\Delta H_{m i x}=H\left(A_{1-x} B_{x}\right)-[(1-x) \cdot H(A)]-[x \cdot H(B)] .
$$

$\Delta S_{\text {mix }}$ denotes the mixing entropy, describing the difference in the entropy before and after mixing. In this thesis, $\Delta S_{m i x}$ is derived within the mean-field approximation, and can be expressed by Eq.(5.7). For example, $\Delta S_{m i x} /$ atom of the alloy $A_{1-x} B_{x}$ is;

$$
\left.\Delta S_{m i x}=-k_{B}[x \cdot \ln (x)+(1-x) \cdot \ln (1-x))\right] .
$$

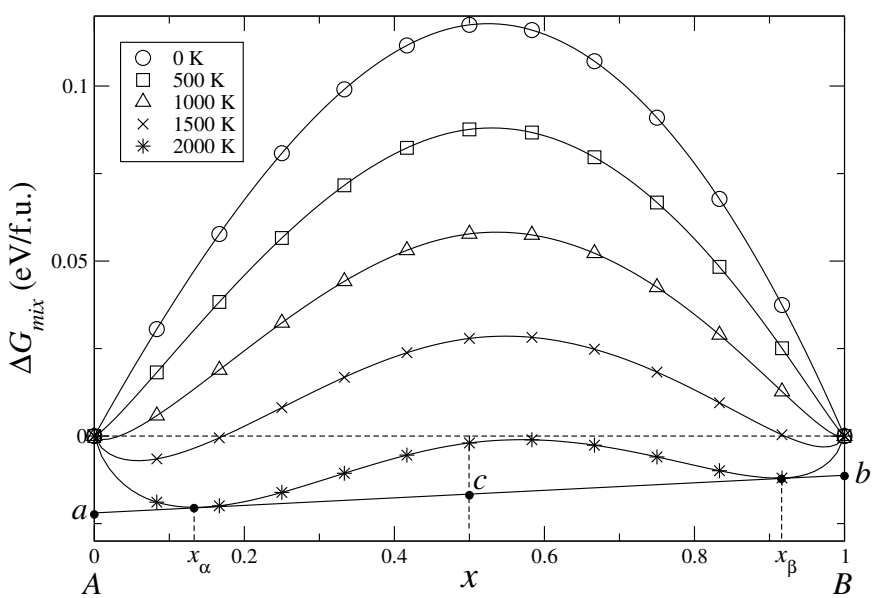

Figure 5.5. $\Delta G_{m i x}$ at a constant pressure of hypothetical alloys $A_{1-x} B_{x}$ as a function of composition $x$ at different temperatures, with respect to their constituents $A$ and $B$ given by the dashed line. A common tangent $\overline{a b}$ is given at $2000 \mathrm{~K}$ as an example.

If $\Delta G_{m i x}<0$, the alloy $A_{1-x} B_{x}$ is stable with respect to its constituents, indicating a tendency of mixing between $A$ and $B$. On the other hand, $\Delta G_{m i x}>$ 0 indicates a tendency of a phase separation. It is worth noting that the stability 
of any alloy at a given composition $x$ cannot be determined only by its positive or negative value of $\Delta G_{m i x}$. In order to determine the stability of the alloy at any fixed composition, $\Delta G_{m i x}$ of the alloy $A_{1-x} B_{x}$ at all compositions $x$ must be considered. This can be done by applying common tangent construction to the $\Delta G_{m i x}$ curves, which will be described in the following paragraphs.

Fig.5.5 illustrates a plot of the mixing Gibbs free energy $\Delta G_{m i x}$ at a constant pressure of hypothetical alloys $A_{1-x} B_{x}$ as a function of the composition $x$ at different absolute temperatures $T$. At $T=0 \mathrm{~K}, \Delta G_{m i x}>0$ for a whole composition range, and the alloys $A_{1-x} B_{x}$ are likely to decompose into $A$ and $B$. As the temperature $T$ increases, the contribution from the mixing entropy $\Delta S_{m i x}$ becomes larger, thus lowering the mixing Gibbs free energy $\Delta G_{m i x}$. In the case, where the $\Delta G_{m i x}$ curves display two minima, $\Delta G_{m i x}$ of the alloy $A_{1-x} B_{x}$ at any composition $x$ between the two minima can be lowered, as it decomposes into two phases. The minimization of $\Delta G_{m i x}$ is determined by a common tangent line, connecting between the two minima. As a consequence, the alloy is most stable, in thermodynamic equilibrium, as a mixture of two phases, in which their compositions are also given by tangential points of the common tangent.

As shown in Fig.5.5, the common tangent line $\overline{a b}$ is drawn at $2000 \mathrm{~K}$ as an example. At that particular temperature, the alloy $A_{1-x} B_{x}$ at any composition $x$ between $x_{\alpha}$ and $x_{\beta}$ can lower its $\Delta G_{m i x}$ by decomposing into two phases $-\alpha$ and $\beta$ for example. Once $\Delta G_{m i x}$ lies above the common tangent $\overline{a b}$, such as point $c$ at $x=0.5$, the alloy is in thermodynamic equilibrium and stable as a mixture of $\alpha$ and $\beta$. The equilibrium compositions of $\alpha$ and $\beta$ phases are given by $x_{\alpha}$ and $x_{\beta}$, respectively, and the relative amounts of the two phases can be obtained by the lever rule. The region where the two phases coexist is known as a miscibility gap. Either for $x<x_{\alpha}$ or for $x>x_{\beta}$, the minimum of the Gibbs free energy already lies on the $\Delta G_{m i x}$ curve, and the alloys $A_{1-x} B_{x}$ within such composition regions are thermodynamically stable as a homogeneous single-phase solid solution. By constructing the common tangents to a set of $\Delta G_{m i x}$ curves at different temperatures, one can sketch a $T-x$ phase diagram [159].

In this thesis, the phase diagrams of pseudo-binary $\left(\mathrm{B}_{6} \mathrm{O}\right)_{1-x}\left(\mathrm{~B}_{13} \mathrm{C}_{2}\right)_{x}$ (Paper IV), $\mathrm{As}_{1-x} \mathrm{P}_{x}$ (Paper V), and $\mathrm{B}_{12}\left(\mathrm{As}_{1-x} \mathrm{P}_{x}\right)_{2}$ (Paper $\mathrm{V}$ ) are outlined in order to illustrate their thermodynamic stability. For example, Fig.5.6 illustrates $\Delta G_{m i x}$ at $p=0 \mathrm{GPa}$ of $\mathrm{B}_{12}\left(\mathrm{As}_{1-x} \mathrm{P}_{x}\right)_{2}$ disordered alloys as a function of the composition $x$ at different fixed $T$ with respect to $\mathrm{B}_{12} \mathrm{As}_{2}$ and $\mathrm{B}_{12} \mathrm{P}_{2}$ (Fig.5.6(a)) and the $T-x$ phase diagram of $\mathrm{B}_{12}\left(\mathrm{As}_{1-x} \mathrm{P}_{x}\right)_{2}$ (Fig.5.6(b)), sketched by drawing the common tangents to the $\Delta G_{m i x}$ curves in Fig.5.6(a). The phase diagram exhibit a miscibility gap at $T \lesssim 913 \mathrm{~K}$, in which a solid solution of $\mathrm{B}_{12}\left(\mathrm{As}_{1-x} \mathrm{P}_{x}\right)_{2}$, denoted by $\alpha$, is stable as a mixture of two $\mathrm{B}_{12}\left(\mathrm{As}_{1-x} \mathrm{P}_{x}\right)_{2}$ phases of different compositions $x\left(\alpha^{\prime}+\alpha^{\prime \prime}\right)$. Note that the mean-field approach, used to estimate $\Delta H_{m i x}$ and $\Delta S_{\text {mix }}$, typically overestimates the maximum temperature for the boundary between a single-phase region $(\alpha)$ and a miscibility gap $\left(\alpha^{\prime}+\alpha^{\prime \prime}\right)$, and thus it should be taken as an upper limit. 


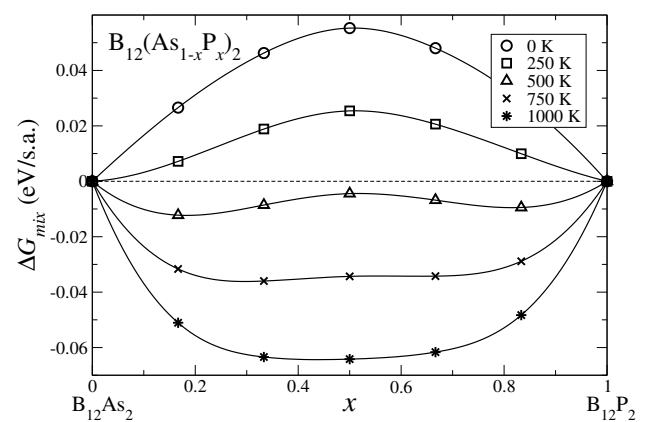

(a)

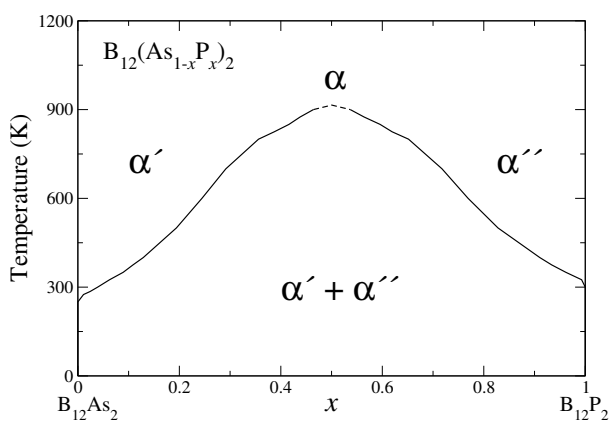

(b)

Figure 5.6. (a) $\Delta G_{m i x}$ at $p=0 \mathrm{GPa}$ of $\mathrm{B}_{12}\left(\mathrm{As}_{1-x} \mathrm{P}_{x}\right)_{2}$ disordered alloys as a function of the composition $x$ at $T=0,250,500,750,1000 \mathrm{~K}$ with respect to $\mathrm{B}_{12} \mathrm{As}_{2}$ and $\mathrm{B}_{12} \mathrm{P}_{2}$. (b) $T$-x phase diagram of $\mathrm{B}_{12}\left(\mathrm{As}_{1-x} \mathrm{P}_{x}\right)_{2}$, obtained from applying the common tangent construction to the $\Delta G_{m i x}$ curves, given in (a). (b) is taken from Fig.7 in Paper V.

\subsection{Thermodynamic stability of ternary compounds and alloys: Linear optimization problem}

Although evaluation of the thermodynamic stability of any material with respect to its competing phases is graphically straightforward, as demonstrated through binary and pseudo-binary systems studied in the previous sections, the situation becomes more complex for ternary and multinary systems. This is because of a large number of relevant competing phases, needed to be taken into consideration in order for construction of the convex hull. In order to search for a combination of the competing phases, that minimizes the Gibbs free energy $G$ at a given composition, temperature $T$, and pressure $p$, one can implement a method, called linear optimization. By following the procedure describe in Ref. [160], the most competitive combination of phases, yielding the minimum Gibbs free energy $G_{m i n}$, for any ternary composition of $x^{A}, x^{B}$, and $x^{C}$ can be found from;

$$
G_{\text {min }}=\min \left\{G\left(x^{A}, x^{B}, x^{C}\right)\right\},
$$

where

$$
G\left(x^{A}, x^{B}, x^{C}\right)=\sum_{i=1}^{n} y_{i} G_{i} .
$$

$y_{i}$ and $G_{i}$ are the amount and Gibbs free energy of the $i^{\text {th }}$ competing phases, respectively. Since the amount $y_{i}$ of any competing phase cannot be negative, and the sum of each element $(A, B$, and $C$ ) from the relevant competing phases must be equal to that of the considered composition, Eq.(5.18) is subjected to the constraints;

$$
y_{i} \geq 0, \quad \sum_{i=1}^{n} y_{i}^{A}=x^{A}, \quad \sum_{i=1}^{n} y_{i}^{B}=x^{B}, \quad \sum_{i=1}^{n} y_{i}^{C}=x^{C} .
$$


The thermodynamic stability of a proposed ternary compound $A_{x^{A}} B_{x^{B}} C_{x^{C}}$, for example, can be determined by the Gibbs free energy of formation $\Delta G$;

$$
\Delta G\left(A_{x^{A}} B_{x^{B}} C_{x^{C}}\right)=G\left(A_{x^{A}} B_{x^{B}} C_{x^{C}}\right)-G_{\min }\left(x^{A}, x^{B}, x^{C}\right) .
$$

If $\Delta G<0$, the compound $A_{x^{A}} B_{x^{B}} C_{x^{C}}$ is thermodynamically stable with respect to the most competitive combination of other phases and thus becomes a part of the convex hull in the ternary $A-B-C$ system. On the other hand, if $\Delta G>0$, the compound is unstable in thermodynamic equilibrium and will thus decompose into the relevant competing phases, yielding the minimum of the Gibbs free energy. By evaluating the thermodynamic stability at a given $T$ and $p$ of all possible phases and compositions in any ternary system, one can outline a ternary phase diagram.

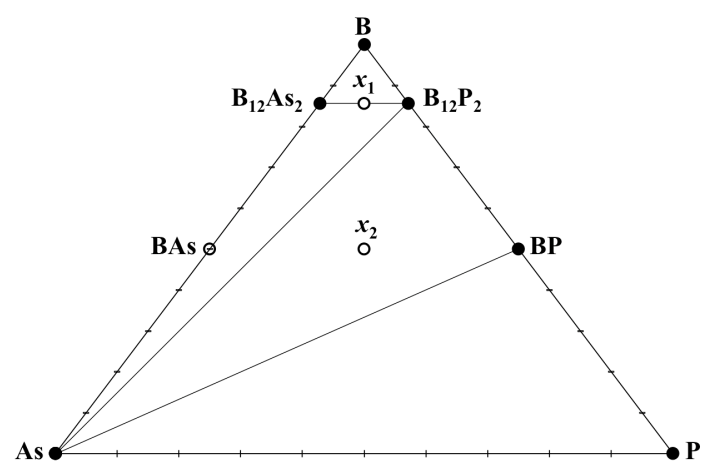

Figure 5.7. Ternary phase diagram of B-As-P at $T=0 \mathrm{~K}$ and $p=0 \mathrm{GPa}$. The filled and open black circles denote the stable, making up the convex hull, and examples of unstable phases, lying above the convex hull, respectively. $x_{1}$ and $x_{2}$ represent icosahedral $\mathrm{B}_{12}\left(\mathrm{As}_{0.5} \mathrm{P}_{0.5}\right)_{2}$ and zinc-blende $\mathrm{BAs}_{0.5} \mathrm{P}_{0.5}$ disordered alloys, respectively. Adapted from Fig.8(a) in Paper V.

In this thesis, the linear optimization method is used to determine the thermodynamic stability of the pseudo binary $\left(\mathrm{B}_{6} \mathrm{O}\right)_{1-x}\left(\mathrm{~B}_{13} \mathrm{C}_{2}\right)_{x}$ alloys (Paper IV) and of different compounds and alloys in the ternary B-As-P system (Paper V). For example, Fig.5.7 illustrates the ternary phase diagram of B-As-P system at $T$ $=0 \mathrm{~K}$ and $p=0 \mathrm{GPa}$. The filled black circles stand for the stable phases, making up the convex hull. Those are, $\alpha$-rhombohedral boron (B), black phosphorus $(\mathrm{P})$, gray arsenic (As), zinc blende boron phosphide (BP), icosahedral boron subarsenide $\left(\mathrm{B}_{12} \mathrm{As}_{2}\right)$ and boron subphosphide $\left(\mathrm{B}_{12} \mathrm{P}_{2}\right)$. The open black circles, on the other hand, denote the unstable phases, lying above the convex hull, e.g., zinc blende boron arsenide (BAs), which is thermodynamically unstable with respect to $\mathrm{B}_{12} \mathrm{As}_{2}$ and pure arsenic phase. The open circle $x_{1}$, shown in Fig.5.7, represents the icosahedral $\mathrm{B}_{12}\left(\mathrm{As}_{0.5} \mathrm{P}_{0.5}\right)_{2}$ disordered alloy, which is thermodynamically unstable with respect to $\mathrm{B}_{12} \mathrm{As}_{2}$ and $\mathrm{B}_{12} \mathrm{P}_{2}$ at $T=0 \mathrm{~K}$. The zinc-blende $\mathrm{BAs}_{0.5} \mathrm{P}_{0.5}$ disordered alloys, denoted by the open circle $x_{2}$ in Fig.5.7, is also unstable and will decompose into $\mathrm{B}_{12} \mathrm{P}_{2}, \mathrm{BP}$, and $\mathrm{As}$ under thermodynamic equilibrium condition at $T=0 \mathrm{~K}$. 
CHAPTER 6

\section{Modeling elastic properties}

Besides the thermodynamic stability and the crystallographic, electronic, and vibrational properties, it is as well of interest to explore the elastic properties of icosahedral boron-rich solids, as some of them, such as boron carbide and boron suboxide, are candidates for being superhard materials. The elastic properties, generally determined by elastic constants, provide an important part of the information on the mechanical behavior, e.g., the mechanical stability and the stiffness, of materials, which is of great importance for technological applications, such as in cutting tools and other cases, where wear resistance is needed. The plastic properties of a material, on the one hand, composes a formidable challenge for atomistic modeling. It is, however, indirectly related to the elastic properties. In this thesis, the elastic properties of boron carbides (Paper III and IV), $\left(\mathrm{B}_{6} \mathrm{O}\right)_{1-x}\left(\mathrm{~B}_{13} \mathrm{C}_{2}\right)_{x}$ alloys (Paper IV), and boron subnitrides (Paper VI) are calculated at $p=0 \mathrm{GPa}$ and $T=0 \mathrm{~K}$, irrespective of the influence of the zero-point motion. Thus, the Gibbs free energy $G$, given in Eq. (5.4), reduces to the total energy $E_{0}$. By applying strains $\epsilon$ with $\pm 1 \%$ and $\pm 2 \%$ distortions to the fully relaxed supercells without volume conservation, the elastic constants $C_{i j}$ can be directly calculated from the second-order Taylor expansion of the total energy $E_{0}$ as a function of the imposed strains $\epsilon[161,162]$;

$$
C_{i j}=\left.\frac{1}{V_{0}} \frac{\partial^{2} E_{0}(\boldsymbol{\epsilon})}{\partial \epsilon_{i} \partial \epsilon_{j}}\right|_{\boldsymbol{\epsilon}=0},
$$

where Voigt's notation is used to describe the strain vector $\epsilon$ and the elastic tensor $C[163,164]$. The strain vector $\epsilon$ is thus given by;

$$
\boldsymbol{\epsilon}=\left\{\epsilon_{1}, \epsilon_{2}, \epsilon_{3}, \epsilon_{4}, \epsilon_{5}, \epsilon_{6}\right\}
$$


$E_{0}(\boldsymbol{\epsilon})$ is the total energy of the supercell, distorted by the correspondingly applied strains $\epsilon_{i}$, and can be expressed in term of a power series of the strains $\epsilon$ by [162];

$$
E_{0}(\boldsymbol{\epsilon})=E_{0}(0)+V_{0}\left(\sum_{i=1}^{6} \tau_{i} \epsilon_{i}+\frac{1}{2} \sum_{i=1}^{6} \sum_{j=1}^{6} C_{i j} \epsilon_{i} \epsilon_{j}+O\left(\epsilon^{3}\right)\right),
$$

where $E_{0}(0)$ and $V_{0}$ are the total energy and equilibrium volume of the undistorted supercell, respectively. $\tau_{i}$ are the components of the stress vector $\tau$, which vanishes if the undistorted supercell is stress free, as the derivatives in Eq.(6.1) are evaluated at $\epsilon=0$.

Practically, one can obtain a strain-induced distorted supercell by applying the strains $\epsilon_{i}$ to the undistorted one via [165];

$$
\left(\begin{array}{l}
\boldsymbol{a}_{1}^{\prime} \\
\boldsymbol{a}_{2}^{\prime} \\
\boldsymbol{a}_{3}^{\prime}
\end{array}\right)=\left(\begin{array}{l}
\boldsymbol{a}_{1} \\
\boldsymbol{a}_{2} \\
\boldsymbol{a}_{3}
\end{array}\right) \cdot(I+\varepsilon)
$$

where $\boldsymbol{a}_{i}^{\prime}$ and $\boldsymbol{a}_{i}(i=1,2,3)$ represent the lattice vectors of the distorted and the undistorted supercells, respectively. $I$ is the unitary matrix, and $\varepsilon$ is the strain tensor, which is related to the strain vector $\epsilon$ in Eq.(6.2) by [165];

$$
\varepsilon=\left(\begin{array}{ccc}
\epsilon_{1} & \frac{\epsilon_{6}}{2} & \frac{\epsilon_{5}}{2} \\
\frac{\epsilon_{6}}{2} & \epsilon_{2} & \frac{\epsilon_{4}}{2} \\
\frac{\epsilon_{5}}{2} & \frac{\epsilon_{4}}{2} & \epsilon_{3}
\end{array}\right)
$$

The second-order elastic stiffness tensor $C$ can be represented by a $6 \times 6$ matrix and has in principle a maximum of 36 independent elements. As the $6 \times 6$ matrix representation of $C$ is symmetric, i.e. $C_{i j}=C_{j i}$, the maximum number of the independent elastic constants $C_{i j}$ reduces to 21 . The elastic tensor $C$ can thus be given by;

$$
C=\left(\begin{array}{llllll}
C_{11} & C_{12} & C_{13} & C_{14} & C_{15} & C_{16} \\
C_{12} & C_{22} & C_{23} & C_{24} & C_{25} & C_{26} \\
C_{13} & C_{23} & C_{33} & C_{34} & C_{35} & C_{36} \\
C_{14} & C_{24} & C_{34} & C_{44} & C_{45} & C_{46} \\
C_{15} & C_{25} & C_{35} & C_{45} & C_{55} & C_{56} \\
C_{16} & C_{26} & C_{36} & C_{46} & C_{56} & C_{66}
\end{array}\right)
$$

The number of independent elements for the elastic tensor $C$ also depends on the crystal symmetry. As for the icosahedral boron-rich solids, considered in this thesis, they all exhibit the rhombohedral symmetry $(R \overline{3} m)$. In this case, the elastic tensor $C_{\text {rhomb }}$ can be represented by six independent elastic constants, i.e. $C_{11}$, $C_{12}, C_{13}, C_{14}, C_{33}$, and $C_{44}$;

$$
C_{r h o m b}=\left(\begin{array}{cccccc}
C_{11} & C_{12} & C_{13} & C_{14} & 0 & 0 \\
C_{12} & C_{11} & C_{13} & -C_{14} & 0 & 0 \\
C_{13} & C_{13} & C_{33} & 0 & 0 & 0 \\
C_{14} & -C_{14} & 0 & C_{44} & 0 & 0 \\
0 & 0 & 0 & 0 & C_{44} & C_{14} \\
0 & 0 & 0 & 0 & C_{14} & C_{66}
\end{array}\right),
$$


where

$$
C_{66}=\frac{1}{2}\left(C_{11}-C_{12}\right)
$$

Since the use of the SQS technique for modeling random alloys, in principle, breaks the point group symmetry, the projection technique of Moakher et al. [166] is implemented in order to derive the rhombohedrally averaged elastic tensors $\bar{C}_{r h o m b}$;

$$
\bar{C}_{r h o m b}=\left(\begin{array}{cccccc}
\bar{C}_{11} & \bar{C}_{12} & \bar{C}_{13} & \bar{C}_{14} & 0 & 0 \\
\bar{C}_{12} & \bar{C}_{11} & \bar{C}_{13} & -\bar{C}_{14} & 0 & 0 \\
\bar{C}_{13} & \bar{C}_{13} & \bar{C}_{33} & 0 & 0 & 0 \\
\bar{C}_{14} & -\bar{C}_{14} & 0 & \bar{C}_{44} & 0 & 0 \\
0 & 0 & 0 & 0 & \bar{C}_{44} & \bar{C}_{14} \\
0 & 0 & 0 & 0 & \bar{C}_{14} & \bar{C}_{66}
\end{array}\right),
$$

for the configurationally disordered icosahedral boron-rich solids, modeled by the SA-SQS approach, where

$$
\begin{array}{r}
\bar{C}_{11}=\frac{3}{8}\left(C_{11}+C_{22}\right)+\frac{1}{4} C_{12}+\frac{1}{2} C_{66}, \\
\bar{C}_{12}=\frac{1}{8}\left(C_{11}+C_{22}\right)+\frac{3}{4} C_{12}-\frac{1}{2} C_{66}, \\
\bar{C}_{13}=\frac{1}{2}\left(C_{13}+C_{23}\right), \\
\bar{C}_{14}=\frac{1}{4} C_{14}-\frac{1}{4} C_{24}+\frac{1}{2} C_{56}, \\
\bar{C}_{33}=C_{33}, \\
\bar{C}_{44}=\frac{1}{2} C_{44}+C_{55}, \\
\bar{C}_{66}=\frac{1}{2}\left(\bar{C}_{11}-\bar{C}_{12}\right)=\frac{1}{8}\left(C_{11}+C_{22}\right)-\frac{1}{4} C_{12}+\frac{1}{2} C_{66} .
\end{array}
$$

Consequently, twelve independent elastic constants, i.e. $C_{11}, C_{12}, C_{13}, C_{14}, C_{22}$, $C_{23}, C_{24}, C_{33}, C_{44}, C_{55}, C_{56}$, and $C_{66}$, are needed in a SA-SQS calculation to obtain $\bar{C}_{r h o m b}$, given in Eq.(6.9). The elastic stability of the icosahedral boron-rich solids is verified by the Born stability criteria [167] for the rhombohedral structure;

$$
\begin{aligned}
\bar{C}_{11}-\left|\bar{C}_{12}\right| & >0, \\
\left(\bar{C}_{11}+\bar{C}_{12}\right) \bar{C}_{33}-2\left(\bar{C}_{13}\right)^{2} & >0, \\
\left(\bar{C}_{11}-\bar{C}_{12}\right) \bar{C}_{44}-2\left(\bar{C}_{14}\right)^{2} & >0, \\
\bar{C}_{44} & >0 .
\end{aligned}
$$

The isotropic elastic moduli and Poisson's ratio of the icosahedral boron-rich solids are obtained using the Voigt-Reuss-Hill (VRH) approach [168] for calculating elastic properties of polycrystalline solids. The Voigt method averages the elastic stiffness $\left(C_{i j}\right)$ over all lattice orientations by assuming that strain is uniform throughout a grain. Two independent isotropic elastic moduli, i.e. bulk modulus $B$, and 
shear modulus $G$, given by the Voigt method, are;

$$
\begin{array}{r}
B_{V}=\frac{\left(C_{11}+C_{22}+C_{33}\right)+2\left(C_{12}+C_{13}+C_{23}\right)}{9}, \\
G_{V}=\frac{\left(C_{11}+C_{22}+C_{33}\right)-\left(C_{12}+C_{13}+C_{23}\right)+3\left(C_{44}+C_{55}+C_{66}\right)}{15} .
\end{array}
$$

The Reuss method averages the elastic compliances $\left(S_{i j}\right)$ by assuming that stress is uniform throughout a grain. The elastic compliance tensor $S$ is obtained by the inverse of the elastic stiffness tensor $C$, thus

$$
S=C^{-1}=\left(\begin{array}{cccccc}
S_{11} & S_{12} & S_{13} & S_{14} & S_{15} & S_{16} \\
S_{12} & S_{22} & S_{23} & S_{24} & S_{25} & S_{26} \\
S_{13} & S_{23} & S_{33} & S_{34} & S_{35} & S_{36} \\
S_{14} & S_{24} & S_{34} & S_{44} & S_{45} & S_{46} \\
S_{15} & S_{25} & S_{35} & S_{45} & S_{55} & S_{56} \\
S_{16} & S_{26} & S_{36} & S_{46} & S_{56} & S_{66}
\end{array}\right)
$$

and the two independent elastic moduli within the Reuss method can be obtained as;

$$
\begin{gathered}
B_{R}=\frac{1}{\left(S_{11}+S_{22}+S_{33}\right)+2\left(S_{12}+S_{13}+S_{23}\right)}, \\
G_{R}=\frac{15}{4\left(S_{11}+S_{22}+S_{33}\right)-4\left(S_{12}+S_{13}+S_{23}\right)+3\left(S_{44}+S_{55}+S_{66}\right)} .
\end{gathered}
$$

In this thesis, the Voigt average elastic moduli, $B_{V}$, and $G_{V}$, for the icosahedral boron-rich solids are obtained via Eq.(6.12), by which the elastic constants $C_{i j}$ in the equations are replaced by the rhombohedrally averaged elastic constants $\bar{C}_{i j}$, as determined by Eq.(6.10), while the Reuss average elastic moduli, $B_{R}$, and $G_{R}$, are obtained by replacing the elastic compliances $S_{i j}$ in Eq.(6.14) with the rhombohedrally averaged elastic compliances $\bar{S}_{i j}$, which are the elements of the compliance tensor $\bar{S}_{r h o m b}$. By using the relation between $C$ and $S$, given in Eq.(6.13), the tensor $\bar{S}_{r h o m b}$ can be directly obtained by the inverse of $\bar{C}_{r h o m b}$;

$$
\bar{S}_{r h o m b}=\left(\bar{C}_{r h o m b}\right)^{-1}
$$

Generally, the Voigt average gives the upper bound of elastic moduli, while the Reuss average gives the lower bound of elastic moduli. The Hill average is thus simply the arithmetic average of those obtained from the Voigt and Reuss averages;

$$
\begin{aligned}
B_{H} & =\frac{B_{V}+B_{R}}{2}, \\
G_{H} & =\frac{G_{V}+G_{R}}{2} .
\end{aligned}
$$


The Young's modulus $E$ and the Poisson's ratio $\nu$ for each average method can be obtained via the relationships between the different elastic moduli;

$$
E=\frac{9 B G}{3 B+G}
$$

and

$$
\nu=\frac{3 B-2 G}{2(3 B+G)}
$$




\section{CHAPTER 7}

\section{Conclusions}

As mentioned in Chapter 1, the main objective of this thesis is to address the general questions, related to configurational disorder in icosahedral boron-rich solids, using first-principles methods. Influences of configurational disorder on the stabilities and properties of icosahedral boron-rich solids, in particular boron carbide, is studied. Furthermore, boron subnitride and the mixing thermodynamics between different icosahedral boron-rich solids, those are boron carbide, boron suboxide, and boron subpnictides, are investigated. The studies include also a theoretical method development for modeling configurational disorder in icosahedral boronrich solids. This chapter provides a summary of scientific contributions to the field of icosahedral boron-rich solids, carried out in this thesis.

\subsection{Methodological development}

\section{Superatom special quasirandom structure method}

In first-principles studies, the general theoretical frameworks to address the problems of configurational disorder in crystalline solids are the cluster expansion procedure, the special quasirandom structure (SQS) method, as well as the coherent potential approximation (CPA). However, theoretical modeling of configurational disorder in icosahedral boron-rich solids, using either the cluster expansion procedure or the SQS method, is practically formidable, owing to their complex icosahedral structures composing of a number of inequivalent crystallographic sites. On the one hand, implementation of the CPA is not suitable for treating the configurational disorder in such materials due to a lack of possibility to consider lattice relaxations and uncertainties, related to charge transfer between alloy components in semiconductors. As for boron carbide, displaying a large solubility 
range of carbon, numerous kinds of $\mathrm{B} / \mathrm{C}$ substitutional defects are conceivable. It is demonstrated in Paper I that, in the dilute limit, it might not be necessary to take into account all kinds of defects in modeling configurational disorder in boron carbide, as many of them displaying high defect-formation energies might be neglected. As a result, configurational disorder in boron carbide may be modeled by focusing merely on low-energy $\mathrm{B} / \mathrm{C}$ substitutional defects. Also, in cases of icosahedral boron-rich solids, instead of considering each atom individually, one can view an icosahedral cluster, together with an interstitial unit, as a superatom. Combining these ideas together, configurational disorder in boron carbide, arising from a high concentration of low-energy B/C substitutional defect patterns, can thus be modeled by a random distribution of different superatoms, each defined based on low-energy defects. This is a foundation of the superatom special quasirandom structure (SA-SQS) method, in which the configuration of the superatoms is mimicking a random alloy pattern, according to the SQS method.

Configurational disorder in boron carbide, modeled at the superatomic level by the SA-SQS method, has been shown to provide a qualitatively good description in terms of the properties, as compared to the experiments. The long standing controversial issues, regarding the discrepancies in the properties of boron carbide between the experiments and previous theoretical calculations, have thus been resolved, strengthening the reliability of the method. The SA-SQS method, however, has some drawback, as discussed in Chapter 4 - for example, it can by chance lead to the formation of the unfavorable types of bonding for boron carbide, which could result in an unphysical prediction not only of the atomic configuration of disordered boron carbide, but also of the transition temperature to that particular disordered configuration. To study a change of configuration of boron carbide as a function of temperature, going beyond the SA-SQS level, the brute-force cluster expansion method in combination with Monte Carlo simulations is implemented. In this case, the atomic configurations of disordered boron carbide, modeled by the SA-SQS method, can be included as the input structures in the cluster expansion procedure in order to construct the configurational Hamiltonian, which is in turn utilized in the Monte Carlo simulations to extract the temperature-dependent effects of configurational disorder in boron carbide, as demonstrated in Paper VII.

Besides modeling configurational disorder in boron carbide, the SA-SQS method has been shown effective also in modeling mixing alloys between different icosahedral boron-rich solids, for instance $\left(\mathrm{B}_{6} \mathrm{O}\right)_{1-x}\left(\mathrm{~B}_{13} \mathrm{C}_{2}\right)_{x}$, and $\mathrm{B}_{12}\left(\mathrm{As}_{1-x} \mathrm{P}_{x}\right)_{2}$, whose mixing thermodynamics as well as properties can now be directly accessed through the standard density functional theory calculations.

\subsection{Materials stabilities and properties}

\section{Boron carbide}

In Paper I and II, it is demonstrated that configurational disorder of $\mathrm{B}$ and $\mathrm{C}$ atoms in boron carbide, especially the compositions of $\mathrm{B}_{4} \mathrm{C}$ and $\mathrm{B}_{13} \mathrm{C}_{2}$, thermodynamically favored at high temperature, has a significant impact on the materials 
properties. As for $\mathrm{B}_{4} \mathrm{C}$, its ground-state structural unit is given by $\mathrm{B}_{11} \mathrm{C}^{p}(\mathrm{CBC})$. At low temperature, the icosahedral carbon $\mathrm{C}^{p}$ atoms are well ordered by occupying the same polar sites for every icosahedron. Such arrangement of the $\mathrm{C}^{p}$ atoms has been shown from the theoretical point of view to results in a small distortion, reducing the crystal symmetry of $\mathrm{B}_{4} \mathrm{C}$ from the experimentally observed rhombohedral $(R \overline{3} m)$ to based-center monoclinic $(\mathrm{Cm})$. I have shown in Paper I that the higher rhombohedral symmetry of $\mathrm{B}_{4} \mathrm{C}$ is restored, induced by configurational disorder of the $\mathrm{C}^{p}$ atoms at the polar sites at elevated temperature. Two configurational order-disorder transitions are predicted for $\mathrm{B}_{4} \mathrm{C}$ at $870 \mathrm{~K}$ and $2325 \mathrm{~K}$, respectively. The configuration of $\mathrm{B}_{4} \mathrm{C}$ after the first transition can be described by random substitution of the $\mathrm{C}^{p}$ atoms only at the three polar sites, forming the top or bottom triangular face of the icosahedra, yielding the rhombohedral symmetry with the space group of $R 3 m$. The full rhombohedral symmetry $R \overline{3} m$ of $\mathrm{B}_{4} \mathrm{C}$, as observed in the experiments, is achieved after the second transition, describing by the coexistence of the random substitution of the $\mathrm{C}^{p}$ atoms at all six polar sites and the bipolar defects $\left(\mathrm{B}_{10} \mathrm{C}_{2}^{p}+\mathrm{B}_{12}\right)$. It is shown in Paper I and III that the intra- and inter-icosahedral $\mathrm{C}^{p}-\mathrm{C}^{p}$ bonds are unfavorable types of bonding for boron carbide. However, the implementation of the SA-SQS method to model the second disordered state of $\mathrm{B}_{4} \mathrm{C}$ with the space group of $R \overline{3} m$, where the $\mathrm{C}^{p}$ atoms configurationally disorder at all six polar sites, in Paper I can by chance lead to the formation of the unfavorable $\mathrm{C}^{p}-\mathrm{C}^{p}$ bonds, resulting in high-energy configurations of the disordered $\mathrm{B}_{4} \mathrm{C}$. Since such bonds can be avoided by short-range order with a minor reduction of entropy, the transition temperature from the $R 3 m$ to $R \overline{3} m$ phases of the disordered $\mathrm{B}_{4} \mathrm{C}$, predicted in Paper I, can be largely overestimated, as compared to the experimental situation.

The investigation of configurational evolution of $\mathrm{B}_{4} \mathrm{C}$ as a function of temperature in Paper VII, using a first-principles cluster expansion method, reveals that configurational disorder of $\mathrm{B}$ and $\mathrm{C}$ atoms in $\mathrm{B}_{4} \mathrm{C}$ essentially takes place within the icosahedra, whiles the triatomic inter-icosahedral chains are CBC and hardly change with temperature. It is shown that at $T \gtrsim 1200 \mathrm{~K}$, apart from the random substitution of the $\mathrm{C}^{p}$ atoms within the $\mathrm{B}_{11} \mathrm{C}^{p}$ icosahedra at all six polar sites and the formation of $\mathrm{B}_{10} \mathrm{C}_{2}^{p}$ and $\mathrm{B}_{12}$ icosahedra, readily taking place at lower temperature, a relatively small amount of the $\mathrm{C}^{p}$ atoms can also substitute for the equatorial boron $\mathrm{B}^{e}$ atoms, leading to formation of $\mathrm{B}_{11} \mathrm{C}^{e}(\mathrm{CBC})$ icosahedra. Despite being very unfavorable types of bonding for boron carbide, the intra-icosahedral $\mathrm{C}^{p}-\mathrm{C}^{p}$ bonds within the $\mathrm{B}_{10} \mathrm{C}_{2}^{p}$ icosahedra, the inter-icosahedral $\mathrm{C}^{p}-\mathrm{C}^{p}$ bonds, and the CCC chains, can also form at $T \gtrsim 1500 \mathrm{~K}$. They are, however, expected to form at very low concentration. These are in line with the study of dilute $\mathrm{B} / \mathrm{C}$ substitutional defects in $\mathrm{B}_{4} \mathrm{C}$, as shown in Paper $\mathrm{I}$, in which the energy costs to form those unfavorable bonds $(>1.0 \mathrm{eV})$ are relatively higher, as compared to the energy costs for the random substitution of icosahedral $\mathrm{C}$ atoms at the polar sites without forming the inter- and intra-icosahedral $\mathrm{C}^{p}-\mathrm{C}^{p}$ bonds as well as for the formation of $\mathrm{B}_{11} \mathrm{C}^{e}(\mathrm{CBC})$ icosahedra, ranging between $0.2 \mathrm{eV}$ and $0.8 \mathrm{eV}$.

As for $\mathrm{B}_{13} \mathrm{C}_{2}$, it is demonstrated in Paper II that the metallic low-temperature state of $\mathrm{B}_{13} \mathrm{C}_{2}$, represented by $\mathrm{B}_{12}(\mathrm{CBC})$, configurationally disorders at elevated 
temperature by which some of the $\mathrm{B}_{12}(\mathrm{CBC})$ units turn into the $\mathrm{B}_{11} \mathrm{C}^{e}(\mathrm{BBC})$ units without forming the $\mathrm{C}-\mathrm{C}$ bond between the icosahedra and the triatomic chains. This can be seen as formation of a pseudo-binary $\left[\mathrm{B}_{12}(\mathrm{CBC})\right]_{1-x}\left[\mathrm{~B}_{11} \mathrm{C}^{e}(\mathrm{BBC})\right]_{x}$ alloy, where the degree of configurational disorder $x$ increases with temperature. The existence of the $\mathrm{B}_{11} \mathrm{C}^{e}(\mathrm{BBC})$ units is found to partially or fully compensate the electron deficiency in the $\mathrm{B}_{12}(\mathrm{CBC})$ units, depending on their relative concentrations. As the temperature increases, the electron deficiency in $\mathrm{B}_{13} \mathrm{C}_{2}$, given by $\left[\mathrm{B}_{12}(\mathrm{CBC})\right]_{1-x}\left[\mathrm{~B}_{11} \mathrm{C}^{e}(\mathrm{BBC})\right]_{x}$, is gradually compensated. The metal-nonmetal transition in $\mathrm{B}_{13} \mathrm{C}_{2}$ is achieved at $2350 \mathrm{~K}$, where $x=0.5$, and is corresponding to the experimental observation that boron carbide is a semiconductor. The calculated electronic band gap of the disordered $\mathrm{B}_{13} \mathrm{C}_{2}$ is also in fairly good agreement with the experiments. Apart from the crystallographic and electronic properties, there also exists a large discrepancy in the elastic properties of boron carbide, particularly the composition of $\mathrm{B}_{13} \mathrm{C}_{2}$. In this case, the calculated elastic moduli of the low-temperature ordered state of $\mathrm{B}_{13} \mathrm{C}_{2}$ or $\mathrm{B}_{12}(\mathrm{CBC})$ are considerably lower, as compared to the experimental values. I have demonstrated in Paper IV that taking into account the influences of configurational disorder of $\mathrm{B}$ and $\mathrm{C}$ atoms, leading to the formation of the disordered $\mathrm{B}_{13} \mathrm{C}_{2}$ or $\left[\mathrm{B}_{12}(\mathrm{CBC})\right]_{0.5}\left[\mathrm{~B}_{11} \mathrm{C}^{e}(\mathrm{BBC})\right]_{0.5}$, can result in a drastic increase in the elastic moduli of the material, thus yielding an excellent agreement with the experiments.

For conclusion, I have demonstrated that configurational disorder of $\mathrm{B}$ and $\mathrm{C}$ atoms, predicted to be stable at elevated temperature, can explain the experimental observations of the materials properties, made at low temperature, thus resolving the discrepancies between the experiments and previous theoretical calculations, having been one of the controversial issues in the field of icosahedral boron-rich solids. One possible explanation is that the configurational disorder in boron carbide, thermodynamically favored at high temperature, might be frozen in, limited by insufficient atomic diffusion at low temperature due to strong interatomic covalent bonds, which are typical characteristic of icosahedral boron-rich solids. However, there is possibly also considerable quantitative overestimations of the order-disorder transition temperatures in some cases. This can be attributed to the approximations, used in the SA-SQS method to model configurational disorder of $\mathrm{B}$ and $\mathrm{C}$ atoms in boron carbide and the mean-field approach to estimated the transition temperatures.

In Paper III, the thermodynamic stability of carbon-rich boron carbide beyond the solubility limit of carbon, that is at. $\% \mathrm{C} \geqslant 20$, is investigated. I have demonstrated that, in addition to $\mathrm{B}_{4} \mathrm{C}, \mathrm{B}_{2.5} \mathrm{C}$ represented by $\mathrm{B}_{10} \mathrm{C}_{2}^{p}(\mathrm{CC})$ is predicted to be thermodynamically stable with respect to $\mathrm{B}_{4} \mathrm{C}$ and diamond under high pressure and also at elevated temperature. Depending on the temperature, the stability range of $\mathrm{B}_{2.5} \mathrm{C}$ is predicted to be within the range between 40 and $67 \mathrm{GPa}$. At $T \gtrsim 500 \mathrm{~K}$, the $\mathrm{C}^{p}$ atoms in $\mathrm{B}_{2.5} \mathrm{C}$ configurationally disorder at the polar sites, where they arrange themselves in such a way that the formation of a $\mathrm{C}^{p}-\mathrm{C}^{p}$ bond is avoid. On the one hand, $\mathrm{B}_{4} \mathrm{C}$ is predicted to undergo the phase decomposition at around $47 \mathrm{GPa}$ into $\gamma$-boron and $\mathrm{B}_{2.5} \mathrm{C}$, stable within a limited stability range before it subsequently decomposes into $\gamma$-boron and diamond upon increasing pressure. I have also demonstrated that $\mathrm{B}_{2.5} \mathrm{C}$ is both dynamically and 
mechanically stable at $0 \mathrm{GPa}$. One can thus expect that $\mathrm{B}_{2.5} \mathrm{C}$ will remain in a metastable state at ambient condition, due to a lack of atomic mobility if pressure is released at low temperature. To inspire attempts of experimental synthesis of $\mathrm{B}_{2.5} \mathrm{C}$, a possible synthesis route of $\mathrm{B}_{2.5} \mathrm{C}$ as well as a fingerprint for its characterization from the simulations of $\mathrm{x}$-ray powder diffraction pattern are provided in Paper III.

\section{Pseudo-binary $\left(\mathbf{B}_{6} \mathbf{O}\right)_{1-x}\left(\mathbf{B}_{13} \mathbf{C}_{2}\right)_{x}$ alloy}

Motivated by the recent experimental syntheses of composites of boron suboxide and boron carbide, I have investigated the mixing thermodynamics of a pseudobinary $\left(\mathrm{B}_{6} \mathrm{O}\right)_{1-x}\left(\mathrm{~B}_{13} \mathrm{C}_{2}\right)_{x}$ alloy. Similar to the modeling of configurational disorder in boron carbide, structural models of the alloy are obtained at the superatomic level, using the SA-SQS technique. The knowledge of configurational disorder, gained from the previous studies of boron carbide, is taken into account. By evaluating the stability of the alloys with respect to their constituent compounds, the pseudo-binary $T-x$ diagram of the $\left(\mathrm{B}_{6} \mathrm{O}\right)_{1-x}\left(\mathrm{~B}_{13} \mathrm{C}_{2}\right)_{x}$ alloy is outlined, as demonstrated in Paper IV. The phase diagram reveals a miscibility gap, existing at all temperatures up to the melting points of the materials. The gap demonstrates the coexistence of $\mathrm{B}_{6} \mathrm{O}$-rich and $\mathrm{B}_{13} \mathrm{C}_{2}$-rich domains in the $\left(\mathrm{B}_{6} \mathrm{O}\right)_{1-x}\left(\mathrm{~B}_{13} \mathrm{C}_{2}\right)_{x}$ alloy under equilibrium conditions, reflecting the character of composite materials. At low temperature, where $T \lesssim 1400 \mathrm{~K}$, the $\mathrm{B}_{6} \mathrm{O}$-rich and $\mathrm{B}_{13} \mathrm{C}_{2}$-rich regions are dominated by $\mathrm{B}_{12}(\mathrm{OO})$ and $\mathrm{B}_{12}(\mathrm{CBC})$, respectively. As the temperature increases, a part of the $\mathrm{C}$ atoms from the triatomic $\mathrm{CBC}$ chains can substitute for $\mathrm{B}^{e}$ atoms, residing in the icosahedra, in a similar fashion to the configurational disorder in $\mathrm{B}_{13} \mathrm{C}_{2}$, resulting in additional degree of configurational disorder in the $\left(\mathrm{B}_{6} \mathrm{O}\right)_{1-x}\left(\mathrm{~B}_{13} \mathrm{C}_{2}\right)_{x}$ alloy. Additionally, the intermixing of $\mathrm{B}_{6} \mathrm{O}$ and $\mathrm{B}_{13} \mathrm{C}_{2}$ to form solid solutions is predicted at high temperature, for example, a small solubility of $\sim 5 \% \mathrm{~B}_{6} \mathrm{O}$ in $\mathrm{B}_{13} \mathrm{C}_{2}$ and a solid solution of up to $\sim 20 \% \mathrm{~B}_{13} \mathrm{C}_{2}$ in $\mathrm{B}_{6} \mathrm{O}$ at $2000 \mathrm{~K}$. In this case, the mutual solubility of $\mathrm{B}_{6} \mathrm{O}$ and $\mathrm{B}_{13} \mathrm{C}_{2}$ should be taken as a lower limit due to the use of mean-field approximation, as described in Chapter 5. The study of the elastic properties of the $\left(\mathrm{B}_{6} \mathrm{O}\right)_{1-x}\left(\mathrm{~B}_{13} \mathrm{C}_{2}\right)_{x}$ alloys reveals a linear relationship between the elastic constants and the concentrations of the constituent compounds. Such a linear relation also holds for the elastic moduli of the alloys.

\section{Ternary boron-arsenic-phosphorus system}

In Paper V, the thermodynamic stability of compound and alloys in the ternary BAs-P system are examined. I have demonstrated that, in the binary B-As system, the icosahedral $\mathrm{B}_{12} \mathrm{As}_{2}$ is the only stable compound, whiles the zinc-blende BAs is thermodynamically unstable with respect to $\mathrm{B}_{12} \mathrm{As}_{2}$ and pure arsenic phase, given by gray arsenic, at $0 \mathrm{~K}$ and increasingly so at higher temperature, suggesting that BAs may merely exist as a metastable phase. This is in contrast to the binary B-P system, in which both icosahedral $\mathrm{B}_{12} \mathrm{P}_{2}$ and zinc-blende $\mathrm{BP}$ are predicted to be stable with respect to their pure phases, those are $\alpha$-rhombohedral boron, black phosphorus, and each other. As for the binary As-P system, $\mathrm{As}_{1-x} \mathrm{P}_{x}$ disordered 
alloys are predicted at elevated temperature - for example, a disordered solid solution of up to $\sim 75 \%$ As in black phosphorus as well as a small solubility of $\sim 1 \%$ $\mathrm{P}$ in gray arsenic at $T=750 \mathrm{~K}$, together with the presence of miscibility gaps. The calculated large solubility of As in black phosphorus is in line with the recent experimental syntheses of black phosphorus-typed $\mathrm{As}_{1-x} \mathrm{P}_{x}$ alloys with tunable compositions.

By evaluating the mixing thermodynamics of alloys between icosahedral $\mathrm{B}_{12} \mathrm{As}_{2}$ and $\mathrm{B}_{12} \mathrm{P}_{2}$, I have demonstrated a high tendency for intermixing of As and $\mathrm{P}$ atoms at the diatomic chain sites to form a $\mathrm{B}_{12}\left(\mathrm{As}_{1-x} \mathrm{P}_{x}\right)_{2}$ disordered alloy. The outlined $T-x$ phase diagram of the $\mathrm{B}_{12}\left(\mathrm{As}_{1-x} \mathrm{P}_{x}\right)_{2}$ alloy displays noticeable mutual solubility of the icosahedral subpnictides in each other even at room temperature as well as a closure of a pseudo-binary miscibility gap around $900 \mathrm{~K}$. Concerning a pseudo-binary $\mathrm{BAs}_{1-x} \mathrm{P}_{x}$ alloy, only a tiny amount of BAs is predicted to be able to dissolve in $\mathrm{BP}$ to form the $\mathrm{BAs}_{1-x} \mathrm{P}_{x}$ disordered alloy at elevated temperature. For example, less than $5 \% \mathrm{BAs}$ can dissolve in BP at $T=1000 \mathrm{~K}$. The small solubility limit of BAs in $\mathrm{BP}$ is attributed to the thermodynamic instability of BAs with respect to $\mathrm{B}_{12} \mathrm{As}_{2}$ and $\mathrm{As}$. As far as I am aware, neither $\mathrm{B}_{12}\left(\mathrm{As}_{1-x} \mathrm{P}_{x}\right)_{2}$ nor $\mathrm{BAs}_{1-x} \mathrm{P}_{x}$ alloy has been experimentally realized. I note also that the alloying $\mathrm{B}_{12} \mathrm{As}_{2}$ with $\mathrm{B}_{12} \mathrm{P}_{2}$ to form the $\mathrm{B}_{12}\left(\mathrm{As}_{1-x} \mathrm{P}_{x}\right)_{2}$ alloy is expected to lower thermal conductivity and thus possibly improve the thermoelectric figure of merit of the materials, as explained in Chapter 1.

\section{Boron subnitride}

Paper VI aims at clarifying the thermodynamic stability of three different compositions of boron subnitride, having been proposed so far in the literature. Those are, $\mathrm{B}_{6} \mathrm{~N}$ represented by $\mathrm{B}_{12}(\mathrm{~N}-\mathrm{N}), \mathrm{B}_{13} \mathrm{~N}_{2}$ represented by $\mathrm{B}_{12}(\mathrm{NBN})$, and $\mathrm{B}_{38} \mathrm{~N}_{6}$ represented by $\left[\mathrm{B}_{12}(\mathrm{~N}-\mathrm{N})\right]_{0.33}\left[\mathrm{~B}_{12}(\mathrm{NBN})\right]_{0.67}$. I have shown that, out of these subnitrides, only $\mathrm{B}_{38} \mathrm{~N}_{6}$, given by $\left[\mathrm{B}_{12}(\mathrm{~N}-\mathrm{N})\right]_{0.33}\left[\mathrm{~B}_{12}(\mathrm{NBN})\right]_{0.67}$, is thermodynamically stable from $0 \mathrm{GPa}$ up to $7.5 \mathrm{GPa}$, depending on the temperature. Beyond this pressure, also $\mathrm{B}_{38} \mathrm{~N}_{6}$ becomes unstable, and decompose into cubic boron nitride and $\alpha$-tetragonal boron-like boron subnitride $\mathrm{B}_{50} \mathrm{~N}_{2}$. I have also demonstrated that $\left[\mathrm{B}_{12}(\mathrm{~N}-\mathrm{N})\right]_{0.33}\left[\mathrm{~B}_{12}(\mathrm{NBN})\right]_{0.67}$ is dynamically and mechanically stable, and is an electrical semiconductor.

By comparing the calculated lattice parameters and simulated x-ray powderdiffraction pattern of $\left[\mathrm{B}_{12}(\mathrm{~N}-\mathrm{N})\right]_{0.33}\left[\mathrm{~B}_{12}(\mathrm{NBN})\right]_{0.67}$ to the experimental data, existing in the literature, I have suggested that instead of being represented solely either by $\mathrm{B}_{12}(\mathrm{NBN})$ or by $\mathrm{B}_{12}(\mathrm{~N}-\mathrm{N})$ as often claimed previously, the atomic configuration of the as-synthesized boron subnitrides should be described by the mixture of $\mathrm{B}_{12}(\mathrm{NBN})$ and $\mathrm{B}_{12}(\mathrm{~N}-\mathrm{N})$ with a ratio of $2: 1$, corresponding to the composition of $\mathrm{B}_{38} \mathrm{~N}_{6}$. I thus propose that $\mathrm{B}_{38} \mathrm{~N}_{6}$ is a stable composition of boron subnitride. 


\section{CHAPTER 8}

\section{Outlook}

My studies in Paper I, II, and IV demonstrate that configurational disorder both in $\mathrm{B}_{4} \mathrm{C}$ and $\mathrm{B}_{13} \mathrm{C}_{2}$, arising from a high concentration of low-energy $\mathrm{B} / \mathrm{C}$ substitutional defects, can account for several discrepancies between experiments and previous theoretical calculations in terms of the material properties. The issue regarding the overestimation of the electronic band gap of $\mathrm{B}_{4} \mathrm{C}$ obtained from the DFT calculations, however, has not been fully resolved yet (Paper I). It is worth noting that, generally, electronic band gaps of semiconducting materials tend to decrease upon increasing the temperature, while the temperature in the standard DFT calculations is at absolute zero. Thus, apart from the influences of the configurational disorder, one may also try to investigate the temperature-dependent effects of the electronic band gap of $\mathrm{B}_{4} \mathrm{C}$ by performing ab initio molecular dynamics (AIMD) simulations.

In general, a methodological first step to account for the influence of lattice dynamics on the stabilities of materials is to implement either the harmonic or the quasi-harmonic approximation (see Chapter 3). However, one should be aware that, within these approximations, the potential energy surface of atoms in a crystal is extracted from their equilibrium positions at $T=0 \mathrm{~K}$. At very high temperature, the two approximations can become inaccurate and thus fail to describe the thermal properties of the crystal [169]. This is attributed to strong anharmonic effects, originating from which the atoms strongly vibrate and largely displaced from their equilibrium positions. To include the effects of anharmonicity in determining the stabilities of materials at high temperature, one needs to go beyond the harmonic and quasi-harmonic approximations, by implementing more sophisticated methods, based on AIMD - for example, temperature dependent effective potential (TDEP) $[170,171]$ or methods, relying on the formally exact thermodynamic integration approach [172]. This is motivated by the thermodynamic stability of configurationally disordered $\mathrm{B}_{4} \mathrm{C}$ (Paper I) and $\mathrm{B}_{13} \mathrm{C}_{2}$ (Paper 
II) at very high temperature, where the effects of anharmonicity are expected to be distinctively strong.

As it is not trivial to use conventional diffraction techniques to quantify the presence of configurational disorder in boron carbide due to the similar characteristics of boron and carbon atoms, one may try calculating extended x-ray absorption fine structure (EXAFS) and core level shift (CLS) spectra for both ordered and disordered boron carbides, using first-principles methods, and use them as fingerprints to trace the presence of configurational disorder in boron carbide samples by comparing with experimentally measured spectra, focusing on $\mathrm{C}(1 \mathrm{~s})$ and $\mathrm{B}(1 \mathrm{~s})$ edges.

The study in Paper III suggests a new phase of boron carbide with the composition of $\mathrm{B}_{2.5} \mathrm{C}$, stable under high pressure. The $\mathrm{B}_{2.5} \mathrm{C}$ phase is also expected to remain in a metastable state at ambient condition, as indicated by its dynamical and mechanical stabilities at zero pressure. One should thus try synthesizing the $\mathrm{B}_{2.5} \mathrm{C}$ phase in experiment and investigate its properties. According to the predictions, carried out in the thesis, the $\mathrm{B}_{2.5} \mathrm{C}$ phase is a wide-bandgap semiconductor and is expected to be a superhard material, even harder than $\mathrm{B}_{4} \mathrm{C}$. Besides the $\mathrm{B}_{2.5} \mathrm{C}$ phase, it is also of interest to try synthesizing the $\mathrm{B}_{12}\left(\mathrm{As}_{1-x} \mathrm{P}_{x}\right)_{2}$ disordered alloys and investigating their properties. This is motivated not only by the predictions of a noticeable mutual solubility of $\mathrm{B}_{12} \mathrm{As}_{2}$ and $\mathrm{B}_{12} \mathrm{P}_{2}$ in each other even at room temperature, but also by a tendency of improving the thermoelectric properties of boron subpnictides due to a large mass difference between As and $\mathrm{P}$ atoms, giving high alloy phonon scattering (Paper V).

In Paper VII, I have demonstrated the possibility to investigate the change in the atomic configuration of $\mathrm{B}_{4} \mathrm{C}$ with temperature beyond the mean-field approximation and SA-SQS level by using the first-principles cluster expansion method in combination with Monte Carlo simulations. It is thus interesting to implement such a method in order to study configurational evolution of boron carbide also at other compositions within the single-phase region, apart from $\mathrm{B}_{4} \mathrm{C}$.

Recently, ${ }^{10} \mathrm{~B}$-rich $\mathrm{B}_{4} \mathrm{C}$ thin films, as synthesized by physical or chemical vapor depositions, have been realized as a new generation of large area neutron detector [35]. Since the temperature for depositing the material is limited by the melting temperature of the substrate, in particular aluminium, as-deposited $\mathrm{B}_{4} \mathrm{C}$ films are practically amorphous $\left(\mathrm{a}-\mathrm{B}_{4} \mathrm{C}\right)$. It is also of interest to investigate atomic structure of the $\mathrm{a}-\mathrm{B}_{4} \mathrm{C}$ thin films, which has so far been rarely addressed in the literature [80]. Indeed, determination of atomic structure of amorphous materials is a challenge for both theoreticians and experimentalists due to the lack of long-range crystal order. Such a challenge may possibly be overcome by using a combined theoretical and experimental approach. As a first step, one extracts the structural information - for example, pair distribution function (PDF) - from a set of structural models of $\mathrm{a}-\mathrm{B}_{4} \mathrm{C}$, generated using the quantum molecular dynamics (QMD) or stochastic quenching (SQ) methods. The theoretical PDFs are then compared with the experimental PDFs, obtained from neutron scattering and synchrotron $\mathrm{x}$-ray diffraction measurements of the $\mathrm{a}-\mathrm{B}_{4} \mathrm{C}$ films, in order to draw information on the atomic structure of $\mathrm{a}_{-} \mathrm{B}_{4} \mathrm{C}$ from the candidate models. 


\section{Bibliography}

[1] T. L. Aselage et al. "Anomalous Seebeck Coefficient in Boron Carbides". In: MRS Proceedings 97 (1987), p. 27.

[2] B. Morosin et al. "Rhombohedral crystal structure of compunds containing boron-rich icosahedra". In: AIP Conference Proceedings 140 (1986), pp. 7086.

[3] H. K. Clark and J. L. Hoard. "The Crystal Structure of Boron Carbide". In: Journal of American Chemical Society 65 (1943), p. 2115.

[4] H. L. Yakel. "The Crystal Structure of a Boron-Rich Boron Carbide". In: Acta Crystallographica Section B 31 (1975), p. 1797.

[5] B. Morosin et al. "Neutron powder diffraction refinement of boron carbides. Nature of intericosahedral chains". In: Journal of Alloys and Compounds 226 (1995), p. 121.

[6] D. Emin. "Unusual properties of icosahedral boron-rich solids". In: Journal of Solid State Chemistry 179 (2006), pp. 2791-2798.

[7] H. Bolmgren, T. Lundström, and S. Okada. "Structure refinement of the boron suboxide $\mathrm{B}_{6} \mathrm{O}$ by the Rietveld method". In: AIP Conference Proceedings 231 (1991), p. 197.

[8] M. Kobayashi et al. "Structure of $\mathrm{B}_{6} \mathrm{O}$ boron-suboxide by Rietveld refinement". In: Journal of Materials Sceince 28 (1993), p. 2129.

[9] K. Shirai. "Electronic structures and mechanical properties of boron and boron-rich crystals (Part I)". In: Journal of Superhard Materials 32.3 (2010), pp. 205-225.

[10] L. Wang et al. "Spectroscopic properties of crystalline elemental boron and the implications on $B_{11}$ C-CBC". In: Royal Society of Chemistry 3 (2013), pp. 25374-25387.

[11] J. L. Hoard et al. "The structure analysis of $\beta$-rhombohedral boron". In: Journal of Solid State Chemistry 1 (1970), pp. 268-277. 
[12] M. J. Setten et al. "Thermodynamic Stability of Boron: The Role of Defects and Zero Point Motion". In: Journal of the American Chemical Society 129.9 (2007), pp. 2458-2465.

[13] D. W. Bullett. "Structure and bonding in crystalline boron and $\mathrm{B}_{12} \mathrm{C}_{3}$ ". In: Journal of Physics C: Solid State Physics 15.3 (1982), pp. 415-426.

[14] A. R. Oganov et al. "Ionic high-pressure form of elemental boron". In: Nature 457 (2009), pp. 863-867.

[15] E. Yu. Zarechnaya et al. "Superhard Semiconducting Optically Transparent High Pressure Phase of Boron". In: Physical Review Letters 102 (2009), p. 185501.

[16] M. Vlasse et al. "The Crystal Structure of $\mathrm{SiB}_{6}$ ". In: Journal of Solid State Chemistry 63 (1986), pp. 31-45.

[17] D. Emin. "Icosahedral boron-rich solids". In: Physics Today 40 (1987), p. 55.

[18] H. C. Longuet-Higgins and M. de V. Roberts. "The electronic structure of an icosahedron of boron atoms". In: Proceedings of the Royal Society London, Series A 230 (1955), p. 110.

[19] H. Zhang, S. Yao, and M. Widom. "Predicted phase diagram of boroncarbon-nitrogen". In: Physical Review B 93 (2016), p. 144107.

[20] F. Thévenot. "Boron Carbide - A Comprehensive Review". In: Journal of the European Ceramic Society 6 (1990), pp. 205-225.

[21] J. E. Zorzi, C. A. Perottoni, and J. A. H. da Jornada. "Hardness and wear resistance of $\mathrm{B}_{4} \mathrm{C}$ ceramics prepared with several additives". In: Materials Letters 59 (2005), pp. 2932-2935.

[22] M. Chen, J. W. McCauley, and K. J. Hemker. "Shock-Induced Localized Amorphization in Boron Carbide". In: Science 299.5612 (2003), pp. 15631566 .

[23] R. Freer. "The Physics and Chemistry of Carbides, Nitrides and Borides". In: Kluwer Academic Publishers, The Netherlands (1990), p. 113.

[24] D. He et al. "Boron suboxide: As hard as cubic boron nitride". In: Applied Physics Letters 81.4 (2002), p. 643.

[25] M. Herrmann et al. "Boron suboxide ultrahard materials". In: International Journal of Refractory Metals and Hard Materials 39 (2013), p. 53.

[26] D. Emin. "Icosahedral Boron-Rich Solids as Refractory Semiconductors". In: MRS Proceedings 97 (1987), p. 3.

[27] Y. Gong et al. "Demonstration of boron arsenide heterojunctions: A radiation hard wide band gap semiconductor device". In: Applied Physics Letters 96 (2010), p. 223506.

[28] H. Werheit. "Boron-rich solids: a chance for high-efficiency high-temperature thermoelectric energy conversion". In: Materials Science and Engineering B 29 (1995), p. 228. 
[29] Y. Gong et al. "Thermal conductivity and Seeback coefficient of icosahedral boron arsenide films on silicon carbide". In: Journal of Applied Physics 108 (2010), p. 084960.

[30] C. D. Frye et al. "Seebeck coefficient and electrical resistivity of single crystal $\mathrm{B}_{12} \mathrm{As}_{2}$ at high temperatures". In: Journal of the Physical Society of Japan 82 (2013), p. 095001.

[31] G. W. Hollenberg and G. Walther. "The Elastic Modulus and Fracture of Boron Carbide". In: Journal of the American Ceramic Society 63 (1980), pp. 610-613.

[32] D. Simeone et al. "Study of boron carbide evolution under neutron irradiation by Raman spectroscopy". In: Journal of Nuclear Materials 277 (2000), pp. $1-10$.

[33] D. Emin and T. L. Aselage. "A proposed boron-carbide-based solid-state neutron detector". In: Journal of Applied Physics 97 (2005), p. 013529.

[34] J. L. Lacy et al. "Boron-coated straw as a replacement for ${ }^{3} \mathrm{He}-$ based neutron detectors". In: Physics Research Section A: Accelerators, Spectrometers, Detectors and Associated Equipment 652 (2011), pp. 359-363.

[35] C. Höglund et al. " $\mathrm{B}_{4} \mathrm{C}$ thin films for neutron detection". In: Journal of Applied Physics 111 (2012), p. 104908.

[36] V. Domnich et al. "Boron Carbide: Structure, Properties, and Stability under Stress". In: Journal of the American Ceramic Society 94.11 (2011), p. 3605 .

[37] I. Jiménez et al. "Photoemission and x-ray absorption study of boron carbide and its surface thermal stability". In: Physical Review B 57 (1998), p. 13167.

[38] J. M. Sanchez, F. Ducastelle, and D. Gratias. "Generalized cluster description of multicomponent systems". In: Physica A: Statistical Mechanics and its Applications 128 (1984), pp. 334-350.

[39] J. M. Sanchez. "Cluster expansions and the configurational energy of alloys". In: Physical Review B 48 (1993), 14013(R).

[40] J. M. Sanchez. "Cluster expansions and the configurational theory of alloys". In: Physical Review B 81 (2010), p. 224202.

[41] A. Zunger et al. "Special Quasirandom Structures". In: Physical Review Letters 65.3 (1990), p. 353.

[42] E. I. Isaev et al. "Impact of lattice vibrations on equation of state of the hardest boron phase". In: Physical Review B 83 (2011), p. 132106.

[43] M. Bouchacourt and F. Thévenot. "ÉTUDES SUR LE CARBURE DE BORE. III. DOMAINE D'EXISTENCE DE LA PHASE CARBURE DE BORE". In: Journal of the Less-Common Metals 59 (1978), pp. 139-152.

[44] M. Beauvy. "Stoichiometric Limits of Carbon-Rich Boron Carbide Phase". In: Journal of the Less-Common Metals 90 (1983), pp. 169-175. 
[45] H. Okamoto. "B-C (boron-carbon)". In: Journal of Phase Equilibria 13 (1992), p. 436.

[46] W. P. Huhn and M. Widom. "A Free Energy Model of Boron Carbide". In: Journal of Statistical Physics 150 (2013), p. 432.

[47] V. F. Sears. "Neutron scattering lengths and cross sections". In: Neutron News 3.3 (1992), pp. 26-37.

[48] A. H. Silver and P. J. Bray. "Nuclear Magnetic Resonance Study of Boron Carbide". In: The Journal of Chemical Physics 31 (1959), p. 247.

[49] M. N. Alexander. "Nuclear magnetic resonance studies of the structure of boron carbides". In: AIP Conference Proceedings 140 (1986), p. 168.

[50] D. Lee, P. J. Bray, and T. L. Aselage. "The NQR and NMR studies of icosahedral borides". In: Journal of Physics: Condensed Matter 11.22 (1999), p. 4435.

[51] A. C. Larson. "Comments concerning the crytal structure of $\mathrm{B}_{4} \mathrm{C}$ ". In: AIP Conference Proceedings 140 (1986), p. 109.

[52] G. H. Kwei and B. Morosin. "Structures of the Boron-Rich Boron Carbides from Neutron Powder Diffraction: Implications for the Nature of the InterIcosahedral Chains". In: The Journal of Physical Chemistry 100.19 (1996), p. 8031.

[53] D. R. Armstrong et al. "The nature of the chemical bonding in boron carbide. IV. Electronic band structure of boron carbide, $\mathrm{B}_{13} \mathrm{C}_{2}$, and the model of the structure $\mathrm{B}_{12} \mathrm{C}_{3}$ ". In: Acta Crystallographica Section B 39 (1983), pp. 324-329.

[54] G. Fanchini, J. W. McCauley, and M. Chhowalla. "Behavior of Disordered Boron Carbide under Stress". In: Physical Review Letters 97 (2006), p. 035502.

[55] S. Aydin and M. Simsek. "Hypothetically superhard boron carbide structures with a $\mathrm{B}_{11} \mathrm{C}$ icosahedron and three-atom chain". In: Physica Status Solidi B 246.1 (2009), p. 62.

[56] R. Lazzari et al. "Atomic Structure and Vibrational Properties of Icosahedral B 4 C Boron Carbide". In: Physical Review Letters 83 (1999), p. 3230.

[57] M. Widom and W. P. Huhn. "Prediction of orientational phase transition in boron carbide". In: Solid State Sciences 14 (2012), p. 1648.

[58] F. Mauri, N. Vast, and C. J. Pickard. "Atomic Structure of Icosahedral $\mathrm{B}_{4} \mathrm{C}$ Boron Carbide from a First Principles Analysis of NMR Spectra". In: Physical Review Letters 87 (2001), p. 085506.

[59] D. M. Bylander and L. Kleinman. "Structure of $\mathrm{B}_{13} \mathrm{C}_{2}$ ". In: Physical Review B 42.2 (1991), p. 1487.

[60] J. E. Saal, S. Shang, and Z. K. Liu. "The structural evolution of boron carbide via an initio calculations". In: Applied Physics Letters 91 (2007), p. 231915 . 
[61] N. Vast, J. Sjakste, and E. Betranhandy. "Boron carbides from first principles". In: Journal of Physics: Conference Series 176 (2009), p. 012002.

[62] T. L. Aselage and R. G. Tissot. "Lattice constants of boron carbides". In: Journal of the American Ceramic Society 75.8 (1992), p. 2207.

[63] D. R. Tallant et al. "Boron carbide structure by Raman spectoscopy". In: Physical Review B 40.8 (1989), p. 5649.

[64] T. L. Aselage, D. R. Tallant, and D. Emin. "Isotope dependencies of Raman spectra of $\mathrm{B}_{12} \mathrm{As}_{2}, \mathrm{~B}_{12} \mathrm{P}_{2}, \mathrm{~B}_{12} \mathrm{O}_{2}$, and $\mathrm{B}_{12+x} \mathrm{C}_{3-x}$ : Bonding of intericosahedral chains". In: Physical Review B 56.6 (1997), p. 3122.

[65] T. L. Aselage and D. R. Tallant. "Association of broad icosahedral Raman bands with substitutional disorder in $\mathrm{SiB}_{3}$ and boron carbide". In: Physical Review B 57.5 (1998), p. 2675.

[66] C. Wood and D. Emin. "Conduction mechanism in boron carbide". In: Physical Review B 29 (1984), p. 4582.

[67] L. Zuppiroli, N. Papandreou, and R. Kormann. "The dielectric response of boron carbide due to hopping conduction". In: Journal of Applied Physics 70 (1991), p. 246.

[68] H. Werheit. "On excitons and other gap states in boron carbide". In: Journal of Physics: Condensed Matter 18 (2006), p. 10655.

[69] H. Werheit, H. Binnenbruck, and A. Hausen. "Optical properties of boron carbide and comparison with $\beta$-rhombohedral boron". In: Physica Status Solidi B 47 (1971), p. 153.

[70] H. Werheit, M. Laux, and U. Kuhlmann. "Optical Interband Transitions of Boron Carbide". In: Physica Status Solidi B 172 (1992), K81.

[71] H. Werheit et al. "Interband Critical Points of Some Icosahedral Boron-Rich Solids". In: Journal of Solid State Chemistry 133 (1997), pp. 132-139.

[72] M. Calandra, N. Vast, and F. Mauri. "Superconductivity from doping boron icosahedra". In: Physical Review B 69 (2004), p. 224505.

[73] D. M. Bylander, L. Kleinman, and S. Lee. "Self-consistent calcualtions of the energy bands and bonding properties of $\mathrm{B}_{12} \mathrm{C}_{3}$ ". In: Physical Review $B$ 47 (1990), p. 10056.

[74] V. I. Ivashchenko, V. I. Shevchenko, and P. E. A. Turchi. "First-Principles study of the atomic and electronic structures of crystalline and amorphous B 4 C". In: Physical Review B 80 (2009), p. 235208.

[75] H. Dekura, K. Shirai, and A. Yanase. "Metallicity of boron carbides at high pressure". In: Journal of Physics: Conference Series 215 (2010), p. 012117.

[76] M. M. Balakrishnarajan, P. D. Pancharatna, and R. Hoffmann. "Structure and bonding in boron carbide: The invicibility of imperfections". In: New Journal of Chemistry 31 (2007), p. 473.

[77] R. Schmechel and H. Werheit. "Structural defects of some icosahedral boronrich solids and their correlation with the electronic properties". In: Journal of Solid State Chemistry 154 (2000), p. 61. 
[78] B. L. Gyorffy. "Coherent-Potential Approximation for a NonoverlappingMuffin-Tin-Potential Model of Random Substitutional Alloys". In: Physical Review B 5.6 (1972), p. 2382.

[79] J. K. Sonber et al. "Synthesis, Densification and Characterization of Boron Carbide". In: Transactions - Indian Ceramic Society 72.2 (2013), p. 100.

[80] C. Pallier et al. "Structure of an Amorphous Boron Carbide Film: An Experimental and Computational Approach". In: Chemistry of Materials 25 (2013), p. 2618.

[81] S. V. Konovalikhin and V. I. Ponomarev. "Carbon in Boron Carbide: The Crystal Structure of $\mathrm{B}_{11.4} \mathrm{C}_{3.6}$ ". In: Russian Journal of Inorganic Chemistry 54 (2009), p. 197.

[82] A. Jay et al. "Carbon-rich icosahedral boron carbide designed from first principles". In: Applied Physics Letters 105 (2014), p. 031914.

[83] A. Jay. "Conception in silico d'une nouvelle phase de carbure de bore". $\mathrm{PhD}$ thesis. Ecole Doctorale Polytechnique, 2015.

[84] A. Hushur et al. "High-pressure phase transition makes $\mathrm{B}_{4.3} \mathrm{C}$ boron carbide a wide-gap semiconductor". In: Journal of Physics: Condensed Matter 28 (2016), p. 045403.

[85] T. Fujii et al. "X-ray diffraction study of $\mathrm{B}_{4} \mathrm{C}$ under high pressure". In: Journal of Physics: Conference Series 215 (2010), p. 012011.

[86] X. Q. Yan et al. "Depressurization Amorphization of Single-Crystal Boron Carbide". In: Physical Review Letters 102 (2009), p. 075505.

[87] I. Solodkyi et al. "Synthesis of $\mathrm{B}_{6} \mathrm{O}$ powder and spark plasma sintering of $\mathrm{B}_{6} \mathrm{O}$ and $\mathrm{B}_{6} \mathrm{O}-\mathrm{B}_{4} \mathrm{C}$ ceramics". In: Journal of the Ceramic Society of Japan 121.11 (2013), p. 950.

[88] H. Itoh, I. Maekawa, and H. Iwahara. "High Pressure Sintering of $\mathrm{B}_{6} \mathrm{O}$ Powder and Properties of Sintered Compact". In: JOURNAL-SOCIETY OF MATERIAL SCIENCE JAPAN 47 (1998), p. 1000.

[89] H. Itoh, I. Maekawa, and H. Iwahara. "Microstructure and mechanical properties of $\mathrm{B}_{6} \mathrm{O}-\mathrm{B}_{4} \mathrm{C}$ sintered composites prepared under high pressure". In: Journal of Material Sceince 35 (2000), p. 693.

[90] C. Chen et al. "B ${ }_{6}$ O-Based Composite to Rival Polycrystalline Cubic Boron Nitride". In: Advanced Materials 19 (2007), p. 4288.

[91] M. Carrard, D. Emin, and L. Zuppiroli. "Defect clustering and self-healing of electron-irradiated boron-rich solids". In: Physical Review B 51.17 (1995), p. 11270.

[92] S. P. Huber et al. "Self-healing in $\mathrm{B}_{12} \mathrm{P}_{2}$ through Mediated Defect Recombination". In: Chemistry of Materials 28 (2016), p. 8415.

[93] T.L. Aselage and D. Emin. Beta cell device using icosahedral boride compounds. 2002. 
[94] S. Bakalova et al. "Energy band structure and optical response function of icosahedral $\mathrm{B}_{12} \mathrm{As}_{2}$ : A spectroscopic ellipsometry and first-principles calculational study". In: Physical Review B 81 (2010), p. 075114.

[95] J. Wu et al. "High pressure X-ray diffraction study on icosahedral boron arsenide $\left(\mathrm{B}_{12} \mathrm{As}_{2}\right)$ ". In: Journal of Physics and Chemistry of Solids 72 (2011), p. 144 .

[96] C. E. Whiteley et al. "Semiconducting icosahedral boron arsenide crystal growth for neutron detection". In: Journal of Crystal Growth 318 (2011), p. 553 .

[97] V. A. Mukhanov et al. "Self-propagating high-temperature synthesis of boron subphosphide $\mathrm{B}_{12} \mathrm{P}_{2}$ ". In: Journal of Superhard Materials 36.1 (2014), p. 18.

[98] V. A. Mukhanov et al. "Ultra-fast mechanochemical synthesis of boron phosphide, BP and $\mathrm{B}_{12} \mathrm{P}_{2}$ ". In: Dalton Transactions 45 (2016), p. 10122.

[99] J. A. Perri, S. L. Placa, and B. Post. "New group III-group V compounds: BP and BAs". In: Acta Crystallographica 11 (1958), p. 310.

[100] F. V. Williams and R. A. Ruehrwein. "The Preparation and Properties of Boron Phosphides and Arsenides" ". In: Journal of the American Chemical Society 82.6 (1960), p. 1330.

[101] T. L. Chu and A. E. Hyslop. "Crystal Growth and Properties of Boron Monoarsenide". In: Journal of Applied Physics 43.276 (1972).

[102] L. Lindsey, D. A. Broido, and T. L. Reinecke. "First-Principles Determination of Ultrahigh Thermal Conductivity of Boron Arsenide: A Competitor for Diamond?" In: Physical Review Letters 111 (2013), p. 025901.

[103] B. Lv et al. "Experimental study of the proposed super-thermal-conductor: BAs". In: Applied Physics Letters 106 (2015), p. 074105.

[104] J. Kim et al. "Thermal and thermoelectric transport measurements of an individual boron arsenide microstructure". In: Applied Physics Letters 108 (2016), p. 201905.

[105] N. H. Protik et al. "Ab initio study of the effect of vacancies on the thermal conductivity of boron arsenide". In: Physical Review B 94 (2016), p. 045207.

[106] H. Dumont and Y. Monteil. "Some aspects on thermodynamic properties, phase diagram and alloy formation in the ternary system BAs-GaAsPart I: Analysis of BAs thermodynamic properties". In: Journal of Crystal Growth 290 (2006), p. 410.

[107] S. Drablia et al. "First principles calculations of structural, electronic, thermodynamic and optical properties of $\mathrm{BAs}_{1-x} \mathrm{P}_{x}$ alloy". In: Physica Scripta 79 (2009), p. 045002.

[108] S. V. Ovsyannikov et al. "Raman spectroscopy of $\mathrm{B}_{12} \mathrm{As}_{2}$ and $\mathrm{B}_{12} \mathrm{P}_{2}$ up to 120 GPa: Evidence for structural distortion". In: Physical Review B 81 (2010), 140103(R). 
[109] S. Adenwalla et al. "Boron carbide/n-silicon carbide heterojunction diodes". In: Applied Physics Letters 79.26 (2001), p. 4357.

[110] D. Emin and T. L. Aselage. "A proposed boron-carbide-based solid-state neutron detector". In: Journal of Applied Physics 97 (2005), p. 013529.

[111] O. O. Kurakevych and V. L. Solozhenko. "Experimental Study and Critical Review of Structural, Thermodynamic and Mechanical Properties of Superhard Refractory Boron Suboxide ${ }_{6}$ O". In: Journal of Superhard Materials 33.6 (2011), p. 421.

[112] G. A. Slack and K. E. Morgan. "Some crystallography, chemistry, physics, and thermodynamics of $\mathrm{B}_{12} \mathrm{O}_{2}, \mathrm{~B}_{12} \mathrm{P}_{2}, \mathrm{~B}_{12} \mathrm{As}_{2}$, and related alpha-boron type crystals". In: Journal of Physics and Chemistry of Solids 75 (2014), p. 1054.

[113] G. A. Slack and K. E. Morgan. "Crystallography, semiconductivity, thermoelectricity, and other properties of boron and its compounds, especially $\mathrm{B}_{6}$ O". In: Solid State Sciences 47 (2015), p. 43.

[114] C. E. Whiteley, M. J. Kirkham, and J. H. Edgar. "The coefficients of thermal expansion of boron arsenide $\left(\mathrm{B}_{12} \mathrm{As}_{2}\right)$ between $25^{\circ} \mathrm{C}$ and $850{ }^{\circ} \mathrm{C}$ ". In: Journal of Physics and Chemistry of Solids 74 (2013), p. 673.

[115] J. B. Condon et al. "The Kinetics of the Boron Plus Nitrogen Reaction". In: Inorganic Chemistry 15.9 (1976), p. 2173.

[116] H. Saitoh, K. Yoshida, and W. A. Yarbrough. "Crystal structure of new composition boron-rich boron nitride using Raman spectroscopy". In: Journal of Materials Research 8.1 (1993), p. 8.

[117] H. Hubert et al. "High-Pressure, High-Temperature Syntheses in the B-CN-O System". In: Journal of Solid State Chemistry 133 (1997), p. 356.

[118] V. L. Solozhenko and O. O. Kurakevych. "New boron subnitride $\mathrm{B}_{13} \mathrm{~N}_{2}$ : HP-HT synthesis, structure and equation of state". In: Journal of Physics: Conference Series 121 (2008), p. 062001.

[119] V. L. Solozhenko and O. O. Kurakevych. "Chemical interaction in the BBN system at high pressures and temperatures. Synthesis of novel boron subnitrides". In: Journal of Solid State Chemistry 182 (2009), p. 1359.

[120] V. L. Solozhenko et al. "Phase Diagram of the B-BN System at 5 GPa". In: Journal of Physical Chemistry B 114 (2010), p. 5819.

[121] M. Born and R. Oppenheimer. "Zur Quantentheorie der Molekeln". In: Annalen der Physik 389 (1927), pp. 714-733.

[122] F. Bloch. "Über die quantenmechanic der elektronen in kristallgittern". In: Zeitschrift für Physik 52 (1928), p. 555.

[123] L. H. Thomas. "The calculation of atomic fields". In: Mathematical Proceedings of the Cambridge Philosophical Society 23.05 (1927), pp. 542548.

[124] E. Fermi. "Un metodo statistico per la determinazione di alcune priorieta dell'atome". In: Accad. Naz. Lincei 71 (1927), pp. 1253-1266. 
[125] P. Hohenberg and W. Kohn. "Inhomogeneous electron gas". In: Physical Review 136.3B (1964), B864-B871.

[126] R. M. Martin. "Electronic structure, basic theory and pratical methods". In: Cambridge University Press (2004).

[127] W. Kohn and L. J. Sham. "Self-consistent equations including exchange and correlation effects". In: Physical Review 140.4A (1965), A1133-A1138.

[128] D. M. Ceperley and B. J. Alder. "Ground state of the electron gas by a stochastic method". In: Physical Review Letters 45 (1980), pp. 566-569.

[129] O. Gunnarsson, M. Jonson, and B. I. Lundqvist. "Descriptions of exchange and correlation effects in inhomogeneous electron systems". In: Physical Review B 20 (1979), p. 3136.

[130] A. D. Becke. "Density functional exchange-energy approximation with correct asymtotic behavior". In: Physical Review A 38 (1988), p. 3098.

[131] Y. Wang and J. P. Perdew. "Correlation hole of the spin-polarized electron gas, with exact small-wave-vector and highdensity scaling". In: Physical Review B 44 (1991), p. 13298.

[132] J. Perdew, K. Burke, and M. Ernzerhof. "Generalized Gradient Approximation Made Simple". In: Physical Review Letters 77 (1996), p. 3865.

[133] J. P. Perdew et al. "Density-Functional Theory for Fractional Particle Number: Derivative Discontinuities of the Energy". In: Physical Review Letters 49 (1982), p. 1691.

[134] J. P. Perdew and M. Levy. "Physical Content of the Exact Kohn-Sham Orbital Energies: Band Gaps and Derivative Discontinuities". In: Physical Review Letters 51 (1983), p. 1884.

[135] A. D. Becke. "Density-functional thermochemistry. III. The role of exact exchange". In: The Journal of Chemical Physics 98 (1993), p. 5648.

[136] C. Lee, W. Yang, and R. G. Parr. "Development of the Colle-Salvetti correlation-energy formula into a functional of the electron density". In: Physical Review B 37 (1988), p. 785.

[137] C. Adamo and V. Barone. "Toward reliable density functional methods without adjustable parameters: The PBE0 model". In: The Journal of Chemical Physics 110 (1999), p. 6158.

[138] J. Heyd, G. E. Scuseria, and M. Ernzerhof. "Hybrid functionals based on a screened Coulomb potential". In: The Journal of Chemical Physics 118 (2003), p. 8207.

[139] J. Heyd and G. E. Scuseria. "Efficient hybrid density functional calculations in solids: Assessment of the Heyd-Scuseria-Ernzerhof screened Coulomb hybrid functional". In: The Journal of Chemical Physics 121 (2004), p. 1187.

[140] J. Heyd, G. E. Scuseria, and M. Ernzerhof. "Erratum: "Hybrid functionals based on a screened Coulomb potential" [J. Chem. Phys. 118, 8207 (2003)]". In: The Journal of Chemical Physics 124 (2006), p. 219906. 
[141] A. D. Becke and E. R. Johnson. "A simple effective potential for exchange". In: The Journal of Chemical Physics 124 (2006), p. 221101.

[142] F. Tran and P. Blaha. "Accurate band gaps of semiconductors and isulators with a semilocal exchange-correlations potential". In: Physical Review Letters 102 (2009), p. 226401.

[143] H. Hellmann. "A new approximation method in the problem of many electrons". In: The Journal of Chemical Physics 3.1 (1935), p. 61.

[144] H. Hellmann and W. Kassatotschkin. "Metallic binding according to the combined approximation procedure". In: The Journal of Chemical Physics 4.5 (1936), p. 324.

[145] D. R. Hamann, M. Schlüter, and C. Chiang. "Norm-Conserving Pseudopotentials". In: Physical Review Letters 43 (1979), p. 1494.

[146] D. Vanderbilt. "Soft self-consistent pseudopotentials in a generalized eigenvalue formalism". In: Physical Review B 41 (1990), 7892(R).

[147] P. E. Blöchl. "Projector augmented-wave method". In: Physical Review B 50.24 (1994), p. 17953.

[148] M. Born and T. von Kármán. "Uber Schwingungen in Raumgittern". In: Physikalische Zeitschrift 13 (1912), p. 297.

[149] A. A. Maradudin and S. H. Vosko. "Symmetry properties of the normal vibrations of a crystal". In: Reviews of Modern Physics 40 (1968), p. 1.

[150] A. A. Maradudin and A. E. Fein. "Scattering of neutrons by an anharmonic crystal". In: Physical Review 128 (1962), p. 2589.

[151] P. Brüesch. Phonons: Theory and Experiments I. Springer Berlin Heidelberg, 1982.

[152] G. Leibfried and W. Ludwig. "Theory of anharmonic effects in crystals". In: Solid State Physics 12 (1961), p. 275.

[153] J. W. D. Connolly and A. R. Williams. "Density-functional theory applied to phase transformations in transition-metal alloys". In: Physical Review B 27 (1983), 5169(R).

[154] P. Soven. "Coherent-Potential Model of Substitutional Disordered Alloys". In: Physical Review 156 (1967), p. 809.

[155] A. V. Ruban and I. A. Abrikosov. "Configurational thermodynamics of alloys from first principles: effective cluster interactions". In: Reports on Progress in Physics 71 (2008), p. 046501.

[156] H. Shin et al. "Cohesion energetic of carbon allotropes: Quantum Monte Carlo study". In: The Journal of Chemical Physics 140 (2014), p. 114702.

[157] S. Yao, W. P. Huhn, and M. Widom. "Phase transitions of boron carbide: Pair interaction model of high carbon limit". In: Solid State Sciences 47 (2015), p. 21. 
[158] B. Alling. "Metal to semiconductor transition and phase stability of $\mathrm{Ti}_{1-x} \mathrm{Mg}_{x} \mathrm{~N}_{y}$ alloys investigated by first-principles calculations". In: Physical Review B 89 (2014), p. 085112.

[159] D. A. Porter, K. E. Easterling, and M. Sherif. Phase Transformations in Metals and Alloys. UK: CRC press, 2009.

[160] M. Dahlqvist, B. Alling, and J. Rosén. "Stability trends of MAX phases from first principles". In: Physical Review B 81.220102(R) (2010).

[161] F. Tasnádi, M. Odén, and I. A. Abrikosov. "Ab initio elastic tensor of cubic $\mathrm{Ti}_{0.5} \mathrm{Al}_{0.5} \mathrm{~N}$ alloys: Dependence of elastic constants on size and shape of the supercell model and their convergence". In: Physical Review B 85 (2012), p. 144112.

[162] R. Golesorkhtabar et al. "ElaStic: A tool for calculating second-order elastic constants from first principles". In: Computer Physics Communications 184 (2013), p. 1861.

[163] J. F. Nye. Physical Properties of Crystals: Their Representation by Tensors and Matrices. USA: Oxford University Press, 1985.

[164] L. Vitos. Computational Quantum Mechanics for Materials Engineers: The EMTO Method and Applications (Engineering Materials and Processes). Berlin: Spinger, 2010.

[165] S. Q. Wang and H. Q. Ye. "Ab initio elastic constants for the lonsdaleite phases of C, Si and Ge". In: Journal of Physics: Condensed Matter 15 (2003), p. 5307.

[166] M. Moakher and A. N. Norris. "The Closest Elastic Tensor of Arbitrary Symmetry to an Elasticity Tensor of Lower Symmetry". In: Journal of Elasticity 85 (2006), p. 215.

[167] F. Mouhat and F.-X. Coudert. "Necessary and Sufficient Elastic Stability Conditions in Various Crystal Systems". In: Physical Review B 90 (2014), p. 224104.

[168] G. Simmons and H. Wang. Single Crystal Elastic Constants and Calculated Aggregate Properties: A Handbook. Cambridge: M.I.T Press, 1971.

[169] O. Hellman, I. A. Abrikosov, and S. I. Simak. "Lattice dynamics of anharmonic solids from first principles". In: Physical Review B 84 (2011), p. 180301.

[170] O. Hellman et al. "Temperature dependent effective potential method for accurate free energy calculation of solids". In: Physical Review B 87 (2013), p. 104111.

[171] O. Hellman and I. A. Abrikosov. "Temperature-dependent effective thirdorder interatomic force constants from first principles". In: Physical Review B 88 (2013), p. 144301.

[172] A. l. Duff et al. "Improved method of calculating ab initio high-temperature thermodynamic properties with application to ZrC". In: Physical Review B 91 (2015), p. 214311. 
$\underline{72}$

BIBLIOGRAPHY 
[I] First-principles study of configurational disorder in $B_{4} C$ using a superatom-special quasirandom structure method.

A. Ektarawong, S. I. Simak, L. Hultman, J. Birch, and B. Alling.

Physical Review B, 90, 024204 (2014).

[II] Configurational order-disorder induced metal-nonmetal transition in $\mathbf{B}_{13} \mathbf{C}_{2}$ studied with first-principles superatom-special quasirandom structure method.

A. Ektarawong, S. I. Simak, L. Hultman, J. Birch, and B. Alling.

Physical Review B, 92, 014202 (2015).

[III] Carbon-rich icosahedral boron carbides beyond $\mathbf{B}_{4} \mathbf{C}$ and their thermodynamic stabilities at high temperature and pressure from first principles.

A. Ektarawong, S. I. Simak, and B. Alling.

Physical Review B, 94, 054104 (2016).

[IV] Effects of configurational disorder on the elastic properties of icosahedral boron-rich alloys based on $\mathrm{B}_{6} \mathrm{O}, \mathrm{B}_{13} \mathrm{C}_{2}$, and $\mathrm{B}_{4} \mathrm{C}$, and their mixing thermodynamics.

A. Ektarawong, S. I. Simak, L. Hultman, J. Birch, F. Tasnádi, F. Wang and B. Alling.

The Journal of Chemical Physics, 144, 134503 (2016). 
[V] First principles predictions of stabilities and instabilities of compounds and alloys in the ternary B-As-P system.

A. Ektarawong, S. I. Simak, and B. Alling.

Submitted to Physical Review B (2017).

[VI] Thermodynamic stability and properties of boron subnitrides from first principles.

A. Ektarawong, S. I. Simak, and B. Alling.

Physical Review B, 95, 064206 (2017).

[VII] Configurational evolution with temperature of all crystallographic sublattices in $\mathbf{B}_{4} \mathbf{C}$ from first-principles cluster expansion.

A. Ektarawong, S. B. Maisel, S. I. Simak, B. Grabowski, J. Neugebauer, and B. Alling.

In manuscript (2017). 
Related, not included publication

Control of $\mathrm{Ti}_{1-x} \mathrm{Si}_{x} \mathrm{~N}$ nanostructure via tunable metal-ion momentum transfer during HIPIMS/DCMS co-deposition.

G. Greczynski, J. Patscheider, J. Lu, B. Alling, A. Ektarawong, J. Jensen, I. Petrov, J. E. Greene, and L. Hultman.

Surface and Coatings Technology, 280, 174 (2015). 


\section{CHAPTER 9}

\section{Summary of included publications}

\section{Paper I}

First-principles study of configurational disorder in $\mathrm{B}_{4} \mathrm{C}$ using a superatom-special quasirandom structure method.

\section{Summary}

This paper highlights the development of a methodological framework, namely a superatom-special quasirandom structure (SA-SQS) method, for modeling configurational disorder in boron carbide, arising from a high concentration of low-energy $\mathrm{B} / \mathrm{C}$ substitutional defects. To illustrate its practical implementation, the developed method is applied in a first-principles study of $\mathrm{B}_{4} \mathrm{C}$, given by $\mathrm{B}_{11} \mathrm{C}^{p}(\mathrm{CBC})$. It is shown from thermodynamic consideration that the $\mathrm{C}^{p}$ atoms, which are well ordered by occupying the same polar sites for every icosahedron at low temperature, configurationally disorder at elevated temperature. The configurational disorder of the $\mathrm{C}^{p}$ atoms is found to remove the small monoclinic distortion, existing in the low-temperature ordered phase of $\mathrm{B}_{4} \mathrm{C}$, and thus the higher rhombohedral symmetry of $\mathrm{B}_{4} \mathrm{C}$, as observed in experiments, can be restored.

\section{Author's contribution}

I took part in planning of the project, performed all the calculations, participated in the evaluation and interpretation of the results, and was responsible for writing the manuscript.

\section{Paper II}

Configurational order-disorder induced metal-nonmetal transition in $\mathrm{B}_{13} \mathrm{C}_{2}$ studied with first-principles superatom-special quasirandom structure method. 


\section{Summary}

This paper demonstrates first-principles investigation of configurational disorder in $\mathrm{B}_{13} \mathrm{C}_{2}$. At zero Kelvin, the atomic configuration of $\mathrm{B}_{13} \mathrm{C}_{2}$ is presumably represented by $\mathrm{B}_{12}(\mathrm{CBC})$. Due to its electron deficiency, $\mathrm{B}_{12}(\mathrm{CBC})$ is metallic. As the temperature increases, $\mathrm{B}_{13} \mathrm{C}_{2}$ configurationally disorders and undergoes a metalto-nonmetal transition by which some of $\mathrm{B}_{12}(\mathrm{CBC})$ turn into $\mathrm{B}_{11} \mathrm{C}^{e}(\mathrm{BBC})$. The presence of $\mathrm{B}_{11} \mathrm{C}^{e}(\mathrm{BBC})$ splits off some valence states into the band gap that in turn gradually compensates the electron deficiency in $\mathrm{B}_{12}(\mathrm{CBC})$, thus resulting in a semiconducting character of $\mathrm{B}_{13} \mathrm{C}_{2}$ at high temperature, and in line with experimental observation that boron carbide is a semiconductor.

\section{Author's contribution}

I took part in planning of the project, performed all the calculations, participated in the evaluation and interpretation of the results, and was responsible for writing the manuscript.

\section{Paper III}

Carbon-rich icosahedral boron carbides beyond $\mathrm{B}_{4} \mathrm{C}$ and their thermodynamic stabilities at high temperature and pressure from first principles.

\section{Summary}

In this paper, the thermodynamic stability of carbon-rich boron carbides, in which at.\% $\mathrm{C} \geqslant 20$, is investigated using first-principles calculations. It is demonstrated that, apart from $\mathrm{B}_{4} \mathrm{C}$ generally addressed in the literature, $\mathrm{B}_{2.5} \mathrm{C}$ represented by $\mathrm{B}_{10} \mathrm{C}_{2}^{p}(\mathrm{CC})$ is predicted to be thermodynamically stable under high pressure, ranging between 40 and $67 \mathrm{GPa}$, with respect to $\mathrm{B}_{4} \mathrm{C}$ as well as pure boron and carbon phases. $\mathrm{B}_{2.5} \mathrm{C}$ is also expected to be metastable at ambient pressure, as indicated by its dynamical and mechanical stabilities at $0 \mathrm{GPa}$. A possible route for experimental synthesis of $\mathrm{B}_{2.5} \mathrm{C}$ as well as a fingerprint for its characterization from the simulations of $\mathrm{x}$-ray powder diffraction pattern are provided.

\section{Author's contribution}

I planned the project, did all the calculations, took a major part in the evaluation and interpretation of the results, and was responsible for writing the manuscript.

\section{Paper IV}

Effects of configurational disorder on the elastic properties of icosahedral boronrich alloys based on $\mathrm{B}_{6} \mathrm{O}, \mathrm{B}_{13} \mathrm{C}_{2}$, and $\mathrm{B}_{4} \mathrm{C}$, and their mixing thermodynamics.

\section{Summary}

This paper consists of two parts. The first part highlights the influences of configurational disorder on the elastic properties of boron carbide. The results indicate that, when configurational disorder of boron and carbon atoms are taken into account, an excellent agreement on elastic moduli of between theory and experiment 
is achieved for boron carbide, especially $\mathrm{B}_{13} \mathrm{C}_{2}$. The second part focuses on the mixing thermodynamics between $\mathrm{B}_{6} \mathrm{O}$ and $\mathrm{B}_{13} \mathrm{C}_{2}$. The pseudo-binary $T-x$ phase diagram of the $\left(\mathrm{B}_{6} \mathrm{O}\right)_{1-x}\left(\mathrm{~B}_{13} \mathrm{C}_{2}\right)_{x}$ alloy reveals the coexistence of $\mathrm{B}_{6} \mathrm{O}$-rich and $\mathrm{B}_{13} \mathrm{C}_{2}$-rich domains, as indicated by the miscibility gap, existing at all temperature up to the melting point of the materials. The intermixing of $\mathrm{B}_{6} \mathrm{O}$ and $\mathrm{B}_{13} \mathrm{C}_{2}$ to form solid solutions is also predicted at high temperature. Upon increasing the temperature, a part of the $\mathrm{C}$ atoms from the triatomic $\mathrm{CBC}$ chains can substitute for $\mathrm{B}^{e}$ atoms, residing in the icosahedra, resulting in an additional degree of configurational disorder in the $\left(\mathrm{B}_{6} \mathrm{O}\right)_{1-x}\left(\mathrm{~B}_{13} \mathrm{C}_{2}\right)_{x}$ alloy.

\section{Author's contribution}

I took part in planning of the project, performed all the calculations, participated in the evaluation and interpretation of the results, and was responsible for writing the manuscript.

\section{Paper V}

First principles predictions of stabilities and instabilities of compounds and alloys in the ternary B-As-P system.

\section{Summary}

The thermodynamic stability of compounds and alloys in the ternary B-As-P system is examined using first-principles method. It is shown that zinc-blende BP and icosahedral $\mathrm{B}_{12} \mathrm{P}_{2}$ are both stable compounds in the binary B-P system, while in the case of B-As system zinc-blende BAs is found to be thermodynamically unstable with respect to icosahedral $\mathrm{B}_{12} \mathrm{As}_{2}$ and pure arsenic phase at 0 Kelvin and increasingly so at higher temperature, indicating that BAs may merely exist as a metastable phase. For the As- $\mathrm{P}$ system, $\mathrm{As}_{1-x} \mathrm{P}_{x}$ disordered alloys are predicted at elevated temperature. Concerning the ternary B-As-P system, the intermixing of As and $\mathrm{P}$ atoms at the diatomic chain sites of the icosahedral phase to form $\mathrm{B}_{12}\left(\mathrm{As}_{1-x} \mathrm{P}_{x}\right)_{2}$ alloys is predicted even at room temperature. As for the zincblende phase, only a tiny amount of BAs is predicted to be able to dissolve in $\mathrm{BP}$ to form $\mathrm{BAs}_{1-x} \mathrm{P}_{x}$ disordered alloys at elevated temperature, which is attributed to the thermodynamic instability of BAs with respect to $\mathrm{B}_{12} \mathrm{As}_{2}$ and As.

\section{Author's contribution}

I planned the project, did all the calculations, took a major part in the evaluation and interpretation of the results, and was responsible for writing the manuscript.

\section{Paper VI}

Thermodynamic stability and properties of boron subnitrides from first principles.

\section{Summary}

This paper demonstrates a first-principles study of the thermodynamic stability of three different compositions of $\alpha$-rhombohedral boron-like boron subnitride, hav- 
ing been proposed so far in the literature. Those are, $\mathrm{B}_{6} \mathrm{~N}, \mathrm{~B}_{13} \mathrm{~N}_{2}$, and $\mathrm{B}_{38} \mathrm{~N}_{6}$, represented respectively by $\mathrm{B}_{12}(\mathrm{~N}-\mathrm{N}), \mathrm{B}_{12}(\mathrm{NBN})$, and $\left[\mathrm{B}_{12}(\mathrm{~N}-\mathrm{N})\right]_{0.33}\left[\mathrm{~B}_{12}(\mathrm{NBN})\right]_{0.67}$. It is shown that, out of these subnitrides, only $\mathrm{B}_{38} \mathrm{~N}_{6}$ is thermodynamically stable from $0 \mathrm{GPa}$ up to $7.5 \mathrm{GPa}$, depending on the temperature. Beyond this pressure, also $\mathrm{B}_{38} \mathrm{~N}_{6}$ becomes unstable, and decomposes into cubic boron nitride and $\alpha$ tetragonal boron-like boron subnitride $\mathrm{B}_{50} \mathrm{~N}_{2}$. The calculated lattice parameters and simulated x-ray powder-diffraction pattern of $\left[\mathrm{B}_{12}(\mathrm{~N}-\mathrm{N})\right]_{0.33}\left[\mathrm{~B}_{12}(\mathrm{NBN})\right]_{0.67}$ are in good agreement with the experimental data of boron subnitride, existing in the literature, thus suggesting that $\mathrm{B}_{38} \mathrm{~N}_{6}$ is a stable composition of $\alpha$ rhombohedral boron-like boron subnitride.

\section{Author's contribution}

I planned the project, did all the calculations, took a major part in the evaluation and interpretation of the results, and was responsible for writing the manuscript.

\section{Paper VII}

Configurational evolution with temperature of all crystallographic sublattices in $\mathrm{B}_{4} \mathrm{C}$ from first-principles cluster expansion.

\section{Summary}

In this paper, the evolution of the atomic configuration of $\mathrm{B}_{4} \mathrm{C}$ as a function of temperature is studied using a first-principles cluster expansion in combination with Monte Carlo simulations. It is demonstrated that configurational disorder of $\mathrm{B}$ and $\mathrm{C}$ atoms essentially takes place within the icosahedra, resulting mainly in $\mathrm{B}_{11} \mathrm{C}^{p}$, and also in $\mathrm{B}_{12}, \mathrm{~B}_{10} \mathrm{C}_{2}^{p}$, and $\mathrm{B}_{11} \mathrm{C}^{e}$. The triatomic inter-icosahedral chains, on the other hand, are $\mathrm{CBC}$ and hardly change with temperature. Despite being unfavorable types of bonding for boron carbide, low concentrations of the interand intra-icosahedral $\mathrm{C}^{p}-\mathrm{C}^{p}$ bonds as well as triatomic $\mathrm{CCC}$ chains can also form at very high temperature.

\section{Author's contribution}

I carried out all of the density functional theory calculations, took part in performing Monte Carlo simulations, contributed in the interpretation of the results, and was responsible for writing the manuscript. 


\section{Papers}

The articles associated with this thesis have been removed for copyright reasons. For more details about these see:

http://urn.kb.se/resolve? urn:nbn:se:liu:diva-136813 